\title{
REAL-WORLD EVALUATION OF DYNAMIC ECO-DRIVING CONNECTED VEHICLE TECHNOLOGY ON AN ARTERIAL ROADWAY WITH SEMI- ACTUATED SIGNALS \\ By
}

Brooke Jones

A thesis submitted to the Faculty of Graduate and Postdoctoral Affairs in partial fulfillment of the requirements for the degree of

Master of Applied Science

In

Civil Engineering

Carleton University

Ottawa, Ontario

(C) 2018

Brooke Jones 


\begin{abstract}
Numerous strategies are being explored to reduce fuel consumption and the amount of greenhouse gas emissions produced by the transportation sector. One of these strategies revolves around optimizing driving behaviour, especially in the vicinity of signalized intersections. Real-world testing of EcoDrive, a connected vehicle application, was conducted over a period of months on an arterial roadway with semi-actuated and connected traffic signals. Green light optimal speed advisories and velocity planning messages were communicated by means of two-way wireless network connectivity to drivers based on the current vehicle position and real-time signal phase information. Vehicle positioning, at a single second resolution, was used to estimate emissions by way of a state of the art emission modeling software. A reduction in carbon dioxide equivalent emissions of $9.62 \%\left(\mathrm{CI}_{95} 1.74\right.$ to $\left.1.02 \mathrm{~kg} / \mathrm{h}\right)$ and a $9.64 \%\left(\mathrm{CI}_{95} 0.65\right.$ to $\left.0.38 \mathrm{~L} / \mathrm{h}\right)$ fuel savings were observed while travel time was not significantly altered.
\end{abstract}




\section{Acknowledgements}

I would like to express my deepest gratitude to my supervisor, Dr. Ata Khan. Your guidance and encouragement has been greatly appreciated throughout my academic career, but especially in the creation of this dissertation.

I am grateful to my colleagues at Carleton University, particularly for the dedication of their time and participation in the days of testing.

I would like to thank The City of Ottawa Project Manager, Greg Kent and his team for providing an amazing opportunity for me though my inclusion in this pilot project, and for their provision of additional traffic network information.

I am thankful for the team at Traffic Technology Services, Inc. who provided excellent support for the EcoDrive application.

Finally, this thesis would not have been possible without my family and friends who have provided me with endless support. 


\section{Table of Contents}

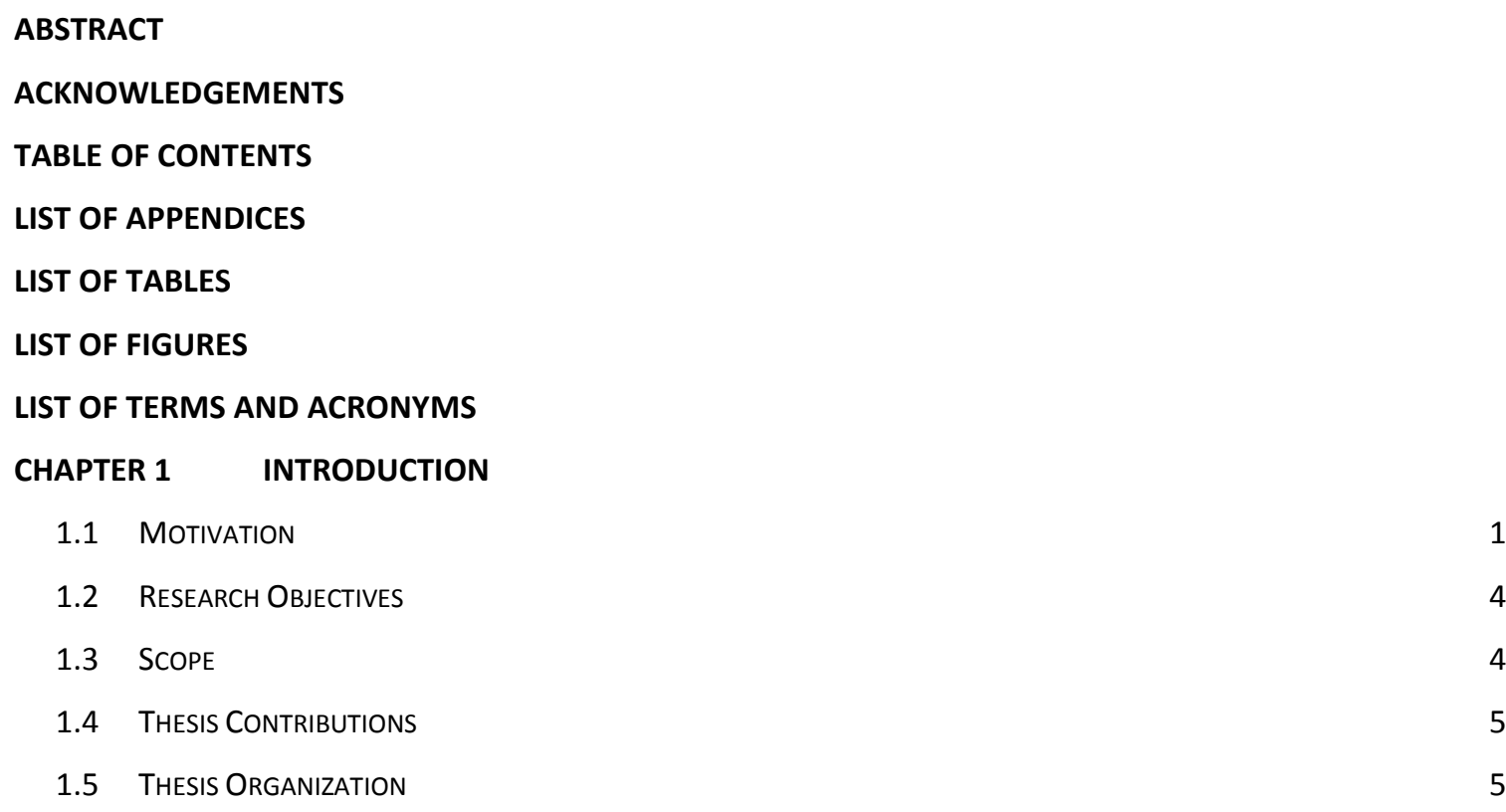

CHAPTER 2 BACKGROUND INFORMATION

7

2.1 TrafFic Network Optimization at Signalized Intersections 7

2.2 ECO-DRIVING CONCEPT 11

2.3 Connected Vehicles 13

2.4 Communication TeChnologies for Connected Vehicle Applications 14

2.5 Connected Vehicle Technology for Traffic Management 15

$\begin{array}{llr}\text { CHAPTER } 3 & 18\end{array}$

$\begin{array}{lll}3.1 & \text { DRIVING SIMULATOR STUDIES } & 18\end{array}$

3.1.1 Traffic Light Assistance System (TLAS) 18

3.2 Microscopic TRAfFic SimUlations 20

3.2.1 Green Wave 21

3.2.2 Connected Vehicle Dynamic Reservation Systems 22

3.2.3 Velocity Planning 23

3.2.4 Field Trials 32

3.3 REAL-WORLD TESTING $\quad 40$

3.3.1 GLOSA In Vehicle Traffic Signal Advisory System (ITSAS) 41

$\begin{array}{lll}\text { CHAPTER } 4 & \text { CORRIDOR DEFINITION AND STUDY DESCRIPTION }\end{array}$

4.1 The Hunt Club Road Corridor (HCRC)

$\begin{array}{lll}4.2 & \text { Trafaic Signals } & 47\end{array}$

$\begin{array}{llr}4.3 & \text { SPEED LIMIT } & 49\end{array}$

iv 
4.4 SAMPLE SIZE REQUIREMENT

CHAPTER 5 ECODRIVE SYSTEM ARCHITECTURE

$\begin{array}{llr}5.1 & \text { ECODRIVE FRONTENd APPLICATION } & 54\end{array}$

$\begin{array}{lll}5.2 & \text { The INSTRUMENTEd CARLETON VAN } & 57\end{array}$

CHAPTER 6 DATA ACQUISITION AND FORMATTING

$\begin{array}{llr}6.1 & \text { DATA ACQUISITION } & 58\end{array}$

$\begin{array}{llr}6.2 & \text { Arcmap EVAluation } & 61\end{array}$

6.3 Cartesian Coordinate Based Calculations 64

$\begin{array}{llr}6.4 & \text { STOPS } & 65\end{array}$

6.5 INITIAL DATA SCREENING $\quad 66$

6.5.1 EcoDrive Malfunction $\quad 66$

$\begin{array}{lll}\text { 6.5.2 Accident } & 66\end{array}$

$\begin{array}{lll}\text { 6.5.3 Lunch Hour } & 67\end{array}$

$\begin{array}{llr}\text { 6.5.4 Heavy Traffic Events } & 67\end{array}$

CHAPTER $7 \quad$ EMISSIONS SIMULATION METHODOLOGY 70

7.1 Motor Vehicle Emissions Simulation SOftware (MOVES) 70

$\begin{array}{lll}7.2 & \text { MOVES ANALYSIS } & 72\end{array}$

$\begin{array}{ll}\text { 7.2.1 MOVES Run Setup } & 72\end{array}$

$\begin{array}{llr}7.2 .2 & \text { RunSpec } & 73\end{array}$

$\begin{array}{lll}\text { 7.2.3 Project Data Manager } & 77\end{array}$

CHAPTER 8 EVALUATION OF ECODRIVE TECHNOLOGY 81

$\begin{array}{llr}8.1 & \text { Final OUtLIER ANALYSIS } & 81\end{array}$

8.2 Significance Testing Of the CoRe Variables 83

8.2.1 Travel Time Analysis $\quad 84$

8.2.2 Fuel Consumption and Emissions Analysis 85

$\begin{array}{lll}\text { 8.2.3 Secondary Factors } & 87\end{array}$

$\begin{array}{lll}8.3 & \text { TRAJECTORY COMPARISON } & 92\end{array}$

$\begin{array}{lll}8.4 & \text { EVAluation OF RESUlts } & 94\end{array}$

$\begin{array}{ll}\text { 8.4.1 Network Configuration } & 96\end{array}$

$\begin{array}{lll}\text { 8.4.2 Queve Length } & 99\end{array}$

CHAPTER 9 SUMMARY AND CONCLUSIONS

$\begin{array}{lll}\text { CHAPTER } 10 & \text { REFERENCES } & 106\end{array}$ 


\section{List of Appendices}

$\begin{array}{lll}\text { APPENDIX A } & \text { DATA FORMATTING EQUATIONS } & 114\end{array}$

$\begin{array}{lll}\text { APPENDIX B } & \text { TRIP RESULTS } & 116\end{array}$

$\begin{array}{lll}\text { APPENDIX C } & \text { ADDITIONAL MAPS } & 122\end{array}$

$\begin{array}{lll}\text { APPENDIX D } & \text { EQUIPPED VAN } & 128\end{array}$

$\begin{array}{lll}\text { APPENDIXE } & \text { R SCRIPTS } & 131\end{array}$

$\begin{array}{llr}\text { APPENDIX F } & \text { R OUTPUTS } & 146\end{array}$

\section{List of Tables}

Table 1: Mean week day traffic volumes along the corridor during the study

Table 2: Test of differences results for Average speed, GHG emissions and fuel consumption 


\section{List of Figures}

Figure 1 GHG emissions from the transportation sector in Canada from 1990 to 2015 [1] 3

Figure 2: Five components to fuel efficient driving techniques taught in Canada [14] 12

Figure 3: Fuel savings as a function of communication ranges [38] 26

Figure 4: Eco-Approach and Departure human machine interface [7] 35

Figure 5: Intersection locations on the Hunt Club Road Corridor 45

Figure 6: High-level EcoDrive system architecture [53] 53

Figure 7: IVU display message options 56

Figure 8: IVU display as seen during testing 57

Figure 9: UTM zones within Canada and the shape of a UTM zone [57] 62

Figure 10: GIS Test run starting and termination points 64

Figure 11: Outlier point identification $\quad 68$

Figure 12: The Municipality of Ottawa (top) and St Lawrence County (bottom) maps 75

Figure 13: MOVES Operating Mode bins for light duty vehicles [67] 78

Figure 14: Histogram of bootstrap difference in fuel consumption and its confidence interval 86

Figure 15: Histogram of bootstrap difference in $\mathrm{CO}_{2} \mathrm{E}$ and its confidence interval 87

Figure 16: Comparison of uninformed and informed driver trajectories 94

Figure 17: Sample second-by-second analysis a 116

Figure 18: Sample second-by-second analysis b 117

$\begin{array}{ll}\text { Figure 19: Sample second-by-second analysis c } & 118\end{array}$

Figure 20: Initial trip result visualization 119

Figure 21: Initial trip visualization at times of day 119

Figure 22: Summary results with Average speeds and MOVES outputs a 120

Figure 23: Summary results with Average speeds and MOVES outputs b 121

Figure 26: ArcMap GPS slow points evaluated for unusual traffic conditions 122

Figure 27: Cycling facilities on the Hunt Club Road Corridor [74] 123

Figure 28: Pedestrian facilities on the Hunt Club Road Corridor [74] 124

Figure 29: Ottawa urban trucking routes [75] 125

Figure 30: City of Ottawa rural trucking routes [76] 126

Figure 31: OC Transpo bus routes along the Hunt Club Road Corridor [77] 127

Figure 32: Carleton University test vehicle (the Van) 128

Figure 33: Configuration of equipment inside the Van 129

Figure 34: GPS antenna attached to the roof of the Van [78] 130

Figure 35: All recorded System OFF summary statistics 146

vii 
Figure 36: All recorded IVU active summary statistics

Figure 37: All recorded IVU providing countdowns only summary statistics 147

Figure 38: All recorded IVU providing EcoDrive recommendation summary statistics 147

Figure 39: All informed driver runs without replacement 148

Figure 40: Uninformed driver final summary a 148

Figure 41: Uninformed driver final summary b 149

Figure 42: Uninformed driver final summary c 149

Figure 43: Uninformed driver final summary d 150

Figure 44: Informed driver final summary a 150

Figure 45: Informed driver final summary b 151

Figure 46: Informed driver final summary c 151

Figure 47: Informed driver final summary d 152

Figure 48: Frequency distributions of variables for uninformed and informed driver phases 153

Figure 49: Trip outlier representation through boxplots 154

Figure 50: Frequency distributions of core variables for uninformed and informed driver phases 155

Figure 51: Frequency distributions of secondary variables for uninformed and informed driver phases 156 


\section{List of Terms and Acronyms}

$\mathrm{CI}_{95}$ - Confidence Interval

GHG - Green house gas

$\mathrm{CO}_{2}$ - Carbon Dioxide

IVU - In-Vehicle Unit

SPaT - Signal phase and timing

MOVES - MOtor Vehicle Emissions Simulator software

SCOOT - Split Cycle and Offset Optimization Technique

COSMOS - Cycle, Offset, Split MOdel for Seoul

V2X - two-way wireless communication vehicle to: vehicle, infrastructure or device

DSRC - Dedicated Short Range Connectivity

MAP - packets containing information on the physical geometry of intersections

EcoDrive - the application developed for the study

TLAS - Traffic Light Assistance System

ASL - Advisory Speed Limit

GLOSA - Green Light Optimal Speed Advisory

CMEM - Comprehensive Modal Emission Model

VSP - Vehicle Specific Power

AERIS - Application for the Environment: Real-time Information Synthesis

TFHRC - Turner Fairbank Highway Research Centre

ITSAS - In Vehicle Traffic Signal Advisory System

OBD - On Board Device

HCRC - Hunt Club Road Corridor

CTL - Central Limit Theorem

TTS - Traffic Technology Solutions, LLC, and Traffic Technology Service, Inc.

TS2VS - Traffic Signal to Vehicle System

IVU - In-Vehicle Unit

UTM - Universal Transverse Mercator

ix 


\section{Chapter 1 Introduction}

\subsection{Motivation}

The transportation sector is partially responsible for the increase in greenhouse gas (GHG) emissions in Canada, which have been climbing since 2009. GHGs are frequently expressed in the common unit of $\mathrm{CO}_{2}$ equivalent. These equivalents are calculated using emissions factors applied to chemical compounds commonly produced by human activities, primarily the combustion of fossil fuels. These compounds trap heat within the Earth's atmosphere, contributing to the warming of the Earth's surface. This warming has caused melting of the ice in the Artic, rising sea levels, severe storms and heatwaves which all have great impacts on the global economy, the environment and human health [1].

Air pollution from traffic has been linked to many negative health effects including lung cancer, heart disease and asthma development in children. The Canadian population is exposed to air pollution from traffic on a daily basis not only while riding in a vehicle or walking along a busy street, but also at home. Strict regulations are in place to decrease greenhouse gas from vehicles, which has largely been possible due to technological improvements. Unfortunately, these measures are not enough to counteract the facts that vehicle numbers are elevated in densely populated areas, and the amount of vehicle kilometers traveled by Canadians continues to increase [2].

In 2015 the transportation sector was the second largest emitter of GHGs in Canada, second only to the oil and gas sector, producing $24 \%$ of the total GHGs emitted in the country. The transportation sector emissions have increased $42 \%$ (corresponding to 51 
megatons of $\mathrm{CO}_{2}$ equivalent [1] ) since the year of the initial working group produced the report of the Intergovernmental Panel of Climate Change which provided scientific evidence of climate change and proposed strategies in response [3]. In December of 2015, Canada signed the Paris Agreement committing to reducing GHG emissions by $30 \%$ below 2005 levels as of 2030 [4]. To meet these goals, the transportation sector will need to significantly contribute in the reduction of emissions.

As seen in Figure 1, of the GHG emissions released by the transportation sector, at least half can be attributed to passenger vehicles year after year. Recently, the amount of GHG emissions produced by passenger cars has decreased. Unfortunately, the increased popularity of "light trucks" such as vans, SUVs, and pick-up trucks has allowed for an increased contribution from this classification of vehicles maintaining a steady trend of emissions produced by personal transportation. These larger vehicles are typically less fuel efficient than cars and as their popularity grows the emissions from this sector will likely increase. Though the fuel efficiency of both classes has been improving continuously over the last few decades, it has not been sufficient to offset this shift in fleet composition [1]. 


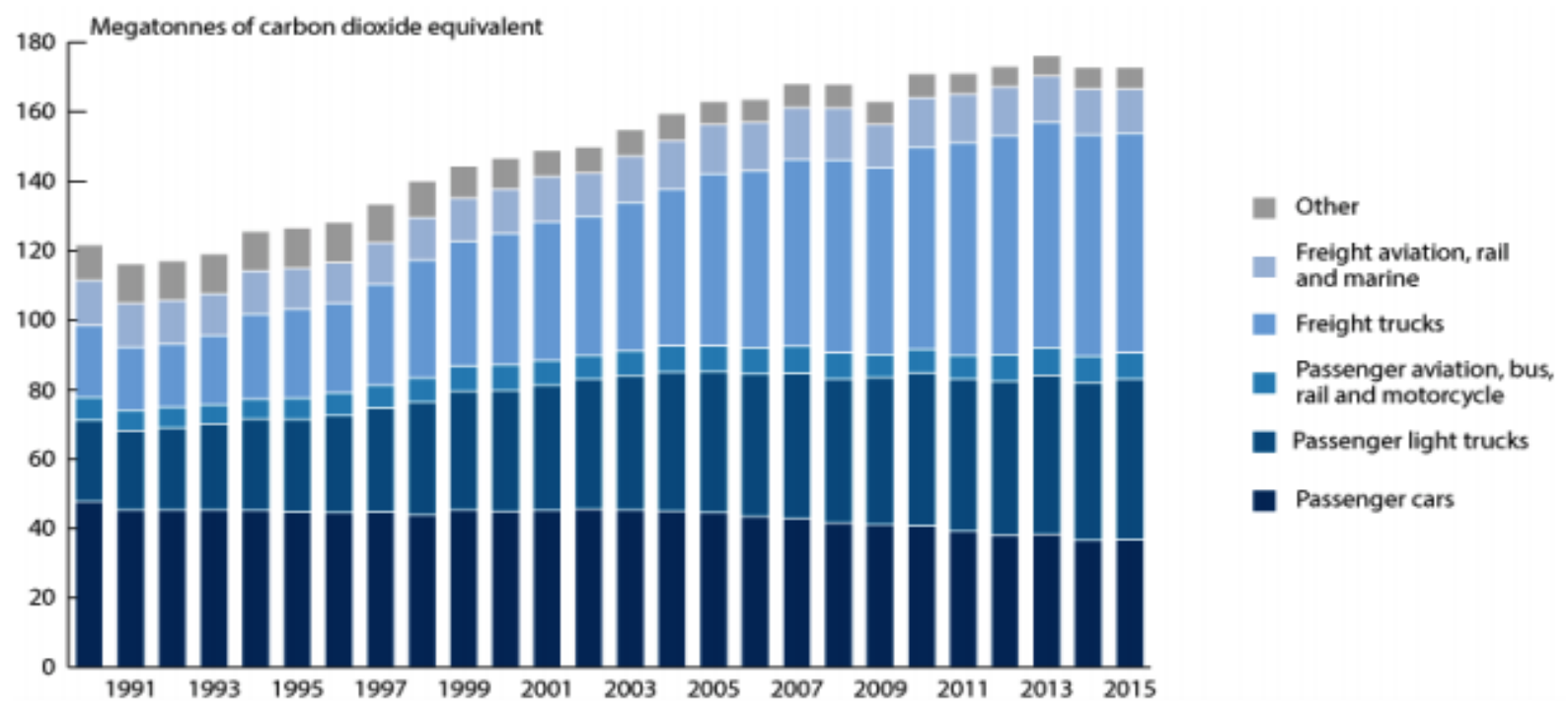

Figure 1 GHG emissions from the transportation sector in Canada from 1990 to 2015 [1]

Globally policy makers have been exploring various strategies for reducing the amount of GHG emissions and air pollution produced by the transportation sector. In addition to regulations and external control measures, much can be done to optimize existing traffic system operations. By leveraging new technologies, the flow of traffic can be improved without significant costs attributed to infrastructure improvements and expansions.

While traffic signals are intended to increase safety and the flow of traffic in all directions, they also contribute to increases in fuel consumption by vehicles idling while waiting to use a crossing. Vehicles then expend more energy and consume additional fuel through the acceleration required to regain cruising speed after being delayed at an intersection. Additionally, momentum is wasted when vehicles are required to brake and come to a stop at a red light. Reducing these braking and acceleration events in traffic networks offers a potential solution to reducing a portion of the emissions produced by the transportation sector through adjustments to the user rather than the system. 
The need for a reduction of the GHGs produced by personal transportation is evident but changing individual's behaviors is not an easy task. The fuel savings attributed to driving behaviours are not enough to compel drivers to change. The evaluation of travel time and the value of travel time savings have been studied extensively [5] [6]. Roadway users place a high value on the time it takes to travel from point a to $b$. This implies that there is a requirement for the development of ways to reduce emissions and fuel consumption which at very minimum do not compromise travel time.

\subsection{Research Objectives}

This dissertation aims to assess the impacts on fuel efficiency, greenhouse gas emissions, and travel time of the use of a connected vehicle technology presenting drivers with EcoDriving recommendations in an urban environment. A comparison is conducted between informed and uninformed drivers traveling along a $6 \mathrm{~km}$ stretch of an arterial roadway comprised of 12 connected semi-actuated traffic signals. A microsimulation, using single second resolution vehicle positional information, is used to produce emissions and fuel consumption estimates for each individual trip. Statistical tests are applied to evaluate the significance of the changes and identify factors impacting these changes.

\subsection{Scope}

The roadway selected is a major arterial connecting the city of Ottawa from east-to-west. A section consisting of 12 semi-actuated, signalized intersections was used for pilot testing of EcoDrive, a connected vehicle technology. The City of Ottawa's 4G LTE network was leveraged to connect the in-vehicle unit (IVU) to a third-party application 
privy to the real-time signal phasing and timing (SPaT) of intersections along the corridor. Testing consisted of passing through over 600 connected intersections.

\subsection{Thesis Contributions}

Rather than conducting assessments through testbed analysis, driving simulators or traffic simulations, this research was conducted over many months in live, real-world traffic scenarios alongside unequipped vehicles on an existing arterial roadway. Additionally, semi-actuated signals were present as a part of the studied route, placing high importance on the requirement for real-time signal phasing and timing information accessibility and quality predictions. State of the art emissions modeling software was used along with single second resolution vehicle positioning information to produce emissions and fuel consumption profiles for all test trips conducted for comparison between the base case and the system use. Statistical analysis was performed to determine the significance of the analysis.

\subsection{Thesis Organization}

The remainder of this dissertation will proceed as follows: Chapter 2 presents background information on traffic network optimization, connected vehicle technology and Ecodriving. In Chapter 3, a review of related research through simulation, field testing on testbeds, and another real-world pilot project is provided. The Hunt Club Road test corridor is presented in Chapter 4 followed by a description of the connected vehicle application, which is presented in Chapter 5. The field testing and preparation of the second-by-second vehicle positioning data through initial spatial analysis are described in Chapter 6. MOVES emission modeling methodology is explained in Chapter 7. In 
Chapter 8 , statistical analysis of the results is presented and these results are evaluated. Chapter 9 presents a summary of the work conducted along with the main findings and recommendations for future studies. 


\section{Chapter 2 Background Information}

\subsection{Traffic Network Optimization at Signalized Intersections}

Though crucial for safety, signalized intersections are a leading cause of delays in urban traffic. They are used to control the flow of traffic at the intersections of conflicting rights of way by giving priority to competing flows which in turn affects mobility. These delays are in the form of vehicles idling while waiting for their turn to traverse the intersection. While vehicles are stopped at traffic signals, they are still running and thus produce tonnes of additional emissions annually. In addition to the emissions produced while awaiting the light change, massive amounts of fuel are consumed and wasted in the approach and departure from these intersections. While approaching a red light, large variations in speed profiles occur as vehicles traveling at a cruising speed comply with amber or red signals. In the approach to a red light, vehicles slow to a stop and upon the signal phase change accelerate back up to a constant cruising speed. It is well known that vehicles expend more energy, and consume more fuel, while accelerating [7]. Avoiding this behavior would greatly reduce the amount of emissions produced by vehicles while traveling along arterial roadways. As a method for mitigating these delays municipalities often employ traffic management teams to developed techniques such as signal optimization to improve traffic flow.

Rather than adding lanes or transit routes to improve mobility, current networks can be optimized to operate more efficiently. Stop-and-go roadways carry only half to two thirds of the capacity of a free-flowing road [8]. It is assumed that $10 \%$ of delays on major roadways in the United States can be attributed to poor signal phasing and timing [9]. 
Cities worldwide have turned to traffic signal optimization and coordination techniques to improve the flow of traffic and thus minimizing some of the above-mentioned inefficiencies of vehicles in their networks.

A basic method of traffic optimization comes in the form of green light synchronization. "Green waves" are created within networks by offsetting the green phase at successive intersections. These offsets allow for the traffic signals along a roadway to be green when the vehicles that had passed through the previous signal would be arriving at the intersection. This permits vehicles on the green wave traveling near the speed limit to pass multiple consecutive intersections on the green phase without having to alter their speed or more importantly stop.

Ideally, traffic signals are timed to minimize stops and delays for all traffic passing through the intersection. Traffic counts have traditionally been conducted through manual means to inform phasing decisions. The manpower required to perform such tasks limits the amount of counts that could take place in a network. Recently traffic counting technology has evolved in the form of computer algorithms which can be run on video or pneumatic tubes. These methods are quite mobile and have made obtaining rich data sets more feasible. Methods are no longer limited to man power, and continuous counts can be conducted over longer periods of time or more frequently. Often, counts are conducted, and factors are applied to infer volumes for certain times of day. Traffic counts and their applied factored volumes are used to inform signal timing plans.

Another optimization technique widely implemented is the use of 'time of day' plans for networks. These plans allow for the system to adjust for fluctuations in traffic 
characteristics. The load on the system is typically different at various approaches during various periods in the day. Common timing blocks are AM peak, PM peaks, weekend and off-peak periods. Various other periods of time can be identified as needed. These periods are typically identified as having unique traffic conditions and are consequently given their own set of signal phase and timing plans. An example of this would be a weekend peak period for signals near retail locations.

Within these plans, actuated signals can be present. Actuated signals only prompt certain phases when they have been triggered. These triggers are most commonly pedestrian cross walk buttons and the detection of the presence of a stopped vehicle. Typically, vehicles are detected through the use of inductive loops imbedded in the pavement. When a vehicle is present over a loop, the metal in the vehicle interferes with the magnetic field produced by the loops and triggers a response. These loops are often present for left hand turning lanes and on minor approaches to avoid these phases occurring while no vehicles are present. At times, it can be assumed that a left hand turning vehicle or two will be able to cross on the amber phase. In these cases, the loop is set back from the stop line to only trigger the turning phase when multiple vehicles are present. Intersections are said to be semi-actuated when inductive loops are only in place at certain, most often least used, approaches. The use of semi-actuated signals increases the capacity of intersections by avoiding unnecessary delays to the major approaches.

With advances in technology, traffic signals can be connected to a centralized location, and traffic optimization can be conducted in real-time. Connecting traffic signals to a centralized controller allows for more control over the network, especially in the presence 
of unusual traffic events such as collisions. Traffic management centers equipped with video cameras can be used to monitor traffic and adjust signal timing as required.

As more advanced technologies become more accessible, though still expensive, traffic can be monitored in real-time through the use of inductive loops or wireless technology. These methods open the doors to more sophisticated optimization techniques one of which is adaptive signal controls. Split Cycle and Offset Optimization Technique (SCOOT), Cycle, Offset, Split MOdel for Seoul (COSMOS) and similar traffic control systems utilize physical sensors built into the roadways to monitor vehicle speeds and real-time traffic states, degrees of saturation or levels of congestion. However, these sensors record and periodically transfer this data to a centralized controller where algorithms are run to adjust the signal phase timing to optimize the network in real-time to accommodate its most recently observed state. These sensors are inconvenient to maintain and come at a significant cost. The trade off to this cost is that these traffic management measures have been proven to function quite well in terms of adapting signals to traffic conditions, reducing traffic delays by about $20 \%$ in urban areas [10].

A large focus on the optimization of current traffic networks has been placed on the adjustment of SPaT settings. Intersections are usually optimized to reduce delays for all users. Although these techniques have been proven to be effective, more can be done to allow drivers to further optimize their trajectories with the provision of additional information. Behavioral changes based on information received regarding signal status allows drivers themselves to adapt and reduce their fuel consumption by limiting speed variations. 


\subsection{Eco-Driving Concept}

Of the variety of strategies that are currently being investigated and implemented to reduce GHG emissions from the transportation sector, one that is gaining momentum world-wide is the concept of Eco-driving. Eco-driving is a blanket term used to define changing driver behavior to reduce fuel consumption and emissions produced while traveling from point A to point $\mathrm{B}$. The name is derived from driving both more economically and ecologically, as saving fuel also reduces emissions.

Changing driver behavior can have great benefits while being relatively low cost in comparison to other emissions and energy reducing driving techniques (such as the use of higher quality fuels or alternatively fueled vehicles). There is no need for significant changes to public infrastructure or automotive production when using many Eco-driving techniques. Eco-driving techniques can be taught through class room education and reinforced with on route or after route feedback. Static Eco-driving programs in Europe yielded between 5 and 15\% fuel economy improvement immediately following the introduction of the topic to drivers [11]. Eco-driving techniques can also be suggested to drivers dynamically through in-vehicle applications, leading to greater fuel consumption reductions.

In Europe in 2013 ECOWILL, a standardized short duration Eco-driving education course, was integrated onto all $28 \mathrm{EU}$ member states category B (personal vehicle) driving certification tests as a result of various pilot training activities that took place in 13 different countries [12]. In many provinces in Canada, efficient driving techniques are taught as a mandatory part of the driver training curriculum, and questions on the topic 
are present on new driver licensing tests [13]. The main components to these programs are gentle accelerations, the anticipation of traffic, avoidance of high speeds, maintaining a steady driving speed, and coasting to decelerate [13]. These components are highlighted in Figure 2 below.

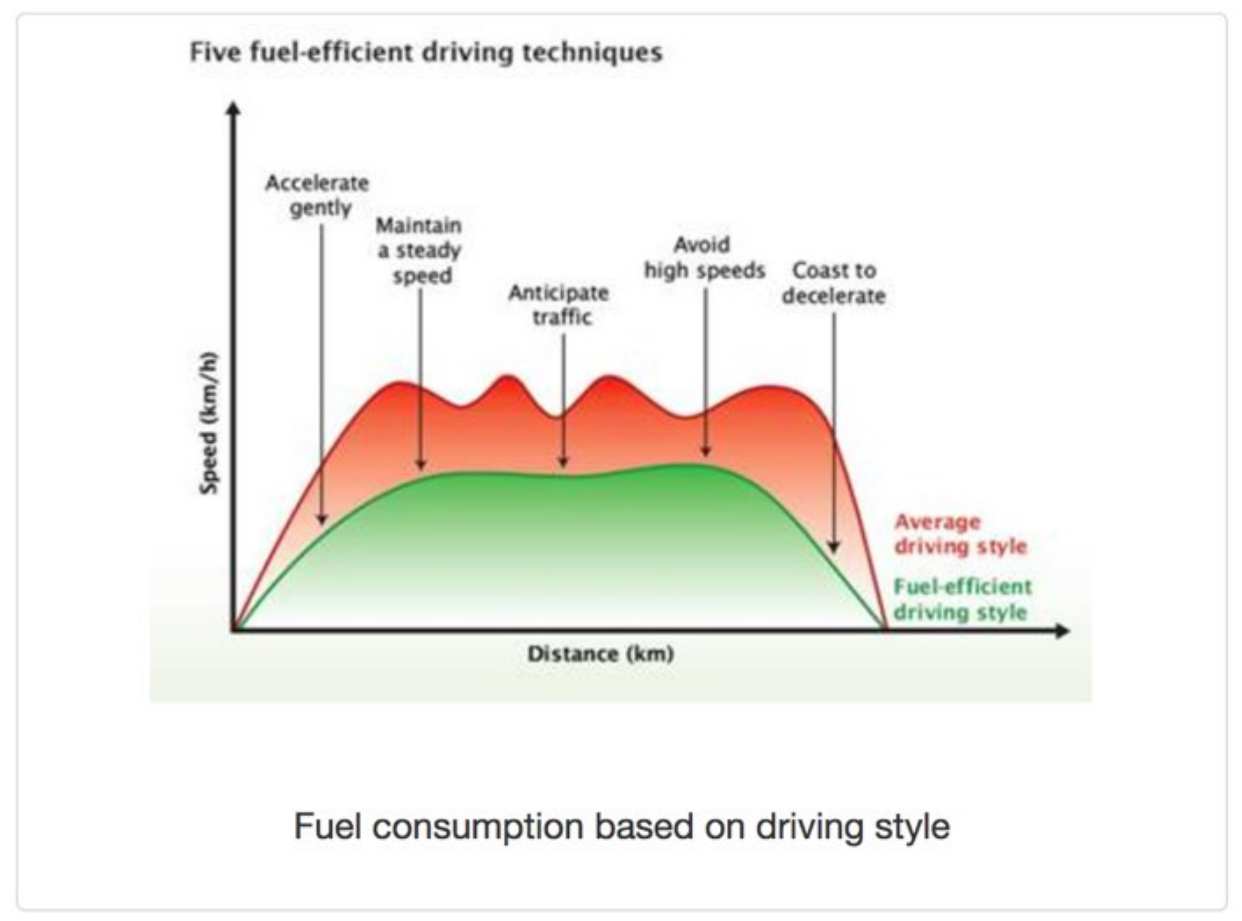

Figure 2: Five components to fuel efficient driving techniques taught in Canada [14]

Hard accelerations, speeding, and frequent braking, better known as aggressive driving styles, can increase fuel consumption by 39\% [13]. Additionally, instead of accelerating hard from a stopped position at a traffic signal, Eco-driving calls for drivers to decrease their speed prior to arriving at the signal by anticipating traffic and potentially avoiding the need to stop entirely. Accelerating back to cruising speed from $8 \mathrm{~km} / \mathrm{h}$ rather than from a full stopped can use up to $20 \%$ less fuel [15]. 


\subsection{Connected Vehicles}

An emerging technology in the automotive industry is the development of connected vehicles. Connected vehicles take advantage of two-way communication through the use of wireless technology to connect with either other vehicles, on-board devices or transportation infrastructure (V2X) [16]. Connected vehicles already frequently travel along our roads with systems such as OnStar ${ }^{\circledR}$ and other wireless mobile internet capabilities. Connected vehicles applications, especially when coupled with sensors, can serve many purposes such as the identification of the location of potholes to be filled and providing red light violation warnings to other drivers approaching intersections. The applications can also facilitate platooning and the use of real-time traffic information in a connected environment could to allow the immediate adaptation of signal controls for optimization [17].

Connected vehicle technology is also being pushed by the development of autonomous vehicles. This connectivity is either useful or vital to the operation of many non-essential automated vehicle functions [18]. This type of communication can alert drivers or vehicles to activities taking place outside of their lines of sight such as signal phasing around a curve or provide assistance with dynamic routing choices. Wider system connectivity would allow vehicles or drivers to obtain more robust information from their surroundings, which would otherwise be unavailable using only their vehicle's sensors, regardless of computational powers.

Coupled with vehicle sensors and connected infrastructure, connected vehicles have the ability to support many safety, mobility and efficiency applications through direct 
transmission of information packages or through transmissions facilitated by connected infrastructure. Not only does this ability to communicate provide many benefits to the users, but also lends itself to the acquisition of rich data sets. The big data provided by the use of connected vehicles could afford much more insight into the world of traffic engineering than has ever been available before and could lend itself to further optimization of traffic networks.

\subsection{Communication Technologies for Connected Vehicle Applications}

There are presently two different base systems being explored to be used for V2X communications. They differ on the basis of the method used for connectivity. The options are either Dedicated Short Range Connectivity (DSRC) or cellular wireless internet such as 4G LTE technology. In previous connected vehicle studies both DSRC and 4G LTE connectivity have been used.

DSRC is a dedicated band of $75 \mathrm{GHz}$ that has be specifically reserved for connected vehicles [19]. This band is similar to that dedicated to Wi-Fi. A benefit of this connectivity method is that a subset of the bandwidth is reserved for safety related messages which require extremely low latency. The biggest shortcoming of this connectivity is the limited range in which it is operational. Range limitations of as low as 190m were seen in the use of DSRC by Xai [7]. The range of DSRC communication can be inhibited due to obstructions such as buildings or trees that are sure to occur in urban environments. The basic range of DSRC communication, uninterrupted, is $200 \mathrm{~m}$ to $300 \mathrm{~m}$ [20]. By contrast, LTE capabilities are theoretically infinite in urban settings. 
4G LTE or other wireless internet technologies span a much larger communication area consisting of whatever is covered by the cellular service range of a given provider. LTE connectivity has the appeal of moderate to low latencies in conjunction with high throughputs. The use of this mode of connectivity is shared with general cellular connectivity, creating an increased demand which may require the development of more capacity [19]. Additionally, the current development of 5G connectivity has the potential to reduce the latency to a level comparable to what is currently obtained through DSRC. Another appeal of LTE technology is that most vehicle operators, especially in North America, already own a device that is able to connect -- smartphones.

Many automobile manufacturers such as Ford, GM, Audi, and Volkswagen, have begun to test and develop connected vehicle technology [21] [22] [23] [24]. Standardization of methods of communication is also important for broad implementation of these technologies. For the development of many of these technologies, some form of connected infrastructure is also necessary, making partnerships between the public and private sectors crucial.

\subsection{Connected Vehicle Technology for Traffic Management}

There have been a few different methods used to approach the idea of using connected vehicle technology to improve the traffic efficiency and mobility: highway platooning [25] [26] [27], electronic tolling [28], effective lane changing and routing [29]. Much of this research has been centered around highway class roadways where the intricacy of traffic signals involvement in the flow of traffic need not be observed. The presence of 
traffic signals forces the requirement to accommodate frequent yet necessary disruptions to the flow of traffic.

Once vehicles are operating in an urban environment, another layer of complexity is added through the presence of traffic control measures and accessibility to all ground transportation modes. In recent studies, many connected vehicle technologies have been developed and evaluated that aim to improve the functionality of intersections. These studies look at ways to improve mobility and reduce emissions. The interchange of information providing the vehicles positions and SPaT and MAP messages plays a major role in making most of these technologies possible. MAP messages provide a description of the physical geometry of intersections. These messages deliver the number of lanes on the approaches to the intersections, the allowable turning movements for each lane, amongst other more detailed information about the intersection geometry. This is a static layer of information that is usually included in connected vehicle technologies to allow for vehicle orientation [30].

The data-rich environment where the location of vehicles traveling along roadways is known in real-time opens many doors for improvement of the traffic networks. One example is adaptive signal timing. This method uses real-time traffic information to adjust signal timing. One way to obtain this information is through the use of sensors on the roadways which can be costly to implement and maintain. Another available method is through connected vehicle and DSRC technology. A case study presented by Feng et al. [31] demonstrated through simulation as much as a $16.33 \%$ reduction in total vehicle delays. For such systems to function properly a sufficient amount of the fleet of vehicles 
using the road way must be equipped with some form of technology, communicating at a minimum the vehicle's current location. Dynamic routing can also be conducted with connected vehicles. Through the use of information from other vehicles on the roadways, the load on a network can be dispersed through dynamic routing. This maximizes use of the network while enabling all vehicles to achieve shorter travel times.

Eco-drive-based applications, on the other hand, have the ability to function with little market penetration. The benefits are seen by each individual vehicle using the technology without the requirement of information of other vehicles on the roadway. These types of systems are more feasible in the short term as they do not require a minimum market share to be functional. That is not to say that the more connected vehicles using such an application would not result in greater benefits, just that it is not necessary for functionality. With an increased market penetration of such systems, these technologies used by some can have a positive impact on the network as a whole. 


\section{Chapter 3 Literature Review}

This section explores relevant research previously conducted that has similar baseline intentions as this EcoDrive pilot and are centered around vehicle-to-infrastructure connectivity. Previous studies have mostly been focused on the development of applications to be used in connected vehicle environments using signal phase and timing. A study is presented in which tests are conducted in a driving simulator. The majority of these studies have used microscopic traffic simulations to demonstrate the usefulness of their application in terms of fuel savings and emissions reduction or travel time benefits. A handful of studies have paired these simulations with field analysis through testing using real vehicles on isolated tests beds. Finally, a single other study conducted in a live traffic environment is also presented. The collection of these studies provides a strong foundation for understanding the work that is currently being done in the field related to the EcoDrive pilot project.

\subsection{Driving Simulator Studies}

Driving simulators are used to mimic the driving environment. Drivers are placed in front of large screens with footage of a roadway, which aims to replicate what would be happening should they be on a real road. Steering wheels and pedals are used to navigate this virtual world.

\subsubsection{Traffic Light Assistance System (TLAS)}

Liu \& Kircher evaluated two different methods for delivering drivers Eco-driving advice through a Traffic Light Assistance System (TLAS) [32]. The primary goal of the TLAS is to reduce fuel consumption with a secondary goal of providing increased driver 18 
comfort, all while not compromising safety. The targeted reduction in fuel consumption is attempted by reducing acceleration events, increasing minimum speed, reducing delays, and having higher speeds while crossing intersections.

The evaluation was conducted using a driving simulator to allow test drivers to pass a series of six signalized intersections, each over a kilometer apart, in a simulated two-lane suburban arterial road with a $70 \mathrm{~km} / \mathrm{h}$ speed limit. Eighteen participants took part completing three runs each: one unassisted trip, and one in each TLAS scenario. The TLAS offered driving advice in two different forms on the screen at about the same glance distance as it would be to a dash mounted device. In the first scenario, the TLAS provided advice in the form of a recommended speed. The second scenario provided drivers with the remaining time in the current signal phase on the screen. When approaching the simulated traffic light, drivers were required to perform one of the following three actions: maintain a constant speed; reduce the current speed to half speed; or, stop at the intersection. The simulation test runs with the use of on board TLAS showed an increased minimum speed, shortened stop durations and higher speeds when crossing the intersections.

The Transportation Research Board in the United States has begun a trial comparing the operation of simulators to real-word applications of Eco-drive technology. A field test took place over an isolated intersection indicating that this type of guidance could reduce fuel consumption approximately $20-40 \%$. This study concluded that a driving simulator was an effective tool for assessing the benefits of Eco-drive systems. It was noted that there was a discrepancy between the fuel consumption values when comparing the 
simulator to the real-world results. Nonetheless, the Eco-drive system outperformed the base-case scenario significantly in both cases [33].

\subsection{Microscopic Traffic Simulations}

In contrast to macroscopic models, microscopic models track the movements and interactions of all individual vehicles throughout the simulation. Microscopic traffic simulations can be conducted on the scale of a roadway or a select set of intersections. These simulations allow for the second-by-second movements of individual vehicles to be simulated and recorded for analysis which become particularly important when modelling emissions. These vehicles move based on a set of predetermined parameters such as driver reaction time, targeted headway times, speed and acceleration profiles, traffic control settings and demand information. Many parameters have been defined within a specific range or can be input with a probability distribution centered on a mean value. These variables are used to construct the case of the average driver and are adapted to simulate the use of the connected vehicle algorithms.

The case of a typical driver used in simulations has been well studied. As outlined in many of these studies, the average driver customarily maintains a speed near the speed limit until they can visually identify that it is not possible to pass the intersection during the current green phase. The driver will then brake as required to come to a stop at either the end of a queue or the stop line. The vehicle will then idle in this spot until the signal changes to green and the driver is able to accelerate back up to the cruising speed, which is typically the speed limit when unaffected by traffic. Base-case simulations are run with 
simulated typical drivers along the same routes which drivers are simulated as using the in-vehicle applications as a means of comparison.

Many studies have used microscopic traffic simulations along with emissions modeling software to demonstrate the effects of changing driver behaviours based on various algorithms in a connected vehicle environment. Simulations model very specific scenarios and often exclude factors such as compliance rates and driver differences in understanding the device and its advice. Despite these shortcomings, these simulations are an excellent step in providing proof of concept of new technologies. Microscopic simulations allow for the effects of multiple vehicles using these applications to be tested, leading to results that provide network-wide indicators at a particular penetration rate.

\subsubsection{Green Wave}

De Nunzio et al. took the approach of allowing a connected vehicle to catch onto a green wave energy optimal trajectory [10]. This simulation assumes that the signal phasing is coordinated and synchronized in a way which will allow drivers to pass multiple successive intersections at around the speed limit. The simulation was run with a 30 second cycle and five intersections to traverse, each $300 \mathrm{~m}$ apart. The maximum speed was set to $50 \mathrm{~km} / \mathrm{h}$ while the ideal speed was set at $35 \mathrm{~km} / \mathrm{h}$. In the above described scenario, at a penetration rate of $40 \%$ connected vehicles, the simulated network offered a $10 \%$ average reduction in fuel consumptions. At a $100 \%$ penetration rate, all stops and idling along the corridor are eliminated and traffic energy consumed is reduced by an average of $28.5 \%$. 


\subsubsection{Connected Vehicle Dynamic Reservation Systems}

Some futuristic thinkers have developed algorithms for potential solutions to traffic management and reducing $\mathrm{CO}_{2}$ emissions including the introduction of entirely autonomous vehicles into the connected vehicle environment. One of these studies is Dresner and Stone's Multiagent Approach [34]. Dresner and Stone present a reservationbased intersection control system where each vehicle entering the intersection is assigned a specific space-time block to enter and cross. Vehicles are not allowed to enter the intersection without a reservation, which means all vehicles within the network must have connectivity. This system worked best at a $100 \%$ penetration rate of connected and autonomous vehicles, with accidents highly present in all other tests. In comparison to a traditional intersection, this system reduces delays by two orders of magnitude, therefore significantly decreasing the fuel consumption and emissions presented by the network.

Jin's Dynamic Reservation System [35] also assumes that all vehicles in the network are equipped and will not enter the intersection without a reservation. In this reservation system, it is highlighted that the algorithm can be calibrated to cater to specific priorities, such as first come first serve or transit priority. The model is built on the concept that reducing the number of stops prior to entering an intersection is a critical factor in reducing emissions and improving travel time. A platoon-based control layer was developed to allow for vehicles which are traveling along the same roadway for multiple intersections to group tightly together, increasing roadway capacity.

As long as human drivers are still partially in control of vehicles or there is a presence of mixed connectivity and automation levels such systems are not the most feasible to be 
implemented. Jin performed further field experiments elaborating on the studies performed by Xai at the Turner-Fairbank Highway Research Centre [7]. These studies made more realistic leaps towards automation and involved a vehicle which was equipped with automated longitudinal control systems.

\subsubsection{Velocity Planning}

A variety of velocity planning algorithms have been developed under many names: Advisory Speed Limit, Green Light Optimal Speed Advisory, Eco-Approach and Departure, Eco-Speed Control. All of these systems use a recommended speed, or a range of speeds, to advise drivers to change their behaviour. The various studies have different approaches and place importance on different factors within the traffic network such as queues and number of lanes. However, all of these systems involve the use of the vehicle's current location and SPaT information of upcoming intersections to produce their recommendations.

\subsubsection{1. $\quad$ Advisory Speed Limit (ASL)}

Ubiergo and Jin present an Advisory Speed Limit (ASL), where drivers are given a speed limit at which to drive to smooth stop-and-go traffic [36]. This advised speed aims to have drivers reduce their speeds ahead of an intersection with a queue, allowing them to arrive at a time when the traffic lights and capacity of the intersection allows for them to traverse the intersection. Leading vehicles, in this case, are included in the algorithm calculation through the presence of simulated inductive loops detectors and a secondary algorithm predicting where those vehicles will end up. This algorithm intends to allow 
vehicles to enter downstream of an intersection at higher speeds to increase approach capacity and maintain smoother speed profiles.

Cellular networks were used as the form of connectivity, and errorless GPS was assumed. Two intersections were simulated with a 60 second fixed cycle and a 23 second split. The simulation did not include any vehicles making turning movements or lane changes though different car following models were examined.

Using the Virginia Tech Micro Emissions Model with a 25\% market penetration in sparse to dense traffic a $2.0 \%$ to $15.1 \%$ reduction in $\mathrm{CO}_{2}$ emissions was observed and fuel consumption was also reduced $2.0 \%$ to $15.0 \%$ (depending on the car following model used). Wait times were reduced in all scenarios from a minimum of $1.6 \%$ at low densities and a maximum of $30.6 \%$ in medium density traffic with a $100 \%$ penetration rate.

\subsubsection{2. $\quad$ Eco-Approach and Departure}

A velocity planning algorithm was developed by Xia et al. with the intention of minimizing fuel consumption through reducing the total tractive power and idling time [37]. Comprehensive Modal Emission Model (CMEM), a microscopic emission model developed by the National Cooperative Highway Research Program and the US Environmental Protection Agency (EPA), was used to produce emission estimates for a light-duty vehicle based on simulated second-by-second vehicle trajectories. This vehicle was modeled along a hypothetical 11 signal, one lane corridor with a speed limit of 65 $\mathrm{km} / \mathrm{h}$. All signals were modeled as having 50 second cycles with 20 seconds of effective green time. Intersections were spaced $500 \mathrm{~m}$ to $650 \mathrm{~m}$ apart. In this scenario, the 
connectivity was based on DSRC connectivity. Therefore, the SPaT timing was only available within $300 \mathrm{~m}$ of the intersections.

A $12.48 \%$ improvement in fuel and $13.22 \%$ improvement in $\mathrm{CO}_{2}$ savings for the single vehicle over the baseline was observed. The simulation was expanded to examine network-wide benefits at different penetration rates with varying traffic volumes. At a $20 \%$ penetration rate, in moderate traffic conditions, a 3.39\% fuel savings was observed in the connected vehicle while $216 \%$ fuel savings was observed network-wide in single lane testing.

Sensitivity analysis was performed on this simulation to identify key factors influencing the results. It identified that as the signal length increased from 90 to 120 seconds, while still maintaining the same portion of green time, the benefits of the system were reduced. When the simulation was expanded to include multiple lanes, the network-wide savings decreased as non-equipped vehicles were able to change lanes to avoid the vehicle using Eco-drive logic.

This algorithm was expanded by Xai et al. to include traffic conditions downstream from the intersection [38]. The expanded algorithm utilizes information obtained from other connected vehicles ahead of the test vehicle. This has great influence when determining the length of the queue at a junction and thus can move the stop line forward, resulting in more accurate speed suggestions. This enhanced algorithm was applied to a simulation of a light duty vehicle crossing three fixed-time signals 500m apart with an effective green phase of 30 seconds on a 60 second cycle. This simulation also shifted the method of connectivity from the previously assumed DSRC to 4G LTE connectivity and was 
modeled to have infinite range. At lower traffic demands maximum fuel consumption reductions occurred at $100 \%$ penetration whereas in high traffic demands the optimal penetration rate shifts downwards towards $80 \%$. The maximum fuel savings occurred at full penetration in low demand and produced a $31 \%$ overall fuel savings. In all cases, the systems with queueing information out-performed the original algorithm.

Sensitivity analysis was conducted on the communications range assuming infinite connectivity is possible. The ability to communicate with a traffic signal $500 \mathrm{~m}$ away allows for time to reduce speed gradually to reach a certain speed to successfully pass the next intersection without stopping. As communication range increases so does fuel savings nearly linearly, until around $600 \mathrm{~m}$ as can be seen in Figure 3.

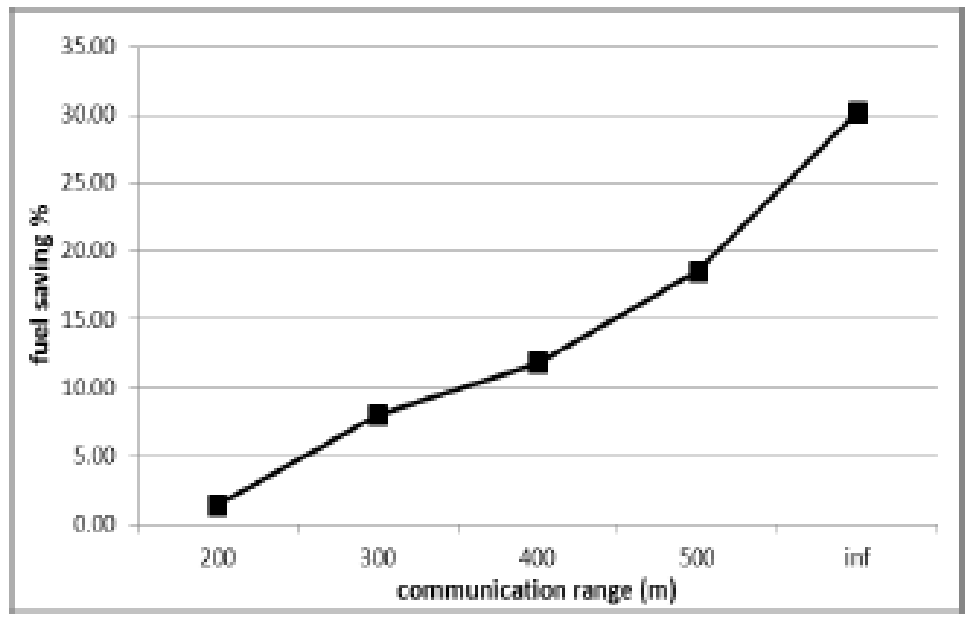

Figure 3: Fuel savings as a function of communication ranges [38]

Finally, Xai developed an Eco-Approach and Departure technique which was intended to be applied to actuated signals [7]. Actuated signals require more than the signal phase timing to be broken down into red and effective green phases as the signal could change last minute with the arrival of a road user. This results in more complex algorithms 
required to be developed to account for this uncertainty. The uncertainty is modeled as probabilities of the length of green phases and the likelihood of arrival within that time frame. Historical data for the signal is suggested to be used to build a profile of possibilities at an intersection. Given the increased complexity of the algorithm, increased importance is placed on not delivering the driver advice that when followed will cause them to experience a red light infraction. Therefore, the algorithm is adjusted to have drivers arrive in guaranteed green time. This approach was not simulated due to initial field testing proving low reliability.

Another velocity planning algorithm with the primary objective of reducing wait times at stop lights and reducing fuel consumption by having drivers always arrive at an intersection in the green phase was developed by Mandava et al. [20]. The algorithm was developed so that in achieving its green phase goal the vehicle is not to exceed the speed limit. The vehicle should, however, cross the intersection as soon as possible while avoiding accelerations as much as possible, and never reach a speed of $0 \mathrm{~km} / \mathrm{h}$.

Simulations were conducted in light traffic conditions on an arterial composed of 10 actuated intersection $500 \mathrm{~m}$ to $600 \mathrm{~m}$ apart. The network's connectivity was simulated to be DSRC assuming a range between $200 \mathrm{~m}$ and $300 \mathrm{~m}$. Along the route, the speed limit was set to $70 \mathrm{~km} / \mathrm{h}$.

Thirty simulations were performed under various link lengths and with random signal phase timing. For each set of conditions, a base case of a typical uninformed driver was run for direct comparison. CMEM was used to estimate the emissions produced by the microsimulation. The results concluded that the fuel consumption and $\mathrm{CO}_{2}$ reductions of 
a midsized sedan were statistically significant while differences in speed were not. When travelling according to the algorithm $12 \%$ less fuel was consumed, and $14 \%$ less $\mathrm{CO}_{2}$ was produced.

\subsubsection{Green Light Optimal Speed Advisory (GLOSA)}

Katsaros et al. developed a Green Light Optimal Speed Advisory (GLOSA) system to optimize fuel and traffic efficiency [39]. This algorithm differs from others presented as based on the distance to the traffic light and current speed the algorithm it would explore the option of accelerating to a higher speed if the vehicle was going to arrive on a yellow. It was assumed when the driver was receiving advice they were $100 \%$ compliant.

The simulation environment created in Simulator of Urban Mobility (SUMO) consisted of a single lane with no overtaking. A scenario was simulated with two intersections, approximately $400 \mathrm{~m}$ apart, along an urban roadway. The speed limit on the roadway was set to $54 \mathrm{~km} / \mathrm{h}$, and a minimum cruising speed of $22 \mathrm{~km} / \mathrm{h}$ was implemented to avoid causing congestion by travelling well below the speed limit. The traffic signal cycles were between 30 and 60 second cycles with 20 seconds of green time. The simulation also assumes that the system is at free flow to begin where there are no queues present at junctions.

At high traffic densities, it was seen that overall stop time was reduced, at times up to $80 \%$, with small penetration rates of connected vehicles in the network. Conversely, to affect the network fuel efficiency, the penetration rate must be at minimum $50 \%$ to be significant. A maximum of a $7 \%$ reduction in fuel consumption was observed over all penetration rates and traffic densities. It was also noted that with less than $350 \mathrm{~m}$ of 
communication range the reaction time required to comply with the driving advice is not sufficient enough to have benefits.

Stevanovic et al. developed and evaluated a GLOSA algorithm on both fixed-time and actuated-coordinated signal phases through simulations in a Vissim C2X platform [40]. The algorithm calculates a speed range in which the vehicle must be traveling to make the light, should the vehicle already be traveling in this speed range it is not given a message. Drivers with GLOSA advice do not react immediately in this simulation and have varied compliance times. This algorithm also will suggest higher speed to the driver to make the light up to 5 miles per hour over the speed limit. Should the vehicle not be able to pass the intersection on the current green phase it will slow, but not stop, to pass the intersection on the following green phase. A test was conducted with minimum advisory speeds, and it was seen that lower minimum speeds provide more flexibility and improve performance.

The route along which the simulation took place was calibrated to replicate an existing arterial with five signalized intersections in Utah. The fixed signal phase and actuatedcoordinated phases were optimized using Vissim-based genetic algorithm optimization of signal timings technology to be compared with the initial signal settings. The GLOSA for the actuated-coordinated signals utilize statistics based on historical signal data at each individual controller. Simulations for each scenario were also run using the case of an uninformed driver for the purpose of comparison.

A light duty vehicle was modeled using CMEM to produce the emissions and fuel consumption estimates. It was found that at higher penetration rates the accuracy of 
GLOSA's queue estimation is increased. Travel times were seen to have improved more in the case with optimized signals than with the use of GLOSA on unoptimized signals. It was also observed that predictions made based off historical mean green time outperform the use of percentiles when dealing with producing GLOSA estimations with coordinated-actuated signals. However, it was concluded that in this simulation with the GLOSA predictions for the actuated signals the fuel consumption and travel time both increase when compared to optimized signals. When the GLOSA was applied to the optimized fixed signal in simulation the travel time was reduced by $1.9 \%$, and the fuel consumption was reduced by $4.4 \%$.

Tielert et al. also produced a velocity planning simulation optimizing approach behaviour [41]. It was assumed that the driver's compliance and the system's connectivity would be perfect. The simulation was conducted along a road with a single 44 second fixed-time signal with 20 seconds of green. The maximum speed limits were set to between 45-55 $\mathrm{km} / \mathrm{h}$ with 50 samples taken randomly from this range. Each speed sample was modeled 44 times, one for each possible variant of traffic signal cycle timing. The maximum speed limits were set to between $45-55 \mathrm{~km} / \mathrm{h}$ with 50 samples taken randomly from this range. A minimum speed of $20 \mathrm{~km} / \mathrm{h}$ was enforced on the driver as to not interrupt the overall flow of traffic. The communication range used in these simulations varied from $200 \mathrm{~m}$ to $600 \mathrm{~m}$. It was seen that greater improvements were observed between increases at shorter distances and that these benefits were maximized between $500 \mathrm{~m}$ and $600 \mathrm{~m}$.

This simulation was modeled in Vissim, and the emissions were estimated with the Passenger car and Heavy-Duty Emissions Model based on a personal car with a diesel 
engine. Average fuel savings of up to $22 \%$ were observed in a single vehicle single simulation. This simulation was expanded to a network of 15 signals where vehicles pass six in a row from the entrance to the exit of the network. A fuel saving in the expanded network case observed a maximum of up to $8 \%$ fuel savings.

\subsubsection{Predictive Cruise Control}

Another technique being developed is the use of predictive adaptive cruise control for velocity planning. This system builds off adaptive cruise control, which is currently in production and a feature present in newer car models. This system will maintain a steady speed or a safe gap between vehicles and is intended to reduce the number of braking incidents and time spent braking, avoiding the excessing incurrence of maintenance costs. Predictive cruise control technology leverages this capability in addition to using the sharing of information through connected vehicle technology to alter the vehicle's speed in a controlled manner based on traffic condition and signal phases. The aim is to reduce idling time while maintaining steady speeds or smooth acceleration profiles.

Asadi and Vahidi [42] developed a model which uses the upcoming traffic signal information to facilitate the automated vehicle system to safely and smoothly either accelerate or slow down the vehicle when approaching a signalized intersection. The system aims to reduce wait times at lights as well as reducing fuel consumption which may potentially have time savings effects. The system's first choice is to increase speed to the maximum allowable to pass on the current green phase reducing idling time and allowing the vehicle to travel further into the network in a shorter period of time. If this is unobtainable, the system will reduce the vehicle's speed to arrive at the intersection in the 
next green phase if feasible through the minimum speed. If avoiding stopping is not feasible the vehicle will approach the intersection slowly to reduce the idling time.

A single vehicle simulation was conducted in Simulink, under light traffic conditions. An arterial roadway composed of 9 fixed-time signal controlled intersections that were spaced a kilometer apart was as the testing facility in this simulation. In comparison to vehicles using traditional adaptive cruise control while traveling for the same amount of time, a reduction of $56 \%$ in $\mathrm{CO}_{2}$ emissions was observed while fuel consumption was reduced by $47 \%$ with the use of predictive cruise control. In this scenario, the connected vehicle was also able to travel further into the network given the same amount of time to travel as the adaptive cruise control equipped vehicle. This study also investigated the use of information for subsequent traffic lights to inform the predictive cruise control decisions. When expanded to a multi-car simulation, predictive cruise control fleets were $4.2 \%$ faster and had a $41.8 \%$ better fuel economy.

\subsubsection{Field Trials}

Some of the previous concepts have been expanded and tested on isolated testbeds. These trials aim to validate the current algorithm. Field trials provide the ability to scale results based on specific real-world scenarios while addressing some issues that simulations do not such as driver reaction time to the speed advice, compliance to the recommended speeds and driver distraction. As was found by Xai [7] the microscopic criteria used in emissions modeling is based on macroscopic traffic parameters and will need to be carefully calibrated using real world data for parameters such as headway times and acceleration deceleration profiles. Only then will the vehicle specific power (VSP) 
distributions from simulations of scenarios modeled with the use of Eco-Approach and Departure type systems be able to capture the microscopic behaviours that would occur in the real world.

Additionally, the drivers in real life do not follow the exact speeds and acceleration profiles as those modeled do in simulations. Field trials are conducted on isolated testbeds, and therefore the algorithms do not need to consider other road users or traffic conditions. The field trials are carefully constructed to replicate certain situations which will highlight the effect of new technologies. Furthermore, some of these field trials have been used to inform other simulations expanding to more complex traffic situations.

\subsubsection{Green Light Optimal Speed Advisory (GLOSA)}

Lebre et al. [43] performed a comparison between GLOSA microscopic simulations and testbed real driving scenarios. The GLOSA algorithm was based on the algorithm presented in Katsaros et al. [39] and used in the European project DRIVE C2X.

The live testing was performed on an isolated testbed $3 \mathrm{~km}$ in length with two equally spaced, unsynchronized, fixed-time traffic signals each with 25 seconds of green time in a 57s cycle. This test in comparison to the uninformed base case exhibited a reduction of $13 \%$ in $\mathrm{CO}_{2}$ emissions.

A simulation environment was built in SUMO to replicate the loop testbed. In the informed driver stage, the object was manipulated "on-line" to mimic the actions of the informed driver in response to the advice given by the application. The simulation showed an $11 \%$ reduction in $\mathrm{CO}_{2}$ emissions produced in comparison to the base case. It 
was deduced that scaling is required to compare simulations to a free flow urban environment.

Further simulations were performed on the same track involving various levels of traffic and penetration of equipped vehicles. The study demonstrated that at a $50 \%$ penetration of traffic signals and equipped vehicles a $30 \%$ reduction in waiting time could be observed. At a $100 \%$ penetration rate, a $10 \%$ network-wide reduction in $\mathrm{CO}_{2}$ emissions was observed. As with the single vehicle comparison, it is assumed that additional scaling of the model would again be required to deduce the benefits in a real-world environment with traffic.

\subsubsection{Eco-Approach and Departure}

Xia developed a dynamic Eco-Approach and Departure techniques surrounding signalized intersections, particularly on arterial roadways, to address the concept creating a way for vehicles to pass signalized intersections using minimal amounts of fuel [7]. This project was the initial stage of the Application for the Environment: Real-time Information Synthesis (AERIS) program initiates by the US Department of Transportation. This algorithm went through multiple iterations and was tested through microscopic traffic simulations as well as field testing on several testbeds. Fuel savings of $10 \%$ to $20 \%$ were seen in both simulation and field testing. An enhanced version of the algorithm in which the downstream traffic conditions were known provided an additional $10 \%$ fuel savings with a maximum of $38 \%$ fuel savings observed.

All field tests of the dynamic Eco-Approach and Departure application were performed under the assumption by the algorithm of a case with a single vehicle uninfluenced by 
other vehicles or external traffic conditions. Each set of tests were conducted in two phases one with the "uninformed" typical drivers and the other with the drivers using the Eco-Approach and Departure on board technology. All speed recommendations were displayed as ranges on a speedometer with green indicating the range of optimal speeds as can be seen in Figure 4.

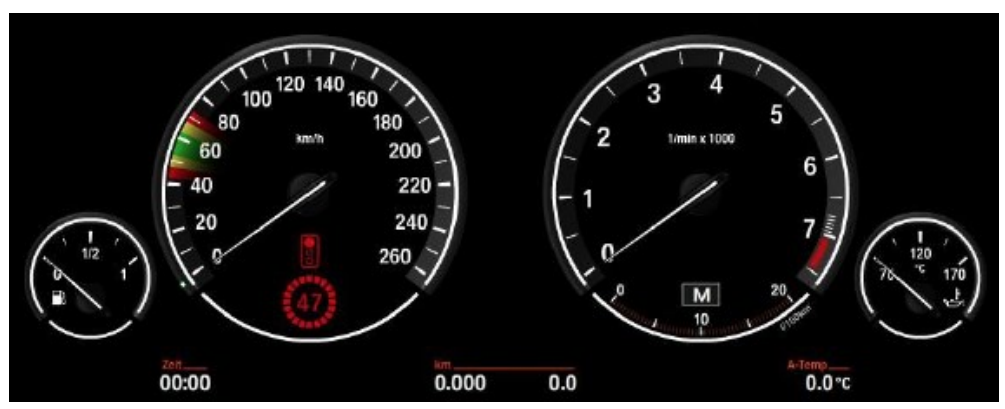

Figure 4: Eco-Approach and Departure human machine interface [7]

The Richmond Field Station tests were conducted first and demonstrated the general concept of Eco-Approach and Departure. The test route was a curved segment of isolated road $307 \mathrm{~m}$ in length with a speed limit of $40 \mathrm{~km} / \mathrm{h}$. The route crosses a single signal with a 60 second cycle, half of which was green time. The communication method used for testing, in this case, was 4G LTE connectivity. The fuel consumption was directly recorded at the source for the BMW test vehicle. When drivers used the Eco-Approach and Departure technology they gained a $13.6 \%$ fuel savings while average driving times remained approximately the same. Generally, the informed drivers had higher speeds immediately after crossing the intersection allowing them to accelerate back to their desired speed more easily to which much of the fuel savings was attributed.

Following the initial proof of concept testing, two field tests were conducted in the summer of 2012 to evaluate the Eco-Approach and Departure algorithm more 35 
extensively. The optimal speed recommendations in this algorithm aim to minimize acceleration and deceleration events. The recommendations were set to be the necessary speed to pass the intersection that is closest to the current speed. In cases where the speed needed to be reduced to avoid stopping the fastest possible speed to be travelled and catch the next green phase was selected. This portion of the algorithm reduces the required acceleration to regain the cruising speed optimizing fuel efficiency. Numerous different approach speeds were chosen coupled with different entry times into the fixed signal cycle.

The second round of field testing took place on the Riverside test bed and at the Turner Fairbank Highway Research Centre (TFHRC). The Riverside field test was along a straight stretch with the use of DSRC connectivity. The vehicle used in this case was a 2008 Jeep Cherokee. The TFHRC testing was conducted on a slightly curved route. The test vehicle in this case was a 2008 Nissan Altima. Due to this curvature, higher speeds of testing $(56 \mathrm{~km} / \mathrm{h}$ and $64 \mathrm{~km} / \mathrm{h})$ were not able to be performed at this site. The TFHRC site was also limited by the terrain including trees that obstructed the DSRC connectivity resulting in a much lower range for the DSRC communication of $190 \mathrm{~m}$ in comparison to the $500 \mathrm{~m}$ available at Riverside.

Both test vehicles had the same equipment and operational capabilities. The signal phases were set to 26 seconds of green time in a 60 second fixed cycle. The field testing was conducted with the conditions of a single lane of traffic, a single vehicle on the track, and the assumption that signal phase timing was known at a $100 \%$ confidence. 
The Riverside testing showed a maximum $28.4 \%$ savings at $35 \mathrm{mph}$ whereas the TFHRC testing experienced the most savings of $18.1 \%$ with the speed limit set to $25 \mathrm{mph}$. This value was consistent with the $17.1 \%$ savings seen at this speed in Riverside. Across all speed ranges at Riverside, a 19.8\% reduction in fuel and $\mathrm{CO}_{2}$ was observed, and at TFHRC an average of $10.7 \%$ savings was observed. These results were consistent with the traffic simulations which showed a $12-16 \%$ reduction in fuel used.

\subsubsection{GlidePath}

A level 2 automated version of the Eco-Approach and Departure prototype has been developed labelled the GlidePath prototype [44]. This prototype is a continuation of the AERIS program's Eco-Approach and Departure at signalized intersection application which saw the development of the previous field testing. The vehicle trajectory planning algorithm utilizes the vehicle's current kinematic state, inclusive of position and current velocity, messages received for a DSRC device, including SPaT and MAP data packages along with system constraints such as maximum acceleration and speed limits to produce the targeted trajectory. The system is capable of handling actuated signal controllers though a green window estimator. This estimator uses advanced statistical techniques of varying levels depending on the degree of actuation at the signals. A scenario identifier is also a part of the algorithm which places the target trajectory in one of four cases. These cases are: maintain speed to make the current green light, accelerate to make the same light, reduce speed to make the following green phase or reduce speed to a stop at the upcoming intersection. 
In this field testing the vehicle used is a Ford 2010 Escape Hybrid. Testing was again conducted at the TFHRC. The evaluation was performed with a fixed 60 second cycle with 30 seconds of green time. The vehicle was tested entering the network at 5 second intervals in this cycle. With the limited range and sight line leading up to their intersection only allows for speeds up to $48 \mathrm{~km} / \mathrm{h}$, testing speeds varied from $32 \mathrm{~km} / \mathrm{h}$ to $40 \mathrm{~km} / \mathrm{h}$. Four drivers, with no prior knowledge of the Eco-Approach and Departure techniques, were recruited to perform the uninformed and IVU informed driver testing phases which consisted of 216 passes of the connected intersection.

CMEM was used to model the emissions produced throughout the testing. Three different testing phases were performed. The initial phase was with the unassisted driver. Secondly, the driver would repeat the testing with the use of the IVU. Finally, in the vehicle with the use of the GlidePath prototype with level 2 automation controlled by the Eco-Approach and Departure system. The IVU informed driver on average obtained a 5\% fuel savings over the uninformed driver which is less than what had been previously observed by Xai. The standard deviation of this result was notably high at $18 \%$. The additional use of the automation resulted in a $17 \%$ average fuel savings over the uninformed driver.

\subsubsection{Eco-Speed Control}

Chen et al. created an algorithm closely related to Eco-Arrival and Departure called EcoSpeed Control [45]. The goal of this algorithm is to create fuel optimized speed profile both upstream and downstream of signalized intersections. The upstream area of the intersection is defined by DSRC communication range and the distance in which the 
vehicle would need to decelerate from the speed limit to a stop without hard braking. In the field testing case, the upstream distance to the intersection was $250 \mathrm{~m}$. Downstream of the intersection the vehicle is set to try to reach the road's speed limit. In the field testing, this distance was a maximum of $180 \mathrm{~m}$ for a total of $430 \mathrm{~m}$ of travel recorded for each trip. The Eco-Speed Control algorithm is directly tied to fuel consumption of the specific vehicle being operated as opposed to controlling factors known to have high effects on fuel consumption. The optimization problem is solved by dynamic programming which takes into account all the combinations of acceleration and throttle values and their corresponding fuel consumption levels.

In this study, audible messages of recommended speeds were communicated to drivers as opposed to the more common approach of visual messages being displayed on a heads up display. This method of communication was used to reduce driver distraction. The target speed was announced to the drivers every two seconds.

The Eco-Speed Control algorithm considers three options when approaching an intersection: accelerate, decelerate or cruise. It currently does not consider neighboring vehicles or potential for lane changes and car following is not incorporated into the algorithm. It is stated to treat the problem as being under light traffic conditions though it could be expanded in the future to accommodate other traffic conditions. Instantaneous fuel consummation levels were reported directly through an On Board Device (OBD). The algorithm's fuel consumption model was based of Virginia Tech Comprehensive Power-based Fuel Consumption Model-1 which was used to validate the actual measurements and to calculate the optimum speed profile. 
Controlled field tests took place on an otherwise empty testbed with one lane in each direction. The roadway had a speed limit of $64 \mathrm{~km} / \mathrm{h}$ with a single four way intersection. The test vehicle was a 2014 Cadillac SRX. Four drivers conducted 192 trips under four different red phase signal timings, each between 10 and 25 seconds in length all with a 25 second green phase.

This study split its trips for analysis into downhill and uphill trips, depending on the direction traveled on the testbed. Either direction had a 3\% grade. This field test resulted in an average fuel savings downhill of $26 \%$ and an average travel time reduction of $7.1 \%$. Uphill saw less of a fuel savings at an average reduction of $8.8 \%$ but a slightly higher travel time savings at an average reduction of 9.7\%. It was noted that with the Eco-Speed Control system the vehicle reduced their speed less while approaching a red light and thus were able to accelerate back to the speed limit on the other side of the intersection more quickly in many cases.

Field tests further the proof of concepts that have developed through modeling and introduce numerous factors which are smoothed out in simulations such as latency, communication reliability, how drivers perceive the information and use it to adjust their behaviour. A whole other layer of complexity becomes involved when these vehicles and drivers are required to interact with other drivers out on public streets.

\subsection{Real-World Testing}

Through simulation, it has been studied how connected velocity targeting technologies may behave in real-world scenarios. The majority of these scenarios study the effects of specific penetration rates controlling the entire fleet using the network either on single 
lanes with no passing or with car following models. The real world is full of unpredictable factors and events which provide the need to pilot these technologies as they interact in real-time with other unequipped road users. Live testing accounts for the driver experiencing pressures of driving below the speed limit from surrounding vehicles and driver distraction by posted speed limits. Various drivers also have different thresholds for what is an acceptable level of acceleration and deceleration. Additionally, with prolonged use of new technologies drivers are likely to become more comfortable and potentially less compliant. It was seen in many cases with static Eco-drive training that after a few months the driver's application of the techniques learned had significantly dwindled despite the knowledge of the benefits [46] [47].

As it is an emerging field of study, very few publications exist where the connected vehicles have been taken off testbeds and into real-word scenarios.

\subsubsection{GLOSA In Vehicle Traffic Signal Advisory System (ITSAS)}

Lee et al. conducted real-world testing of an In-Vehicle Traffic Signal Advisory System (ITSAS) [48]. The intent of this system through the provision of real-time speed advisories is to minimize delays when crossing intersections. ITSAS uses cellular network services to communicate with a smartphone or a tablet IVU. Testing was conducted along a $10.5 \mathrm{~km}$ stretch of urban eight lane highway, A-1, in New Jersey. Along the corridor, no left turns are permitted, and the at grade intersections are controlled with coordinated-actuated signals. These signals are handled in the ITSAS by monitoring historical green time changes, for the same period of day and week, and providing probabilities to estimate green times for each cycle second. The speed limit 
along the corridor is $88 \mathrm{~km} / \mathrm{h}$. This corridor was chosen based on its traffic, surrounding land use characteristics and the availability of real-time traffic signal information. The route consists of coordinated-actuated signalized intersections.

The ITSAS had been developed as DSRC free connected vehicle application to allow for more cost-effective implementation and rapid acceptance. The mobile application, which exploits existing user technologies such as smartphones and tablets, also collects instantaneous speed, position and acceleration rates. Typical smartphone GPS systems are subject to $5 \mathrm{~m}$ to $7 \mathrm{~m}$ error though that was not seen to be a problem as the advisory speed was initiated $200 \mathrm{~m}$ to $400 \mathrm{~m}$ from the intersection. Vehicles being required to stop would be provided with proper distance to do so regardless of the GPS unit error.

The ITSAS manager receives positional information from users as well as current signal timing data from the traffic signal controllers. These traffic controllers are connected to a staging server for the safety of the system. This data is transmitted from both sources to the ITSAS manager at single second intervals to compute real-time speed recommendations.

The boundary for acceleration of the system was set to $+/-3 \mathrm{~m} / \mathrm{s}^{2}$ due to factors such as driver comfort, vehicle performance, and traffic safety. If accelerations within this range were possible to make the next a green light, the ITSAS would recommend the driver to do so. Otherwise, drivers are either informed to prepare to stop or are given a range from a minimum speed to the advisory speed based on acceptable acceleration rates. The application's display provides the driver with both a speedometer on which the speed ranges are displayed and the remaining time in the current signal phase. 
Field testing was broken down into three different timeframes. The peak flow was designated as 8 am to $10 \mathrm{am}$, midnight was designated as the hours of $10 \mathrm{pm}$ to $12 \mathrm{am}$, allowing close to free flow traffic, and the Off-Peak period of $1 \mathrm{pm}$ to $5 \mathrm{pm}$ was chosen as a mid-range congestion period. Three to four data collection cycles took place per hour during each period of testing and each period was tested a minimum of three times. The base case for comparison was constructed through crowdsourcing data from GPS based devices and cellular networks for the corridor on which testing took place.

The travel time savings were reported as 3 minutes on average though there were no statistical results presented to verify the significance of these outcomes. The standard deviations ranged from 2.3 minutes to 1.8 for the time of day testing periods. The assumingly smoother distribution of crowdsourced data points also provided no indications of reliability. Travel time savings were mostly attributed to confidence in changing lanes.

The aforementioned literature has demonstrated that there is an excellent potential for benefits to be seen through infrastructure-to-vehicle connected systems. Many simulations have been conducted, some with the addition isolated field tests to inform simulation adaptation and algorithm improvements. The studies that have been conducted by field trial have shown that there is a required scaling of simulated results to account for the uncertainties in the real-world application of these technologies. Few simulations were implemented on routes with actuated signals though mixed results were presented. The majority of these reports have thoroughly analyzed the potential effects of connected systems and have developed technical solutions for optimized algorithms that will likely 
improve fuel consumption, the efficiency of driving and/or travel times. However, none of these studies have evaluated fuel efficiency and emissions reductions through the realworld use of a traffic signal connected in-vehicle application. 


\section{Chapter 4 Corridor Definition and Study Description}

\subsection{The Hunt Club Road Corridor (HCRC)}

The corridor on which the study took place is a $6 \mathrm{~km}$ stretch of an urban arterial roadway, Hunt Club Road, in Ottawa, Ontario. The road is four lanes wide, and for the majority of the route, the roadway is divided. The section of roadway consists of 12 traffic signals, shown in Figure 5, that were equipped to communicate, through a third-party application, with the Samsung tablet IVU.

Three of the 12 intersections on the corridor are crossing other urban arterial roads:

Riverside Dr., Prince of Wales Rd., and Merivale Rd. Along the east end of the route is predominantly residential developments with two signalized intersections connecting the urban collectors: Uplands Dr. and Paul Anka Dr. to Hunt Club Rd. The remainder of the intersections are industrial or commercial access points.

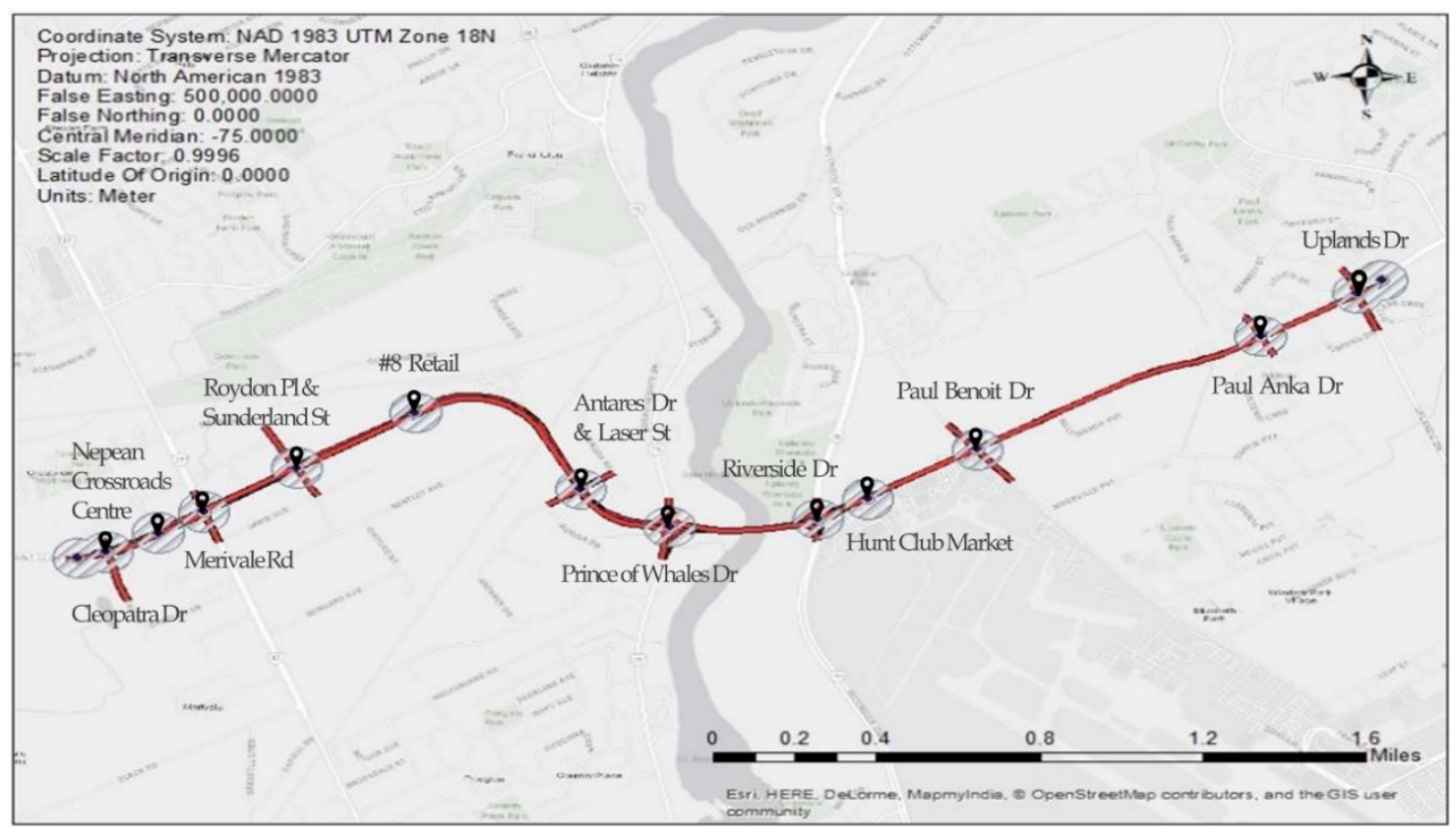

Figure 5: Intersection locations on the Hunt Club Road Corridor 
The majority of the Hunt Club Road Corridor (HCRC) is serviced by three regular public transit bus routes, 92, 96 and 197, with the addition of two peak period bus routes, 199 and 198. From Merivale Road to Antares Drive is serviced by a local bus, route 83 . As the corridor has at least two lanes at any given point in either direction, the presence of public transit busses and stops along the corridor did not interfere with the testing unless traffic volumes were too elevated to allow for lane changes when following a bus. In these cases, the traffic would have been considered unusual in comparison to other testing periods.

In addition to being a major arterial in the city, the roadway is also a designated trucking route and possesses bike lanes. At grade bike lanes are present on both sides of the street along the majority of the corridor with the exception of between the intersections of Riverside Drive and Paul Benoit Driveway. The trucking route is one of five options for trucks to cross the city from east-to-west while avoiding the down town core. The route also has sidewalks on both sides of the street except between Antare Drive \& Laser Street and Roydon Place. \& Sunderland Street. Additional Transportation maps can be seen in Appendix C.

Traffic volumes were measured along the route throughout the study. Three locations were chosen, and the count was taken at least five times on weekdays during September and October of 2017. The traffic counts were conducted for an entire 24-hour period. These traffic counts were produced through the use of Miovision Technologies Inc. traffic technology acting on video of these points recorded by the City of Ottawa. The mean daily traffic for the three different locations is presented in Table 1, which shows a 
variation of over 17,000 vehicles a day between the center of the route and the two collection locations towards either end of the route. The level of service on the roadway varies quite vastly throughout the day, especially in the vicinity of other arterial roadways. At peak periods sections of the roadway can become congested, and traffic is stop-and-go.

Table 1: Mean week day traffic volumes along the corridor during the study

\begin{tabular}{|l|l|}
\hline Location & $\begin{array}{l}\text { Mean week day } \\
\text { motor vehicle traffic } \\
\text { volume }\end{array}$ \\
\hline Hunt Club Rd. Between Paul Anka Dr. and Paul Benoit Dwy. & 35697 \\
\hline Hunt Club Rd. Between Riverside Dr. and Prince of Wales Dr. & 57667 \\
\hline West Hunt Rd. Club Between Roydon Pl. and Antares Dr. & 40891 \\
\hline
\end{tabular}

\subsection{Traffic Signals}

In the City of Ottawa, all the signals are connected to local traffic controller boxes which communicate with the Ottawa Traffic Management Centre. In the centre, operators are able to monitor traffic conditions through real-time video images. The operator is able to immediately adjust traffic signal timing and phasing when an incident or disruptions to regular traffic patterns occur for the majority of intersections (City of Ottawa, 2017).

In normal traffic conditions, the SPaT plans in the city of Ottawa typically have five basic periods for timing plans: the AM and the PM peak periods as well as off-peak, night and weekend periods. Additional plans are developed for specific intersections to accommodate special conditions such as high fluctuations in traffic flows on weekday 
mornings. These extreme fluctuations cause the need for various AM rush period timing plans. The intersection of Hunt Club Road and Riverside Drive, for example, has 15 different time of day plans, though only two are active during the times in which the study took place. The active signal timing plans during the EcoDrive testing time frames consist of cycle lengths from 70 to 150 seconds [49].

In the development of signal phase and timing plans by the City of Ottawa, when an ideal solution is not available the City tends to favour the direction in which heavier traffic is present. These techniques often coordinate signals along busy routes to allow for vehicles to get onto green phases, allowing them to continue at or near the posted speed through multiple successive intersections. The City of Ottawa updates major corridor schedules annually and will intermittently make changes as a response to public inquiries or observed traffic pattern changes [50].

In addition to optimized traffic signals, actuated signals are deployed throughout the city and in particular along the HCRC. These systems use inductive loops embedded into the pavement to trigger a response to a waiting vehicle. In the case of many of the intersections along the HCRC, inductive loops are used at minor approaches and to facilitate the protected/permitted left hand turning phase of a signal. For intersections with protected/permissive left turns along the corridor the loops are set back either 8 or 14 meters to cause the signal to only be adjusted depending on the number of vehicles in the queue. The activation of protected/permitted left-hand turning phase usually improves the safety of the turning vehicles, but generally reduces the capacity of the intersection as a whole. The City implements inductive loops at certain locations to allow for increased 
capacity when there isn't a need for the additional phase, which would otherwise block the through movement of vehicles travelling in the opposing direction [50].

Another form of actuation in the city is done through the pedestrian cross walk buttons. These change the amount of green time relative to the amount of time required to cross the road on foot as opposed to the green time required by vehicular traffic. Along the HCRC there are intersections that span six lanes whose signal phase timing could be significantly affected when pedestrians are crossing.

\subsection{Speed Limit}

The posted speed limit changes once along the east end of the test route near to the residential areas. In the section from Uplands Drive to 600m west of Paul Anka Drive when heading westbound or 700m east of Paul Benoit Driveway when driving eastbound, there is a posted speed limit of $60 \mathrm{~km} / \mathrm{h}$. On the remainder of the HCRC, the road has a posted speed limit of $80 \mathrm{~km} / \mathrm{h}$.

\subsection{Sample Size Requirement}

As a part of the assisted commercial vehicle EcoDrive pilot project, Carleton University deployed a test van (the Van) as a means of calibration. In order to have the results of these test trips be significant the minimum number of required trips needed to be determined.

As per Central Limit Theorem (CTL) the sampling distribution of the sample mean is acceptably approximated to a normal distribution when the number of samples is sufficiently large. CTL holds no matter the shape of the population distribution. 
Typically, this sample size is said to be greater than 30 if the population distribution is not too skewed. Should the distribution be significantly skewed, such as an exponential distribution, the minimum acceptable sample size would have to be greatly increased from 30 . However, if the sample distribution is normally distributed, a smaller number of samples is acceptable [51].

Erring on the side of caution, the assumption was made that the sample distribution would not be perfectly normally distributed and that more than 30 samples would be required. This assumption was verified using Equation 1 to estimate the minimum acceptable number of samples required. The intention was to have enough samples to have statistical significance at $95 \%$ confidence level. Therefore, the standard normal deviate of 1.96 was used. It was assumed that the average speed on the corridor would be $40 \mathrm{~km} / \mathrm{h}$ with a $5 \mathrm{~km} / \mathrm{h}$ standard deviation (s). This assumption was made as an educated estimate after having travelled the corridor multiple times while performing quality assurance on the equipment before the beginning of testing. The tolerance was chosen as $1.75 \mathrm{~km} / \mathrm{h}(\mathrm{e})$. These values produced a minimum acceptable sample size of 31 trips. A sample size of 50 trips for each condition being evaluated was chosen as there was likely going to be some trips that would be required to be removed as outliers.

Sample size computation:

$$
\text { Equation } 1 N \geq \frac{1.96^{2} * s^{2}}{e^{2}}[52]
$$




\section{Chapter 5 EcoDrive System Architecture}

The City of Ottawa retained professional services of Traffic Technology Solutions, LLC, and Traffic Technology Service, Inc. (collectively TTS) to develop a Vehicle-toInfrastructure (V2I) application to function with the 12 enabled signals along the HCRC as a part of the Assisted Commercial Vehicle Eco-Driving Pilot project. TTS was responsible for the capability of the equipment, data storage and the transfer of data to Carleton University for evaluation. The V2I application was developed to produce realtime predictive SPaT information, based on live signal status information provided by the City of Ottawa Traffic Services, to display eco-friendly driving recommendations.

TTS has developed a core system, the Personal Signal Assistant, which functions with the support of their Traffic Signal to Vehicle System (TS2VS) [24]. This system was developed through the use of patented methods and algorithms which predict signal timing changes for actuated signals with the use of real-time SPaT information. The system then generates predictive SPaT and MAP messages in a format that complies with the SAE J2735 industry standards. TTS's TS2VS system was developed to function with North American style traffic control systems which use coordinated-actuated signal controls. The TS2VS system consists of two major communication systems. The backend system is used to predict near-future signal states at actuated-coordinated intersections in real-time. For accurate signal state predictions, the following real-time data is required: timing plan numbers, phase status, cycle second, vehicle and pedestrian call status. The frontend system handles the in-vehicle EcoDrive advisory application. This system aims 
to reduce fuel consumption, emissions, and the number of stops made, while also reducing driver anxiety.

In a previous project, TTS partnered with Audi in Germany to deploy an adapted version of this system at 15 intersections. It was required that they modify their TS2VS to function with German style traffic control signals including the use of transit signal priority. This system was deployed over the course of nearly a year period, May 1st, 2016 to Mar 31st, 2017, where the real-time predictive signal phasing was communicated to a fleet of vehicles though cellular data communication. Another adaptation of this system is currently deployed as Audi Connect in specific models of Audi vehicles sold within the United States. This system's integration into the vehicle system was the first commercially available vehicle-to-infrastructure product deployed in North America [24].

A local deployment of a customized version of the Personal Signal Assistant system was installed on a server at the City of Ottawa's Traffic Management Center. This co-location allows for the system to interface with the City's signal control system behind the City's firewall. The established central connectivity was essential for the dissemination of realtime driving advice. Figure 6 provides an overview of how information is communicated within the system for this project. 


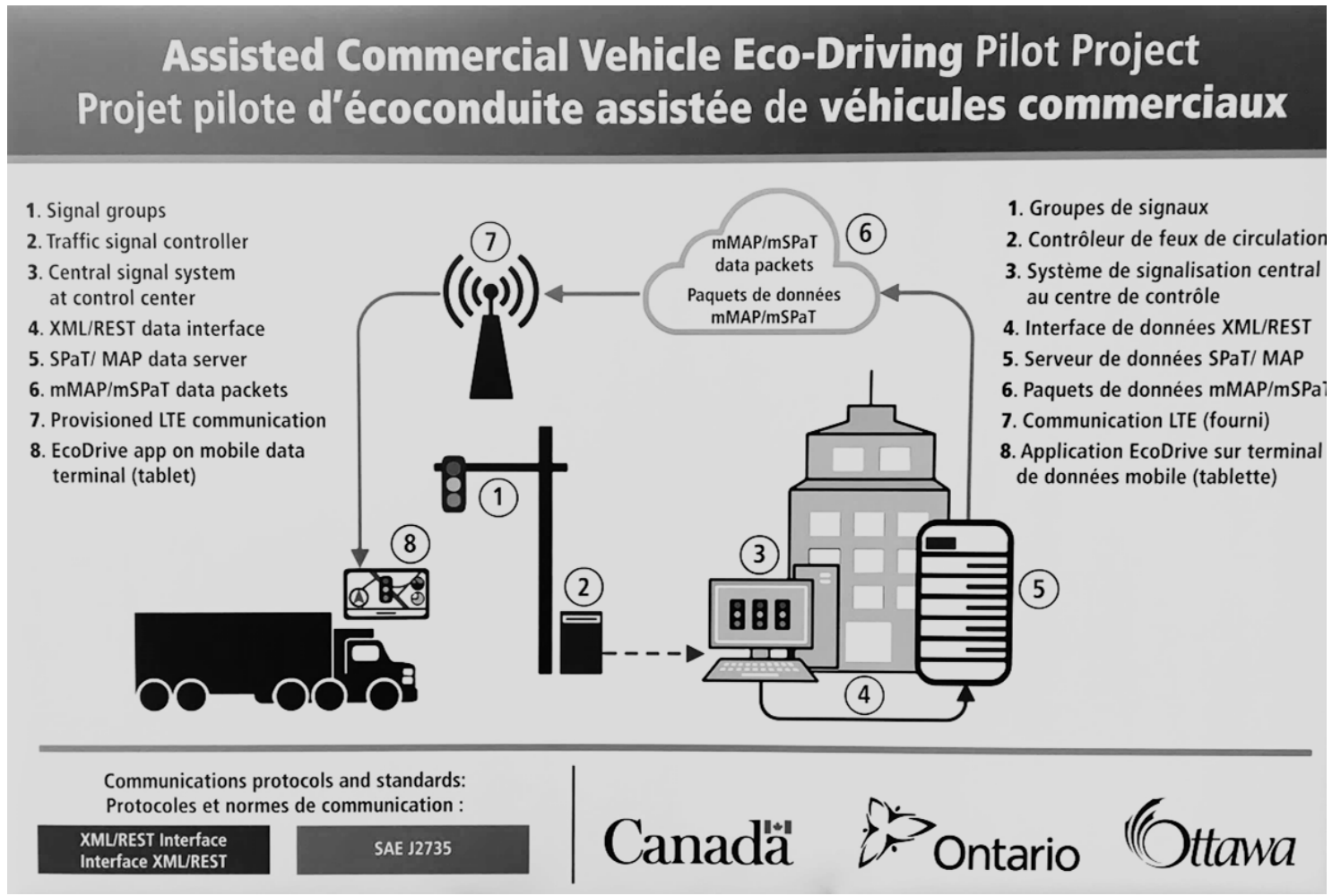

Figure 6: High-level EcoDrive system architecture [53]

The IVU for the project came in the form of an Android based Samsung Tablet enabled with a City provisioned LTE SIM card operating on the City's private Access Point Name. This SIM card provided the capability for the IVU to possess 4G LTE connectivity. The SIM card also provided the necessary security authorization to allow the IVU to receive signal prediction information from TTS' Personal Signal Assistant system behind the City's firewall. The utilisation of readily available technology, commercially available mobile devices and existing wireless networks allowed for this study to be implemented at a much lower cost and more rapidly than many of the proposed V2I systems in the literature which require road side units and DSRC-enabled devices. 


\subsection{EcoDrive Frontend Application}

Depending on the current position of the vehicle, the IVU displayed one of four messages, as seen in Figure 7.The EcoDrive application foresaw four different scenarios which could occur while using the application [54]. The first scenario was that at the upcoming intersection the current phase was green and upon arrival at the stop line the signal would remain green. In this case, the driver was provided with a GLOSA speed recommendation, at or below the road's speed limit. This speed allowed for the vehicle to pass through the next intersection on the current cycle's green phase. These messages were shown in a similar fashion to the posted speed limit signs along the corridor. This message, seen in the furthest left side in Figure 7, consists of a white rectangle with the recommended speed written in black numbers in the centre.

The second scenario is described as the current signal phase being green but would have changed to amber or red (following the amber) before the vehicle arrived at the stop line. In this case, the driver would again see a white rectangle, but in this case, the rectangle would have a black " $X$ " in the centre, seen as the symbol second from the left in Figure 7. This message indicated that a stop was inevitable. At the appearance of this message, the driver would coast when possible towards the intersection. Should the driver have been far enough away that coasting was not an option, he or she would continue at a steady speed until coasting became an option. This behaviour was intended to avoid unnecessary braking and acceleration activities while avoiding causing increased congestion due to travelling well below the posted speed limit [54].

In some scenarios where there are long distances between traffic signals, the upcoming intersection is far enough away that a full signal cycle will pass before the driver arrives. 
The EcoDrive application only perceives the current signal cycle. In these cases, the driver will be told they will not make the light in the current phase. This is a case where the drivers would continue at a steady speed until they are within range of the intersection before coasting to a stop. Occasionally, over long distances, the signal cycle will change placing the vehicle back in scenario one or three and the EcoDrive recommendation will change.

The third scenario potentially present on the roadway was that the current signal state was amber or red but would have changed to green by the time that the vehicle would have arrived at the stop line should they follow the EcoDrive advice. In this case, similar to case one, the driver will be given a GLOSA recommendation at or below the speed limit. This speed would have the driver arriving a safe distance from the stop line, using a maximum deceleration rate of $3.42 \mathrm{~m} / \mathrm{s}^{2}$, a few seconds after the signal had turned green. This additional time with the green signal active, prior to the arrival of the vehicle, is intended to allow any queue which was present to have dissipated. This is one of the cases with the greatest potential for energy savings and emissions reductions as accelerating to cruising speed from a low speed requires less energy than accelerating from a full stop.

The final potential scenario is described as having an amber or red signal which would remain in a red signal state upon the vehicle's arrival at the stop line. In this case, no GLOSA recommendation can be computed to allow the vehicle to pass the intersection without requiring the vehicle to stop. Therefore, the driver would be shown the time to green, represented by the third symbol from the left in Figure 7. To avoid confusion with 
the speed recommendations the time to green information was displayed in a red circle with red numbering. This numbering would count down the number of seconds remaining until the signal phase would change to green. This portion of the application was intended to reduce driver anxiety by providing the ability to see when the next cycle would have begun.

A fourth and final message that would appear on the IVU would be a white question mark surrounded by a white circle. This symbol would mean that the information at the upcoming intersection was not currently available at an acceptable level of confidence or that the communication signal had been dropped. The operator would then continue to drive as they normally would when not receiving external guidance.

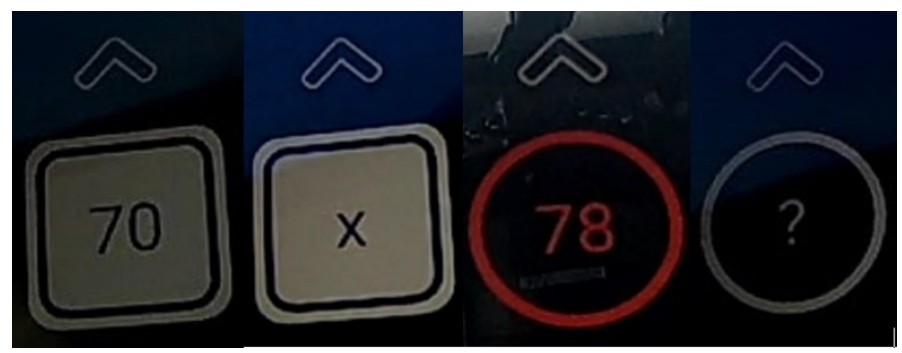

Figure 7: IVU display message options

Using the MAP information, the IVU displayed up to three different messages at a time to allow the driver to be informed for whichever approach, left-hand turn, straight, or right-hand turn they may be intending to use. The direction or directions that apply to the message are indicated by triangular symbols above or beside the message, as can be seen in Figure 8. 


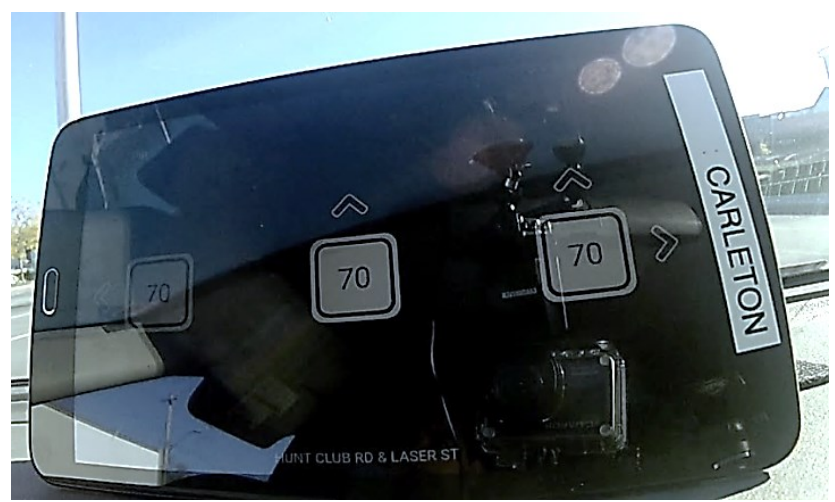

Figure 8: IVU display as seen during testing

\subsection{The Instrumented Carleton Van}

Along with being equipped with the IVU, the Instrumented Carleton Van ("the Van") used for this study was also equipped with a GPS unit, front and rear laser guns, and two front facing video cameras. Photos of the Van can be seen in Appendix D.

The IVU was mounted to the driver's right on the dashboard. This location was chosen to allow the driver to view the displayed messages with minimal head movement while not obstructing the driver's view of the road.

The GPS System was composed of a LEICA SR520 GPS receiver and a 500 AT501 antenna. The antenna was fixed to the roof of the Van $1.4 \mathrm{~m}$ above the ground. The antenna was wired to a LEICA SR520 GPS receiver which was fixed to a structure built where the passenger seat would normally have been. This receiver, which processes the GPS signal received by the antenna, allows the coordinates to be calculated with a precision of about $0.5 \mathrm{~m}$ [55]. This unit was recording the vehicle's position on a secondby-second basis to a PC card. The PC card information was then transferred and consolidated on greater capacity SD card which at the end of each day would be transferred to an external hard drive. The GPS unit allowed the capture, on a microscopic 
level, of the trajectory of the vehicle travelling in real-world traffic and driving conditions. This would later allow for the instantaneous operating speeds, accelerations and roadway grade to be determined at each point, fulfilling the requirements of the emissions modeling software.

The laser guns were used to measure the distance to either leading or following vehicles and time stamp the headway samples. The back laser was operated by an operator seated in the rear seats of the Van. The front laser was fixed to the structure where the passenger seat would have been, along with one of the cameras and the GPS receiver.

The first camera, fixed to the passenger seat area, observed traffic conditions through the windshield as would have been seen by the driver. This camera could later be used to verify any irregularities that appeared in the GPS data such as disruptions in flow caused by the presence of accidents or road work. The second camera was set to observe the IVU for reference. Both cameras' videos were timestamped.

The Van required three operators to be on board at all times. The required operational positions were a driver, a front laser operator, and a rear laser operator. The two laser operators also made qualitative observations about surrounding driver behaviors, driver distraction by the IVU and system accuracy.

\section{Chapter 6 Data Acquisition and Formatting}

\subsection{Data Acquisition}

The Van used to perform testing was commissioned to be used as calibration for the commercial vehicle fleets awarded the contract to conduct the City of Ottawa Connected 
Commercial Vehicle EcoDrive Pilot project. The use of this vehicle as calibration required trips to be performed during business hours to allow for comparison. Only offpeak hours were used to conduct the testing as peak-periods allow for little conformity to speed recommendations. The Van was driven along the corridor on weekdays over a period from September to October 2017.

All tests were performed under dry roadway condition in clear weather. The testing took place over seven days. A total of 120 trips of the HCRC were conducted. All of the trips performed as a part of the testing passed through all 12 equipped intersections successively. U-turns were executed at the subsequent intersections following the end of each trip, creating a 14-intersection loop. This allowed the Van to properly clear the final intersection and for the effects of the EcoDrive application on the initial intersection to be observed.

Testing was conducted in two phases. The initial phase was conducted without the use of the EcoDrive application, and therefore the driver is considered to have been in an "uninformed state." A total of 50 trips were conducted in this phase of testing. The second phase of testing was conducted with the EcoDrive application active on the IVU creating an "informed" driver state. A total of 70 trips were performed with informed drivers. Overall, 600 intersection crossings were performed with drivers in the uninformed state and 840 connected intersections were crossed by an informed driver. The number of informed driver trips is significantly higher than the uninformed driver trips for reasons explained in the following chapter. 
Testing was set to occur between the hours of 9:00 am and 3:30 pm, to avoid operating in either the AM or PM peak periods. The hour between 12:00 pm and 1:00 pm was also set to be avoided due to the unpredictable increases in traffic volumes that occur sporadically along the corridor at this time. This hour was taken as an opportunity to recharge the equipment and perform data downloads. The time span over which testing was conducted crossed though different time of day signal plans along the corridor as the city implements multiple plans for different times of day and previously observed traffic variations.

Three different drivers were recruited to perform the required data acquisition. All drivers possessed a full "G" class driver's license in Ontario. Two of the drivers were male, and one was female, all were over the age of 20 years. All vehicle operators were familiar with Eco-driving concepts and drove the uninformed trips with the basic principles of efficient driving in mind. The driving task was split amongst all drivers in both the uninformed and informed driving states. No driver completed more than 10 consecutive trips at a time.

Data collection began at a minimum of $100 \mathrm{~m}$ prior to entering the first intersection in the trip to allow for the effects of the EcoDrive application on the initial intersection to be observed. The trips were terminated after clearing the last intersection in the connected corridor, though the GPS data collection was continuous throughout the entire testing loop sequence. The raw data obtained from the GPS unit located on the top rear end of the Van was downloaded twice per testing day, once at noon and again upon completion of the final trip of the day. The variables of interest were the data point ID number, the 
date and time, the latitude, the longitude and the elliptical height, which is the height of the GPS unit above sea level. All other variables were discarded.

The GPS coordinates were converted from degrees, minutes and seconds into decimal degrees, for ease of use in ArcMap. The longitudinal values had to be made negative given the city of Ottawa is west of the prime meridian [56]. The GPS files for each day were compiled into a single file. These formatted, daily files were then imported into ArcMap for further analysis.

\subsection{ArcMap Evaluation}

All shapefiles were imported using the geographic coordinate system of GCS North American 1983 The shapefiles are then projected into a more defined coordinate system for the precise location of Ottawa on the globe. As the globe is approximately a sphere, distortions will take place when trying to look at any location of the earth on a flat surface such as a map. The projection recommended by the Canada Centre for Mapping and Earth Observation is Universal Transverse Mercator (UTM). This projection system breaks up the 360 degrees of the globe into 60 zones spanning 6 degrees each, as show in Figure 9. The city of Ottawa falls into the UTM zone 18 [57]. Once imported, shape files were then projected into the NAD 1983 UTM Zone 18N coordinate system. 




Figure 9: UTM zones within Canada and the shape of a UTM zone [57].

The beginning of the base map was created in ArcMap using a roads segments layer obtained from the City of Ottawa's OpenData Datasets [58]. This layer was then modified to only include the segments of roads along the route under consideration. Points of interest were indicated on the map as a point. These points include all 12 connected intersections as well as the location 100m upstream from the first intersection entering the corridor from either direction.

Graduated buffers, at 7.5, 15, and 25 meters were created around these points to allow for identification of the closest GPS point on either side of the node to be identified when analyzing individual trips. The resulting finalized base map can be seen in Figure 5.

The files containing each day's second-by-second GPS information were added as a point layer to the ArcMap file. The latitudes and longitudes were converted to Cartesian coordinate point features. Meters were selected as the length unit for the creation of the X Y coordinates. The $\mathrm{Z}$ component, the point's elevation, was set to be the elliptical height 
in meters as obtained from the raw GPS data. The X Y coordinates were added to each original point feature in the attribute table as new variables.

Each testing day's data was converted into a continuous line by connecting each consecutive GPS point. These lines were then split into individual runs, and all line segments and points outside the routes were eliminated. The circular buffers were used to decide which GPS point to be used as the start point of the trips. The rings allowed for the identification of the closer of two points when lying on either side of the location marker, seen in Figure 10. At the other end of each trip, the GPS points were all chosen as the closest point after completely crossing the intersection marker to ensure the Van would have cleared the intersection prior to terminating the trip. Making use of the snapping feature in ArcMap, all trips began and ended precisely at the location of a GPS point.

This variable start and end line caused the distances travelled per trip to vary. To counteract this variability, the average trip speeds were used as the measure to evaluate changes in travel times. The 3D lengths of each line were produced in ArcMap to be used for comparison and validation of the trip lengths calculated using the individual data points. 


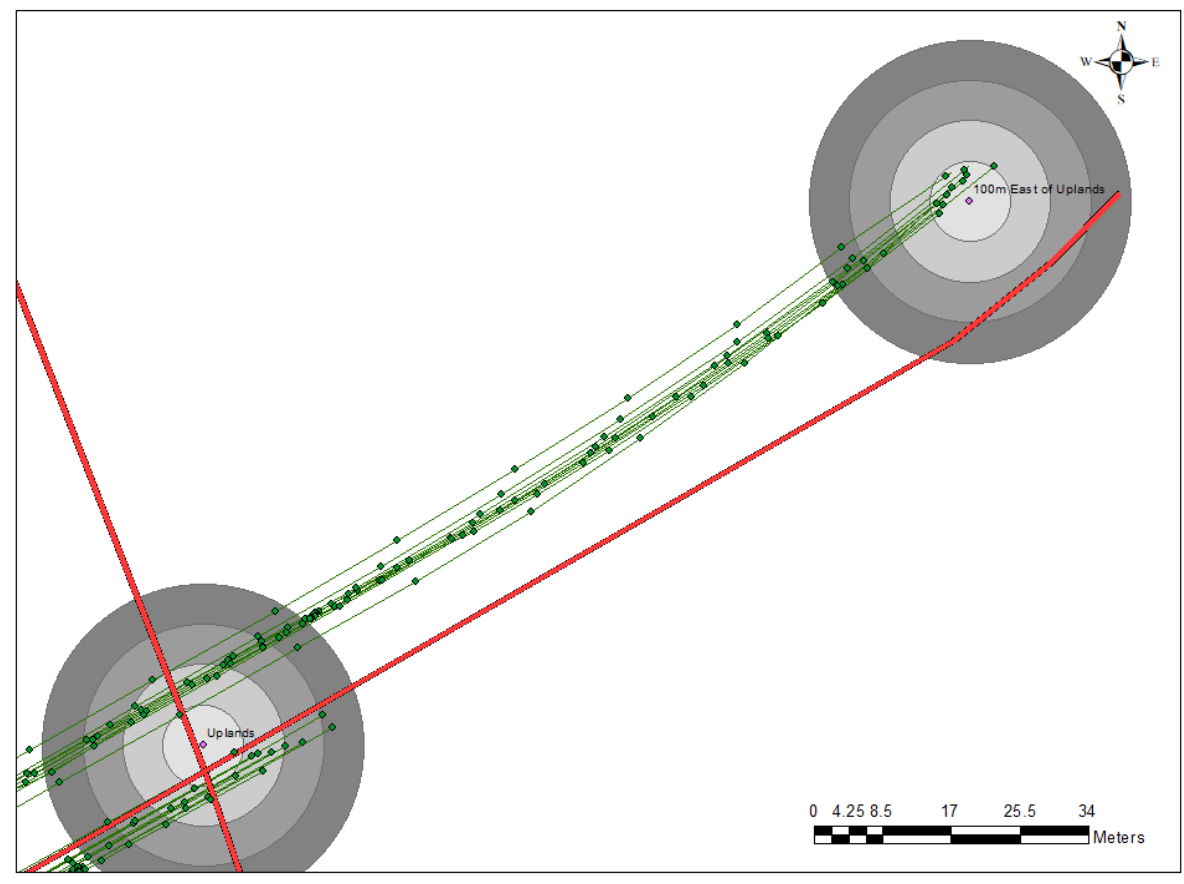

Figure 10: GIS Test run starting and termination points

\subsection{Cartesian Coordinate Based Calculations}

The GPS point feature files were exported from ArcMap to be analyzed in Microsoft Excel. Using the $\mathrm{X}$ and $\mathrm{Y}$ coordinates output by ArcMap, the horizontal distances from the previous point was calculated along with the change in elevation. These values were used to compute the road's grade at each GPS point. Calculating the grade of the road is essential to studying fuel economy and emissions because, as noted by Lebre et al. [43], a slight slope change along a route has significant impacts on results in the emissions analysis. Additionally, the slope of the road at individual points is essential in calculating the vehicle specific power which is noted as crucial for the emissions software used later in the analysis [59].

The average slope of each trip was also required as an input for the emissions modeling software. These calculations showed that overall the westbound runs had an average 
positive slope whereas the eastbound runs had an overall average negative slope. Both of these average slopes were well below $0.5 \%$. For each separate trip, the $3 \mathrm{D}$ distances were combined to calculate the total trip length.

The elapsed time between consecutive GPS readings were calculated to inform analysis. Although the GPS was set to take a recording at a one second resolution, there were times where the signal was lost causing the interval to be longer than a single second. The instantaneous speeds and accelerations were calculated for each GPS point, using the elapsed time between points. The trip length and speeds were converted to miles and miles per hour respectively for the inputs to the emissions estimation software.

\subsection{Stops}

The number of stops made in each trip was calculated based on the time spent idling. The first time stamped idle in a series was identified as new stops. The number of stops was calculated based off a new stop beginning a minimum of 50 meters at least from the previous stop. This distance was used to only account for stops that would have had the Van accelerating back up to a decent speed and thus consuming significant amounts of fuel. The distance of 50 meters was chosen to allow for the slight advances that can occur at intersections while waiting in the queue to not be observed as new stopping incidents.

These instances of stop-and-go movements do not reflect the usefulness of the EcoDrive application as such an event would take place only when traffic would have been a limiting factor. For this reason, the peak periods were avoided while testing the application. However, the corridor contains one of the five bridges outside of the 
downtown core which crosses the river, allowing east-to-west movement across the city. This causes the corridor to become congested occasionally during off-peak hours.

\subsection{Initial Data Screening}

\subsubsection{EcoDrive Malfunction}

Of all the trips conducted in the informed driver state, 18 were conducted with the application only displaying the remaining time in the current signal phase. Though the driver was receiving some additional information, the predictive SPaT information and the driving advice were absent. Since the EcoDrive application was not functioning to its full capacity during these trips, they were removed from the analysis. This decision was made prior to the completion of the testing period, which allowed for replacement trips to be conducted.

\subsubsection{Accident}

During one of the informed driver phase days of testing, an accident occurred in the lefthand lane on a westbound trip near the intersection of Antares Dr. and Lester St. This caused the closure of the westbound left turn lane. Police cars and ambulances were present requiring drivers to reduce their speed and move over as they passed the collision. On the third pass by the accident location, the vehicles had been removed, and the emergency workers were no longer on the scene. It was clear the previous two runs were conducted in significantly different traffic conditions from all other westbound runs. These trips were removed from the analysis because of this discrepancy. With the knowledge of the removal of these two trips, an additional two trips were added to the informed driver phase of testing. 
The uninformed driver state had an initial average speed of $41.25 \mathrm{~km} / \mathrm{h}$ while the combination of all informed driver states had an initial average speed of $37.55 \mathrm{~km} / \mathrm{h}$. Upon the removal of the 20 replaced trips, this average speed of the set of informed driver samples became $36.85 \mathrm{~km} / \mathrm{h}$.

\subsubsection{Lunch Hour}

The data collection plan outlined that testing was to be conducted on weekdays between the hours of 9:30 am and 3:30 pm, with the exception of the noon hour. Ten incidents were recorded within which portions of the trip occurred between the hours of 12:00 pm and 1:00 pm. This time is very unpredictable traffic wise, especially at certain intersections along the corridor. Trips that had spilled over into this hour were disproportionately present in the informed driver testing phase but were removed from both conditions.

\subsubsection{Heavy Traffic Events}

To compare the traffic conditions of various trips, ArcMap was used to display the locations where the Van's speed was unusually slower. Video footage of the identified slower periods was used to analyse the traffic conditions present during these trips. All testing days' GPS data points were overlaid on the same map. A graduated symbol was applied to the GPS points based on their speeds, showing only those which has speeds less than $25 \mathrm{~km} / \mathrm{h}$. The symbol was set to change gradually from green $(25 \mathrm{~km} / \mathrm{hr})$ to red $(0 \mathrm{~km} / \mathrm{hr})$. After the removal of the previously identified 20 trips, this visual allowed for areas to be identified where the Van was travelling unusually in comparison to all other trips made. The map with all slow points can be seen in Appendix C. 
As can be seen in Figure 11, where clusters of points are visible on the map represents the common places that the Van slowed or stopped. These clusters frequently occurred at intersections. Other areas can be seen where there are very few or only a single trip that was held up at a specific location. Potential outlier trips were identified where there were more than 20 consecutive points, representing approximately a 20 second delay, present outside of a cluster. The dates and times of these trips were recorded to be further analyzed using the video footage of Camera 1, which had been used to record traffic conditions throughout the testing.

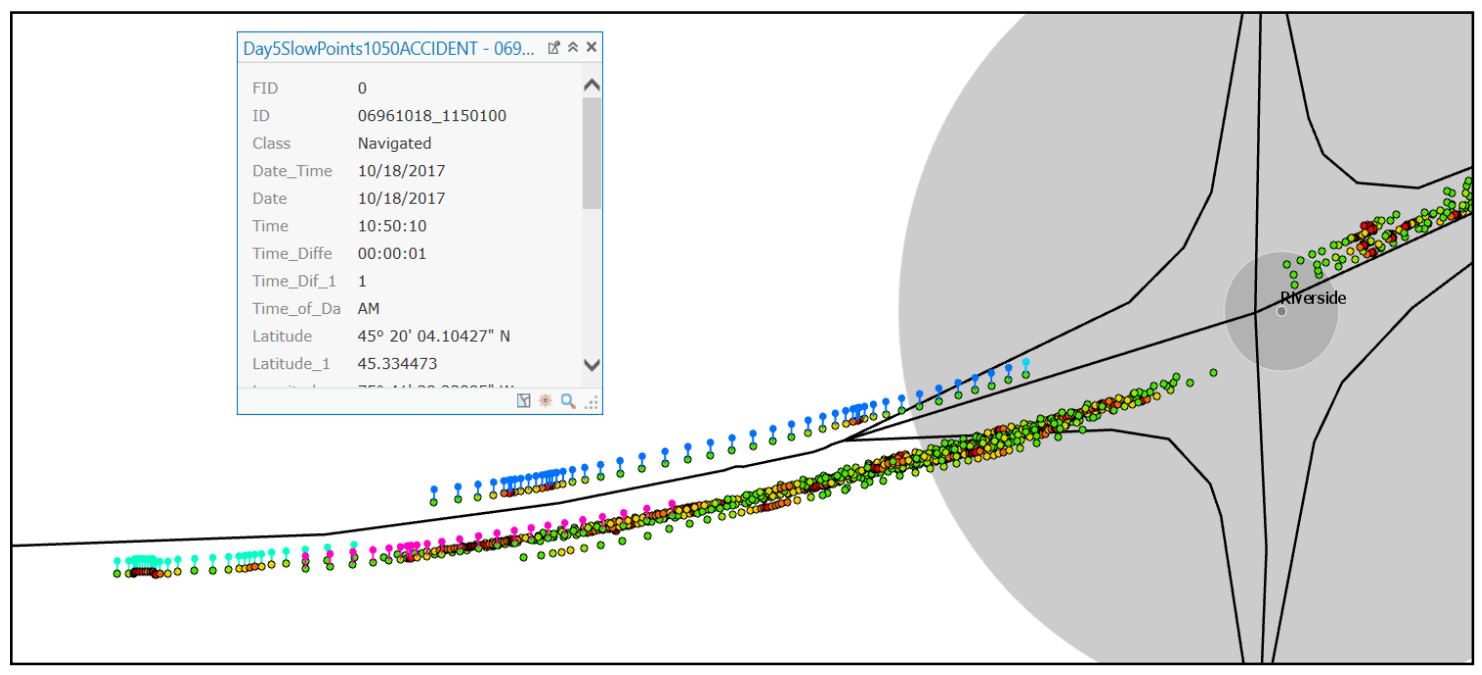

Figure 11: Outlier point identification

All of the groupings of points on the list of possible outlier trips were evaluated through review of the video footage. There were six trips removed as outliers from this set. Five of these trips were removed from the analysis when the video footage exposed that the Van had been significantly delayed by having to sit through an extra signal cycle at any given intersection. The traffic volumes at these times were deemed to be abnormally high as such events only took place five times over the course of the testing. 
A few of the identified potential outlier trips had queues at intersections that were longer than usual. This resulted in a trail of points outside of the cluster present at an intersection, such as can be seen by the trip represented in pink in Figure 11. The trips that were able to pass through the intersection in a single cycle, despite the abnormally long queues, were not deemed to be outliers as there was not a full signal cycle delay imposed on the Van.

The final trip removed as an outlier due to the traffic conditions was the third trip westbound following the accident. The vehicles involved and the emergency vehicles had cleared the scene of the accident which leads to the assumption that testing would no longer be affected. This run can be seen, represented by the blue markers, in Figure 11. The shockwave created by the accident had slowed traffic upstream of the accident. This was the only trip not previously identified as an outlier to have slow points on this stretch of road. 


\section{Chapter 7 Emissions Simulation Methodology}

\subsection{Motor Vehicle Emissions Simulation software (MOVES)}

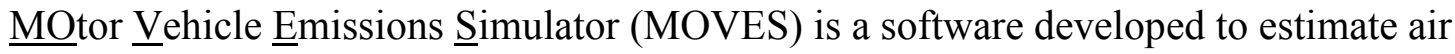
pollution emissions from mobile source designed by the US EPA Office of Transportation and Air Quality. CMEM, which was produced jointly by the National Cooperative Highway Research Program and the EPA and last updated in 2006, served as a foundational precursor for the development of MOVES [60]. This new simulator software is stated to be the EPA's best available tool for estimating GHG emissions and energy consumption by the transportation sector. It is currently the regulatory emission model for all states except California [61]. This state of the art model has been established based on the analysis of millions of emission tests. The initial model was created in 2010 and has been updated as the Agency's understanding of vehicle emissions has developed [62]. The most recent model at the time of this study was the 2014a model which had been updated in 2016 .

MOVES 2014a software has been designed to allow emissions modeling on multiple scales. The software provides the choice of three basic modeling scales. The macroscopic scale is intended to cover the national scale and can be used to develop national emissions inventories or to demonstrate the effects of large scale regulation changes. The mesoscopic scale is designed to model at the county level which can be used for regional transportation conformity evaluation and is the required methodology for the development of criteria pollutant inventories for State Implementation Plan across the US. The project scale is the model's microscopic level evaluation which can be used for 
modeling corridors, performing environmental impact assessments, or performing hot spot analysis [63] [64]. To best estimate emissions produced by the Van while travelling the HCRC project level analysis was used in the case of the EcoDrive evaluation.

The software generates a set of default values based off of initial inputs. The quality of the output is reliant on the quality of the input, as with any model. These defaults should be customized where possible to obtain more reliable results.

Of all the inputs vehicle fleet and activity data are the most sensitive inputs into the emissions estimation software as can be expected [59]. In the case of the HCRC, the fleet is known with confidence to be a single vehicle. The fleet activity data in MOVES is based on the VSP. VSP is an indicator of the ratio of the vehicle's output power to its mass. MOVES places high importance on the instantaneous engine power outputs which are key factors for this method of emissions modeling. As can be seen in Equation 2, the vehicle's instantaneous speed $(\mathrm{m} / \mathrm{s}), \mathrm{v}$, acceleration $\left(\mathrm{m} / \mathrm{s}^{2}\right), \mathrm{a}$, and the grade of the roadway (\%), s, are critical factors in the computation of VSP for each GPS point. The original VSP formula is a representation of engine load produced by acceleration, rolling resistance, aerodynamic drag along with the kinetic and potential energies of the vehicle over the vehicle's mass. A generic set of coefficients for estimating VSP of a typical light duty vehicle was used [64].

Equation 2 Vehicle Specific Power VSP $=v *[1.1 a+9.81 * S+0.132]+0.000302 * v^{3}[64]$

Previous modeling of emissions on a microscopic scale has shown that roadway grade has a significant effect on emissions [43]. The magnitudes of this effect have been empirically shown to be within a magnitude of significance be between $50 \%$ to $200 \%$ 
depending on the roadway grade and pollutant being modeled. A sensitivity analysis was performed on the MOVES model by Yao et al. [59]. This analysis concluded the VPS for light duty vehicles is most affected by speed while grade and acceleration have a lesser impact. All three of these factors were deemed to be crucial for MOVES Analysis. To properly use this model, it is essential to obtain and inventory of the vehicle's exact position and roadway grade in testing. The single second resolution positional data obtained from the GPS unit allowed for the calculation of the VSP at each point. All these factors allowed for accurate inputs of the fractions of time spent in various states which in turn developed greater confidence in the results produced by MOVES.

\subsection{MOVES Analysis}

With the one second resolution data, the MOVES 2014a Mobile Source Emissions Model was able to be used at a project level scale to analyze individual trip data. This model is used to estimate the GHG emissions and energy consumption of the Van on a microscopic scale for each trip. Every trip was run through MOVES and required the input of a specific set of operating conditions based on the way the Van had been driven on a second-by-second basis. With this software, knowledge of local environmental factors, detail of the Van's itself, and fuel used along with the one second resolution trip data it is possible to obtain estimations for the amount of fuel consumed as well as the amount of various emissions produced over the course of the individual trips.

\subsubsection{MOVES Run Setup}

The setup for each run of the MOVES software is done through the MOVES graphical user interface starting with the creation of a run specification or RunSpec. The RunSpec 
requires many inputs to be populated with parameter and choices. These initial inputs designate the requirements for the next stage which is the input of more detailed data of the specific scenario being model into the Project Data Manager. These inputs are stored in a database managed by MySQL, an open-sourced database management system, as required by the MOVES program. Each trip had its own MOVES RunSpec and subsequently its own input database. A new output database was created for each data testing phase to allow the results to be analyzed separately and compared to one another.

\subsubsection{RunSpec}

The MOVES RunSpec is used to define the general scenario in which each trip took place. It is in the RunSpec where the scale is defined for the level of precision in which the modeling will occur. These inputs define what will later be required by the Project Data Manager.

\subsubsection{Scale}

The analysis in MOVES was conducted at the Project scale which is the smallest of the three scale options, allowing for emissions simulations on a microscopic scale. The emissions rates and correction factors used in the Project Scale analysis are the same as those used in the National and County Scale analysis. The difference lies in the fact that at the project level the default MOVES growth, Vehicle Miles Traveled, and population values cannot be used, and the link definition and activity are required to be described to a considerably higher level of detail requiring second-by-second data on the movements of the vehicle [63]. The project scale represents the emissions estimations at the link level 
and produces the estimation in terms of the emissions emitted over the course of each trip individually. These values will then be used to calculate hourly averages for comparison.

\subsubsection{Time Span}

In the project level of analysis, each run can only consist of a single year, month day and hour. Therefore, trips were always entered into the RunSpec as the hour in which they began no matter how proportionally they were split amongst hours. In MOVES the days of the week are categorized as either Weekdays or Weekends. All of the test trips were conducted on weekdays.

\subsubsection{Geographic Bounds}

The Geographic Bounds input requires the choice of a United States County or a Custom Domain. Through exploration of nearby counties, the St. Lawrence Country in New York was chosen to be used as the geographic region to best represent the area in which the testing had taken place. The county borders Ontario, along the St. Lawrence River, between Mallorytown and Cornwall. St. Lawrence County's proximity to Ottawa along with the fact that it is bordering a river were the two main reasons for choosing this county as the base layer to be amended to match the EcoDrive project. The geographic comparison can be observed in Figure 12.

The majority of the defaults given by the chosen County in MOVES are modified within the Project Data Manager throughout the analysis. These modifications made to the defaults were significant, permitting this County to imitate Ottawa and the conditions present in the real world testing for the EcoDrive application. 


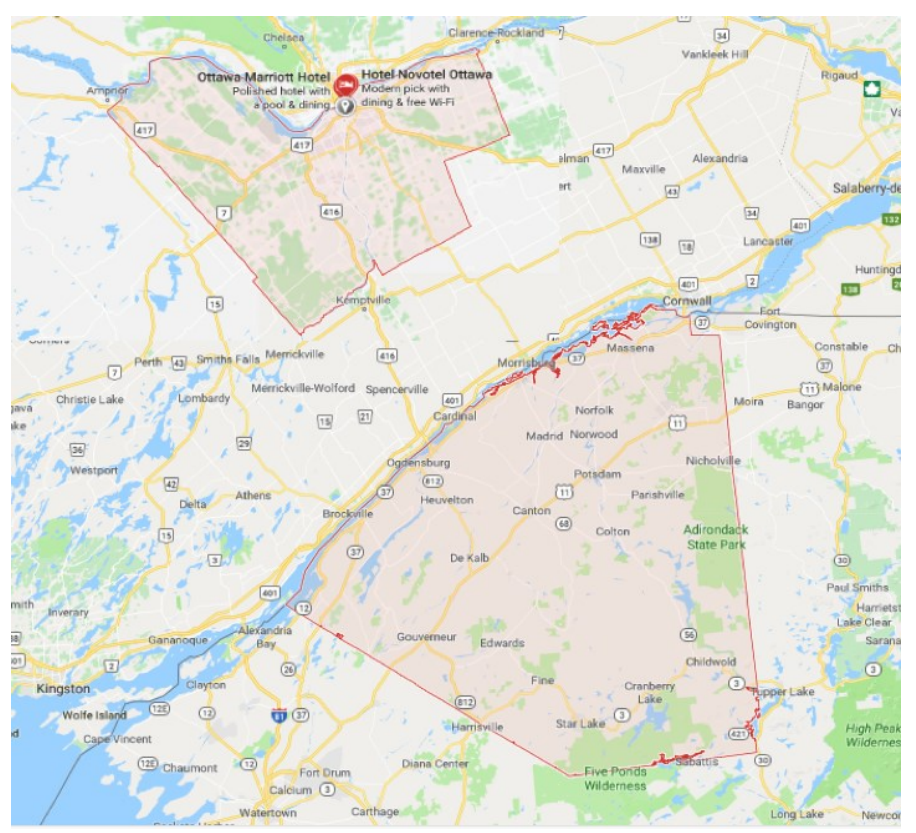

Figure 12: The Municipality of Ottawa (top) and St Lawrence County (bottom) maps

\subsubsection{Vehicle Equipment}

The only Vehicle Equipment input for the MOVES model, in the case of this study, is the Van. This study aims to only evaluate the emissions from this single vehicle as it is the only one operating on the road with the assistance of the EcoDrive IVU. There was not an option of a passenger van class in the list of available vehicle choices. The closest options were gasoline passenger car and gasoline passenger truck. Through information available from the EPA, noting that passenger vans are more massive than cars and tend towards being less fuel efficient, the vehicle class chosen for this model was of the class of a passenger truck fueled by gasoline.

\subsubsection{Road Type}

Similarly, only a single road type input was used in the case of this analysis. An Urban Unrestricted Access class road represents the corridor in the model. As there are no 
diversions from the main route, a single link was selected to represent the model. The link, in this case, was defined as the entire length of the HCRC on which testing took place with the exception of the lengths added by the U-turning movements. The corridor along this section is classified as an arterial roadway, in MOVES terminology, is defined as having unrestricted access.

\subsubsection{Pollutants and Processes}

This section allows for the selection of output pollutants. For certain pollutants to be calculated, other prerequisite pollutants were first required to be computed. The list of pollutants output, once the prerequisites were selected, was comprised of: HO2 (Aerosol), Composite - Non-Elemental Carbon PM, Sulfate Particulate, Elemental Carbon, Primary

Exhaust PM2.5, $\mathrm{CO}_{2}$ Equivalent, Fossil Fuel Energy Consumption, Total Energy Consumption, Atmospheric $\mathrm{CO}_{2}$, Volatile Organic Compounds, Non-Methane Hydrocarbons, Nitrogen Dioxide (NO2), Nitrogen Oxide (NO), Sulfur Dioxide (SO2), Ammonia (NH3), Nitrous Oxide (N2O), Methane (CH4), Oxide of Nitrogen (NOx), Carbon Monoxide (CO), and Total Gaseous Hydrocarbons. The main outputs of interest are the $\mathrm{CO}_{2}$ Equivalent and the Fossil Fuel Energy Consumption.

\subsubsection{Outputs}

The output units selected were the mass in grams, the energy units were Joules, and the distance units were Miles. These units were selected for consistency with required inputs for the MOVES software. This is also where the Output SQL database was selected, depending in which phase of testing the trip belonged. 


\subsubsection{Project Data Manager}

The population of the Project Data Manager with individual trip specific information, is the second preprocessing step required for the MOVES analysis. This section is typically filled with the information provided by the County selection, and thus many of the default categories required updating to replicate the scenario in which the testing took place. Five inputs that are unique to the project scale analysis are: Links, Off-network, Link source type, Operating mode distribution, and Link Drive schedules [63]. These and the rest of the inputs were able to be exported as an Excel file to be used as a template to be modified and reloaded into the software.

\subsubsection{I/M Programs}

This input pertains to the inspection and maintenance programs in place in the county or state selected. The State of New York most commonly conducts "Smog and Emissions Testing” annually through OBD testing of light diesel and gasoline fueled cars manufactured after 1996 [65]. This test ensures that certain parts of the vehicle are working properly so that the vehicle is not polluting excessively. A similar test takes

place in Ontario on vehicles that are older than six years of age [66]. This input needed to be modified to increase the testing time to biennial, and the compliance factor was increased to $100 \%$ as the testing is required for re-registration and the vehicle's registration was current.

\subsubsection{2. $\quad$ Operation Mode Distribution}

The operation mode distribution required the second-by-second GPS data to create the input. Each GPS point falls into one of the 23 operating mode bins. The first two bins are 
braking, which was defined as when the acceleration was less than $-1 \mathrm{~m} / \mathrm{s}^{2}$ or idling when the Van was not braking, and the speed was less than $5 \mathrm{~km} / \mathrm{h}$. The rest of the operating mode bins depended on a combination of the VSP and speed in MPH.

The VSP was calculated at each point using Equation 2. Each point's elapsed time was added to the operating mode bin to which it belonged. The run was then divided into the fraction of the total trip time spent in each speed bin. These fractions were inputted for each pollutant source to fill the operation mode distribution template.

\section{Operating Modes for Running Exhaust Emissions}

\begin{tabular}{|c|c|c|c|c|c|}
\hline & & Spe & Class & nph) & \\
\hline & & $1-25$ & $25-50$ & $50+$ & \\
\hline & $30+$ & 16 & 30 & 40 & \\
\hline & $27-30$ & & & & 21 modes representing \\
\hline Ф્ & $24-27$ & & 29 & 39 & $\begin{array}{l}\text { "cruise \& acceleration" } \\
(\mathrm{VSP}>0)\end{array}$ \\
\hline$\overline{\mathrm{c}}$ & 21-24 & & 28 & 38 & \\
\hline$\stackrel{ \pm}{\gtrless}$ & $18-21$ & & & & $\begin{array}{l}\text { PLUS } \\
2 \text { modes representing }\end{array}$ \\
\hline$\underline{z}$ & $15-18$ & & & 37 & "coasting" (VSP<=0) \\
\hline gn & $12-15$ & & 27 & & PLUS \\
\hline$\frac{\pi}{2}$ & $9-12$ & 15 & 25 & & One mode each for \\
\hline 0 & $6-9$ & 14 & 24 & 35 & decel/braking \\
\hline$\stackrel{\oplus}{\lessgtr}$ & $3-6$ & 13 & 23 & & Gives a total of \\
\hline & $0-3$ & 12 & 22 & 33 & $\begin{array}{l}\text { Gives a total of } \\
23 \text { opModes }\end{array}$ \\
\hline & $<0$ & 11 & 21 & & 7 \\
\hline
\end{tabular}

Figure 13: MOVES Operating Mode bins for light duty vehicles [67]

\subsubsection{3. $\quad$ Age Distribution}

This template provides the average spread of ages of the vehicles in the county selected. As the only vehicle being modeled is known to be the Van, this template was changed to have an input of 0.00 for all years other than the age category for a vehicle built in 2003 . The age fraction for a 14-year-old vehicle, the age of the Van, was set to 1 . 


\subsubsection{Fuel}

The Fuel inputs were separated into four files. The templates for these files show the average dispersion of fuel supply, the formulations of various fuel types, the fraction of use of the different fuel types and sub types, as well as the fraction of engines types across the county. With the use of a single vehicle these files needed to be narrowed to the exact fuel scenario present in the test Van. A single fuel supply was given the entire market share, as the Van only used one type of fuel. The fuel formulation was set to gasohol (E10) fuel [68]. The usage was set to the full usage to be this fuel. The engine in the Van is a model year 2003 conventional internal combustion which was reflected in the final fuel input file.

\subsubsection{Meteorology Data}

The meteorology input was customized for each trip being considered. The month, hour, the relative humidity and the temperature were placed in the template. Hourly relative humidity and temperature were obtained from the Canadian governmental archives [69]. These temperatures were then converted to Fahrenheit to comply with the MOVES input requirements. To follow the same pattern as the time the environmental inputs were set to align with the hour in which the test run began.

\subsubsection{6. $\quad$ Link Source Type}

The link source type required the link to be given an identifier. As there was only one road travelled each trip only one link was used. It was given the identifying number 1, and the entire associated usage fraction was attributed to this link. 


\subsubsection{Links}

The link inputs were also customized for each trip's MOVES run. This input required the link identifier, the trip length, in miles, the average speed, in miles per hour, and the average grade of the trip. The link was given a traffic volume of one so that only the outputs of the single vehicle would be calculated.

\subsubsection{Unchanged Inputs}

In the case of these MOVES runs, there was no hoteling as this study does not deal with commercial trucks. As the period of time was a short window of an hour on a specific day, the data was not required to be retrofitted. The network of concern only consists of on road travel; therefore, the Off-Network inputs were not required. As the trips being modeled were of a specific length, and the single vehicle trajectories were known, the Link drive cycle input was not used. 


\section{Chapter 8 Evaluation of EcoDrive Technology}

The two databases, one for each driver operating condition (uninformed and informed), containing the output from the MOVES software were analyzed and compared to one another. The data initially output by MOVES had been formatted to represent the amount of emissions produced per simulation run which, in the case of this evaluation, is a single trip on the HCRC. The carbon dioxide equivalents were converted to the more common metric of the rate produced in $\mathrm{kg} / \mathrm{hr}$ for each MOVES run. The fossil fuel energy consumption was converted from Joules per trip to the extrapolated consumption rate in $\mathrm{L} / \mathrm{h}$. This conversion was done with the use of the factor obtained from Statistics Canada's Energy Statistics Handbook for gasoline equating to $35 \mathrm{MJ}$ of energy/L [70].

\subsection{Final Outlier Analysis}

The trip information and MOVES Emission outputs were imported into R software version 3.4.3 for further analysis. The outliers identified previously were removed from the data sets prior to being extensively analyzed as their reasons for being excluded were external from the statistical analysis. The principal variables for evaluation are $\mathrm{CO}_{2}$ equivalent emissions, fossil fuel energy consumption, and average trips speed.

Boxplots were created in $\mathrm{R}$ to investigate the presence of any statistical outliers in the remaining dataset. The outliers are identified as being outside of the whiskers located at +/-1.5 interquartile ranges on either side of the first and third quarter. Individual plots were created to investigate average speed outliers, $\mathrm{CO}_{2}$ equivalent outliers, and fossil fuel consumption outliers. These plots, along with all other R outputs, can be seen in Appendix F. 
The average trip speed plot identified no outliers for the uninformed driver phase of testing, but the three lowest average speed trips from the informed driver phase were identified as outliers. Through video analysis, two of the identified trips, informed driver Run 8 and Run 10, were noted to be traveling westbound while the aforementioned accident was still present in the eastbound lanes. These two trips were removed as the collision and presence of emergency vehicles could have had an adverse effect on the opposing traffic flows as well. The third outlier was Run 28 of the informed driver testing. There is no video data for this run to be verified due to a technical malfunction and this run had not been previously identified in the analysis of unusual slow points. As reasoning was not available to discount this run it remained a part of the analysis.

The outliers for the average $\mathrm{CO}_{2}$ equivalent emissions and average fossil fuel energy consumption were the same three trips. These trips included the fastest trips in both phases of testing. Run 8 from the informed driver phase was an outlier for all three variables evaluated and was also the lowest speed run overall. This run has already been removed in the previous variable outlier analysis. The other two identified trips were Run 13 of the uninformed driver phase and Run 4 of the informed driver phase. These identified outliers were reviewed by means of video footage and no irregularities were observed. These runs were also kept as a part of the analysis.

The final data sets consisted of 46 trips made without the use of the EcoDrive IVU while only 38 trips remained in the informed driver phase set of samples. The uninformed driver phase set of samples had an even number of runs remaining in both directions while the informed driver phase ended up with two more runs conducted in the 
westbound direction. The average slope in this direction is less than $0.5 \%$. Therefore, it was assumed the extra two runs conducted in the westbound direction should not significantly affect the overall results.

\subsection{Significance Testing of the Core Variables}

In order to test the significance of the comparison, the distribution of the data first needs to be determined. Summary statistics were drawn from both the uninformed and the informed datasets. These summaries are available in Appendix F. Depending on the distribution of the data, the unpaired two-samples test used to evaluate significance differed. For a variable with a normal distribution, the T-test was used. Distributions that proved not to be normal were compared using the non-parametric Wilcoxon rank sum test. The core variables under consideration were average trip speed, fossil fuel consumption, and carbon dioxide equivalent emissions produced. The fraction of times spent in each speed bin (grouped speed ranges) and the number of stops made per trip were also evaluated for significance.

The Shapiro-Wilk normality test was first applied to all variables for both the uninformed and informed driver sample sets. This test determined if the data follows a Gaussian distribution, allowing for the T-test to be applied. Normally distributed data has a p-value from the Shapiro-Wilk test of less than 0.1 [71]. In the event that one distribution in a pair of informed and uninformed state variables was shown to not be normally distributed the Wilcoxon Ranked sum test was applied. The two-paired tests were initially run with the null hypothesis of the means or medians being the same. 


\subsubsection{Travel Time Analysis}

Of the core variables being evaluated, all were found to have normal distributions. The number of samples in both phases of testing with outliers removed was still above the minimum acceptable sample size of 31 . This minimum number of samples required had been determined by the CTL to be sufficient to produce statistical significance for the average trip speeds. The initial t-test of the average speeds produced a p-value above 0.05 indicating that the null hypothesis of the means being equal could not be rejected. The average speed for the uninformed driver phase was $41.44 \mathrm{~km} / \mathrm{h}$ while the informed driver phase was $39.70 \mathrm{~km} / \mathrm{h}$, with standard deviations of 4.72 and 4.2 respectively. These average speeds fell within the tolerance level of $1.75 \mathrm{~km} / \mathrm{h}$ set when calculating the required number of samples. A $-4.18 \%$ change was observed when examining the difference from uninformed to the informed driver states suggesting that the mean travel speed for the uninformed driver was faster.

The statistical significance was tested a second time using the t-test with the null hypothesis that the uninformed driving phase had an average speed of less than that recorded in the informed driver phase. This test produced a p-value of 0.0397 and thus statistical significance at a 95\% confidence level. This result allowed for the rejection of the null hypothesis, inferring that the average speeds of trips on the HCRC is not significantly reduced by the use of the EcoDrive application.

Travel time is important to road users, with many models attempting to put a dollar value to it for cost benefit and mode choice analysis. To introduce such a technology to the public or to industry, where time has a monetary value, the effects on travel time need to 
be thoroughly evaluated. As its been shown, the use of the EcoDrive application did not cause the average speeds to be lower when comparing the uninformed driver to the informed driver. As distances varied for trips conducted on the HCRC, the average speed was used to infer travel times. Therefore, travel times with the use of the EcoDrive application are not any longer than trips conducted without the application. These findings are only impactful when paired with results for fuel consumption and emissions.

\subsubsection{Fuel Consumption and Emissions Analysis}

The reduction both in average fossil fuel energy consumed and $\mathrm{CO}_{2}$ equivalent produced proved to be statistically significant when comparing the informed driver state samples to the uninformed driver state samples, as seen in Table 2. These results allowed for the rejection of the null hypothesis of the informed and uninformed driver state distributions having equal means. With the use of the EcoDrive IVU drivers were able to obtain a 9.64\% savings in average fuel consumption and $9.62 \%$ percent reduction in GHG emissions production. Having realized both fuel savings and emissions reductions, the analysis clearly indicates that the use of this kind of vehicle to infrastructure connectivity can provide economic and environmental benefits to users in real world scenarios. The reasons for these savings are investigated through the secondary variables.

Table 2: Test of differences results for Average speed, GHG emissions and fuel consumption

\begin{tabular}{|c|c|c|c|c|c|c|c|c|c|c|}
\hline \multirow[b]{2}{*}{ Variable } & \multicolumn{4}{|c|}{ Uninformed } & \multicolumn{4}{|c|}{ Informed } & \multirow[b]{2}{*}{$\begin{array}{c}\text { Percent Change } \\
\text { (uninformed to } \\
\text { informed) }\end{array}$} & \multirow[b]{2}{*}{$\begin{array}{c}\text { T-test } \\
\text { p-value }\end{array}$} \\
\hline & $\begin{array}{l}\text { Shapiro- } \\
\text { Wilks } \\
\text { p-value }\end{array}$ & Mean & $\begin{array}{l}\text { Standard } \\
\text { Error of } \\
\text { the Mean }\end{array}$ & $\begin{array}{l}\text { Standard } \\
\text { Deviation }\end{array}$ & $\begin{array}{l}\text { Shapiro- } \\
\text { Wilks } \\
\text { p-value }\end{array}$ & Mean & $\begin{array}{l}\text { Standard } \\
\text { Error of } \\
\text { the Mean }\end{array}$ & $\begin{array}{l}\text { Standard } \\
\text { Deviation }\end{array}$ & & \\
\hline Speed $(\mathrm{km} / \mathrm{h})$ & 0.37 & 41.44 & 0.70 & 4.73 & 0.38 & 39.71 & 0.68 & 4.20 & -4.18 & 0.07941 \\
\hline CO2E (kg/h) & 0.24 & 13.94 & 0.17 & 1.15 & 0.68 & 12.60 & 0.12 & 0.72 & -9.62 & $6.43 E-09$ \\
\hline Fuel (L/h) & 0.24 & 5.20 & 0.06 & 0.43 & 0.68 & 4.70 & 0.04 & 0.27 & -9.64 & $6.43 \mathrm{E}-09$ \\
\hline \multicolumn{9}{|c|}{ Ho=Average Speed in the informed state $<$ Average speed in the uninformed state } & & 3.97E-02 \\
\hline
\end{tabular}


Additionally, 95\% confidence intervals were produced using bootstrapping in R. These computed the difference in means of 9999 random samples taken with replacement. The confidence interval for the fuel consumption was a savings between $0.38 \mathrm{~L} / \mathrm{h}$ to a 0.65 $\mathrm{L} / \mathrm{h}$, consistent with the difference in means of a decrease of $0.5 \mathrm{~L} / \mathrm{h}$ seen in the study. The study mean difference was a decrease of $1.34 \mathrm{~kg} / \mathrm{h}$ in $\mathrm{CO}_{2}$ equivalent emissions which was central in the confidence interval of a $-1.02 \mathrm{~kg} / \mathrm{h}$ to $\mathrm{a}-1.74 \mathrm{~kg} / \mathrm{h}$ produced by the bootstrapping method. The histograms produced by these methods can be seen in Figure 14 and Figure 15.

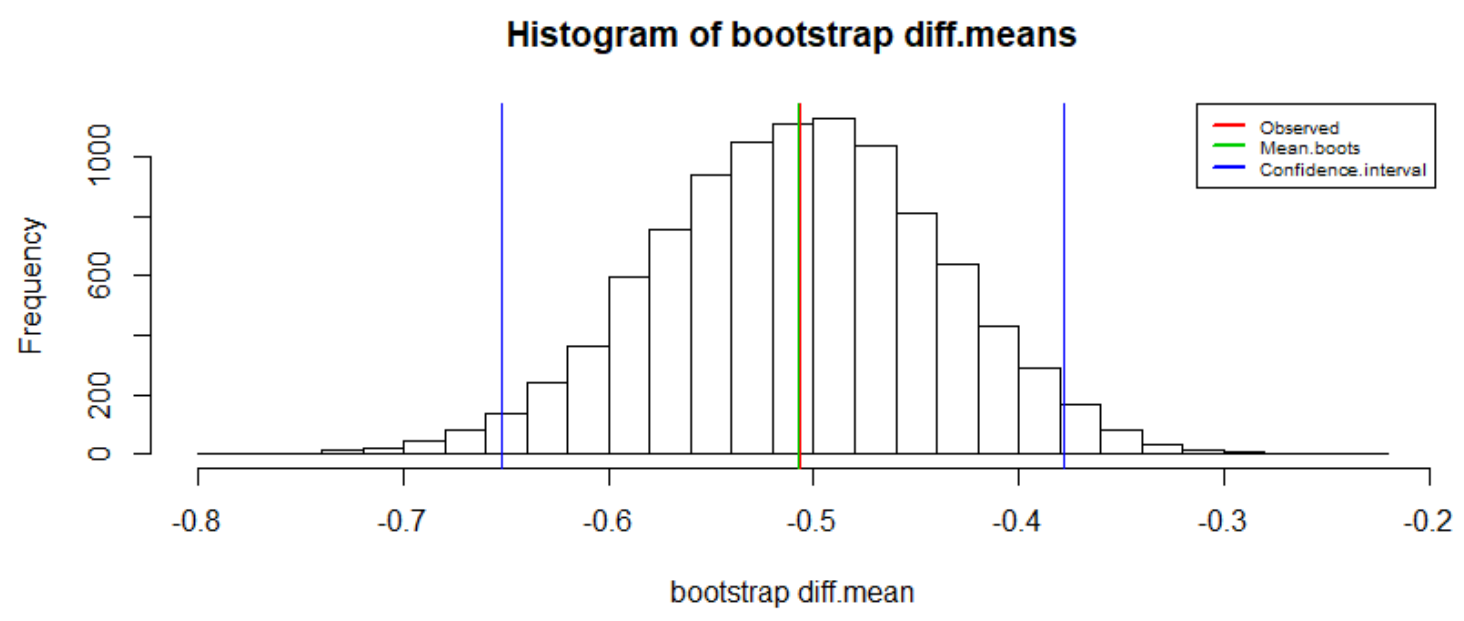

Figure 14: Histogram of bootstrap difference in fuel consumption and its confidence interval 


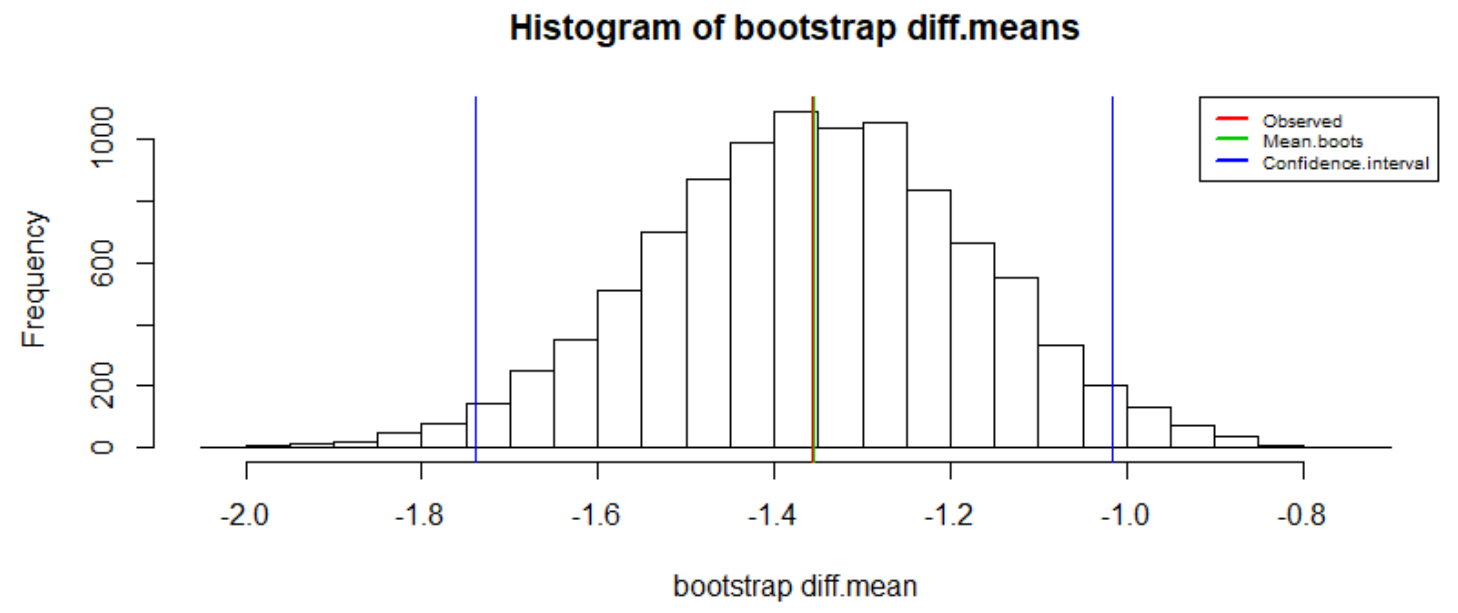

Figure 15: Histogram of bootstrap difference in $\mathrm{CO}_{2} \mathrm{E}$ and its confidence interval

\subsubsection{Secondary Factors}

Further statistical significance testing was performed on the fraction of time spent in all other speed bins used by the MOVES Operation Mode and Distribution input as well as the average number of stops made per trip. Of all secondary variables tested, four bins provided results with statistical significance at the required $95 \%$ confidence level. These variables with significance were the fractions of time spent braking, the fractions of time spent at low speeds coasting, the fractions of time spent at moderate speeds coasting and the fractions of time spent traveling between the speeds of $40 \mathrm{~km} / \mathrm{h}$ and $80 \mathrm{~km} / \mathrm{h}$ with a VSP between 9 and 12. The results for all secondary variables can be seen in Table 4 .

The amount of time spent in the moderate speed bin with a VSP between 9 and 12 was reduced $12.66 \%$. The other bins in the moderate speed category with high VSP also saw similar decreases, though not significantly. These reductions could be attributed to less hard accelerations taking place with the use of the EcoDrive IVU. 
Both of the fractions of time spent coasting increased while the fraction of time spent braking decreased from the uninformed to the informed driver states. These results can be attributed to messages received in the second, third and fourth scenario as described by the EcoDrive application.

In the second and fourth scenarios, a symbol communicated to drivers alerted them that they were not able to cross the upcoming intersection through either a white rectangle with an " $\mathrm{X}$ " at the center or a time to green surrounded by a red circle. These messages allowed the driver to confidently release the gas pedal to coast towards the intersection or reduce their speed gradually until coasting towards the intersection. These messages would appear shortly after the Van had crossed the previous intersection. The distance to the next intersection was anywhere from approximately $190 \mathrm{~m}$ to $1200 \mathrm{~m}$, as seen in Table 3. This advanced notice made it possible for drivers to react to the situation in advance of what may have been possible for them to perceive from the signals alone. At times these signals would be over a hill or around a bend, out of the line of sight of the driver. The fraction of time spent coasting at low speeds increasing by $31.45 \%$ while at medium speeds the fraction of coasting time increased by $21.45 \%$ with the use of the EcoDrive application. 
Table 3: Points of interest and distances along the route

\begin{tabular}{|c|c|}
\hline Cross Street & $\begin{array}{l}\text { Approximate distance from the previous } \\
\text { intersection (m) }\end{array}$ \\
\hline \multicolumn{2}{|l|}{ Uplands Drive } \\
\hline Paul Anka Drive & 400 \\
\hline Paul Benoit Driveway & 1200 \\
\hline Hunt Club Market & 450 \\
\hline Riverside Drive & 190 \\
\hline Prince of Wales Drive & 550 \\
\hline Antares Drive / Laser Street & 400 \\
\hline$\# 8$ retail & 900 \\
\hline Roydon Place and Sunderland Street & 500 \\
\hline Merivale Road & 400 \\
\hline Nepean Crossroads Centre & 170 \\
\hline Cleopatra Drive & 190 \\
\hline Average & 486 \\
\hline Median & 400 \\
\hline
\end{tabular}

Note: These distances do not include the spans of the intersections

In the uninformed driver state, drivers are more likely to maintain their speed up to the point where it is visible from the traffic signal that they will not be able to cross in the current green phase. Here the driver would be required to brake harder and possibly longer than they would have had they coasted towards the intersection, as they would have been traveling at a higher speeds. Overall the fraction of time spent braking saw a $17.33 \%$ decrease with the use of the EcoDrive application. The increase in coasting and 
decrease in time spent braking suggests that the application influenced driver behaviour to be more fuel efficient, resulting in the reduction in GHG emissions discussed above.

The third scenario identified by the EcoDrive application was highlighted as having the potential to create substantial savings by reducing the number of stops made. In the informed driver state this scenario was to provide the driver with a GLOSA recommendation below the speed limit, allowing them to cross the upcoming intersection without stopping. This recommendation should have allowed the Van to arrive at the intersection after the signal had changed to green and the queue had dissipated. Contrary to the predictions made by TTS, this scenario did not significantly decrease the average number of stops made along the corridor. The average number of stops with the use of the of EcoDrive IVU saw only a $0 . \%$ decrease without statistical significance. Similar to the average speed, this value was tested to see if the reduction was significant using an alternative null hypothesis. Those tests also resulted in no significance.

The recommendation for the third scenario relies on a predetermined queue length which in reality may have been longer than anticipated, therefore taking more than the few seconds allotted in the algorithm for it to dissipate. Should this be the case, the driver would arrive as the light had changed but the queue would still prevent the driver from continuing through the intersection. This would cause the vehicle to stop and idle behind the queued vehicles. This scenario does present the advantage that the GLOSA recommendation which the driver would have had, which would have been below the posted speed limit. As the Van approached the queue of vehicles waiting to cross the 
intersection they would be required to stop, but wouldn't have been traveling at the same higher cruising speed they would have likely been driving in the uninformed state.

As the number of stops did not significantly change throughout the testing, it is understandable that the amount of time spent idling was also not significantly altered. The fraction of the average trip times spent idling increased by $0.75 \%$ with the use of the EcoDrive application. As with the number of stops, this reduction was not proven to be a statistically significant change. 
Table 4: Secondary variable statistics

\begin{tabular}{|c|c|c|c|c|c|c|c|c|c|}
\hline \multirow[b]{2}{*}{ Variables } & \multicolumn{3}{|c|}{ Uninformed } & \multicolumn{3}{|c|}{ Informed } & \multirow[b]{2}{*}{$\begin{array}{l}\text { Percent } \\
\text { Change }\end{array}$} & \multirow[b]{2}{*}{$\begin{array}{c}\text { T-test } \\
\mathrm{p} \text {-value }\end{array}$} & \multirow[b]{2}{*}{$\begin{array}{l}\text { Wilcoxon } \\
\text { p-value }\end{array}$} \\
\hline & $\begin{array}{c}\text { Shapiro- } \\
\text { Wilk's } \\
\text { p-value }\end{array}$ & Mean & Median & $\begin{array}{c}\text { Shapiro- } \\
\text { Wilk's } \\
\text { p-value }\end{array}$ & Mean & Median & & & \\
\hline Stops & 0.00599 & 4.15217 & 4.00 & 0.064888 & 4.13158 & 4.00 & -0.50 & 0.94658 & \\
\hline Idling & 0.96284 & 0.19215 & 0.20 & 0.561821 & 0.19359 & 0.20924 & 0.75 & & 0.8963205 \\
\hline Braking & 0.53713 & 0.08242 & 0.09 & $1.00 E-06$ & 0.06814 & 0.06172 & -17.33 & & 0.000421 \\
\hline Coasting (under25MPH) & 0.00217 & 0.04139 & 0.04 & 0.05528 & 0.05441 & 0.05207 & 31.45 & 0.0221 & \\
\hline 0 to 3 & 7.45E-06 & 0.03104 & 0.03 & 0.012534 & 0.03768 & 0.03562 & 21.39 & 0.1365 & \\
\hline 3 to 6 & 0.00409 & 0.01821 & 0.02 & $1.73 E-05$ & 0.01698 & 0.01402 & -6.78 & 0.58861 & \\
\hline 6 to 9 & 0.345 & 0.02932 & 0.03 & $2.01 E+12$ & 0.02658 & 0.02477 & -9.36 & & 0.2608932 \\
\hline 9 to 12 & 0.00656 & 0.02377 & 0.02 & $8.11 E+13$ & 0.02634 & 0.02472 & 10.81 & 0.23856 & \\
\hline above 12 & 0.00577 & 0.01855 & 0.02 & $2.38 \mathrm{E}+13$ & 0.01566 & 0.01558 & -15.61 & 0.27311 & \\
\hline Coasting (25 to 50MPH) & 0.44453 & 0.1111 & 0.11 & 0.26049 & 0.13493 & 0.13615 & 21.45 & & 0.000945 \\
\hline 0 to 3 & 0.5816 & 0.09594 & 0.10 & 0.250111 & 0.10826 & 0.09935 & 12.84 & & 0.1304534 \\
\hline 3 to 6 & 0.2749 & 0.08753 & 0.08 & 0.346663 & 0.08764 & 0.08574 & 0.13 & & 0.882118 \\
\hline 6 to 9 & 0.65282 & 0.08616 & 0.09 & 0.057789 & 0.07744 & 0.07197 & -10.13 & & 0.1381148 \\
\hline 9 to 12 & 0.60239 & 0.08105 & 0.08 & 0.35447 & 0.07079 & 0.06807 & -12.66 & & 0.018212 \\
\hline 12 to 18 & 0.89599 & 0.07105 & 0.07 & 0.991499 & 0.062 & 0.06342 & -12.73 & & 0.18901 \\
\hline 18 to 24 & 4.52E-05 & 0.0125 & 0.01 & 0.000144 & 0.00901 & 0.00663 & -27.97 & 0.15922 & \\
\hline 24 to 30 & 8.35E-10 & 0.00149 & 0 & $7.92 \mathrm{E}-08$ & 0.00087 & 0 & -41.46 & 0.18167 & \\
\hline over30 & 5.07E-12 & 0.00077 & 0 & $8.22 \mathrm{E}-10$ & 0.00103 & 0 & 34.28 & 0.57712 & \\
\hline over 50MPH & 1.33E-06 & 0.00722 & 0 & $1.57 \mathrm{E}-08$ & 0.00382 & 0 & -47.11 & 0.05598 & \\
\hline 6 to 12 & 1.85E-09 & 0.00563 & 0 & $5.32 \mathrm{E}-10$ & 0.00273 & 0 & -51.53 & 0.09687 & \\
\hline 12 to 18 & $1.74 \mathrm{E}-10$ & 0.0024 & 0 & $3.04 \mathrm{E}-10$ & 0.00183 & 0 & -23.96 & 0.56892 & \\
\hline 18 to 24 & $2.55 \mathrm{E}-13$ & 0.00023 & 0 & $8.96 \mathrm{E}-13$ & 0.00013 & 0 & -43.86 & 0.50574 & \\
\hline 24 to 30 & $5.38 \mathrm{E}-15$ & 4.40E-05 & 0 & $1.61 \mathrm{E}-13$ & 4.70E-05 & 0 & 8.21 & 0.95572 & \\
\hline above30 & $5.38 \mathrm{E}-15$ & 3.50E-05 & 0 & $8.96 \mathrm{E}-13$ & 0.00013 & 0 & 266.65 & 0.34128 & \\
\hline
\end{tabular}

\subsection{Trajectory comparison}

A comparison was made of Run 21 of the uninformed driver testing and Run 39 of the informed driver testing for comparison of the speed profiles. Both trips occurred at around 9:30 am and were travelling in the westbound direction. The uninformed driver had an average speed of $46.67 \mathrm{~km} / \mathrm{h}$ while the informed driver run had an average speed of $46.57 \mathrm{~km} / \mathrm{h}$.

The trajectory for the driver receiving advice from the EcoDrive application, symbolized by the green line in Figure 16, displays steady speed throughout the majority of the trip. 
Over the course of the trip, the Van was made to stop only twice out of the possible 12 intersections which could have interrupted its flow. Alternatively, the uninformed driver trajectory, represented by the red line, sees five instances where its speed wavers visibly, and the Van was made to stop three times. It can be seen at around a minute and a half into the uninformed trip that the Van accelerates towards an intersection, seemingly in the dilemma zone, only to have to brake harshly to avoid a red light infraction.

The fuel consumed and emissions produced by these runs varies a great deal. The trip without the use of the EcoDrive application has a rate of production of $\mathrm{CO}_{2}$ equivalent of $14.26 \mathrm{~kg} / \mathrm{h}$ and a fuel consumption rate of $5.32 \mathrm{~L} / \mathrm{h}$. Alternatively, the informed driver trip has a rate of production of $12.33 \mathrm{~kg}$ of $\mathrm{CO}_{2}$ equivalent per hour and a fuel consumption rate of $4.6 \mathrm{~L} / \mathrm{h}$. A fuel savings and emissions reduction of $13.53 \%$ was observed in the trip where the informed driver made fewer stops and had a steadier speed profile. 


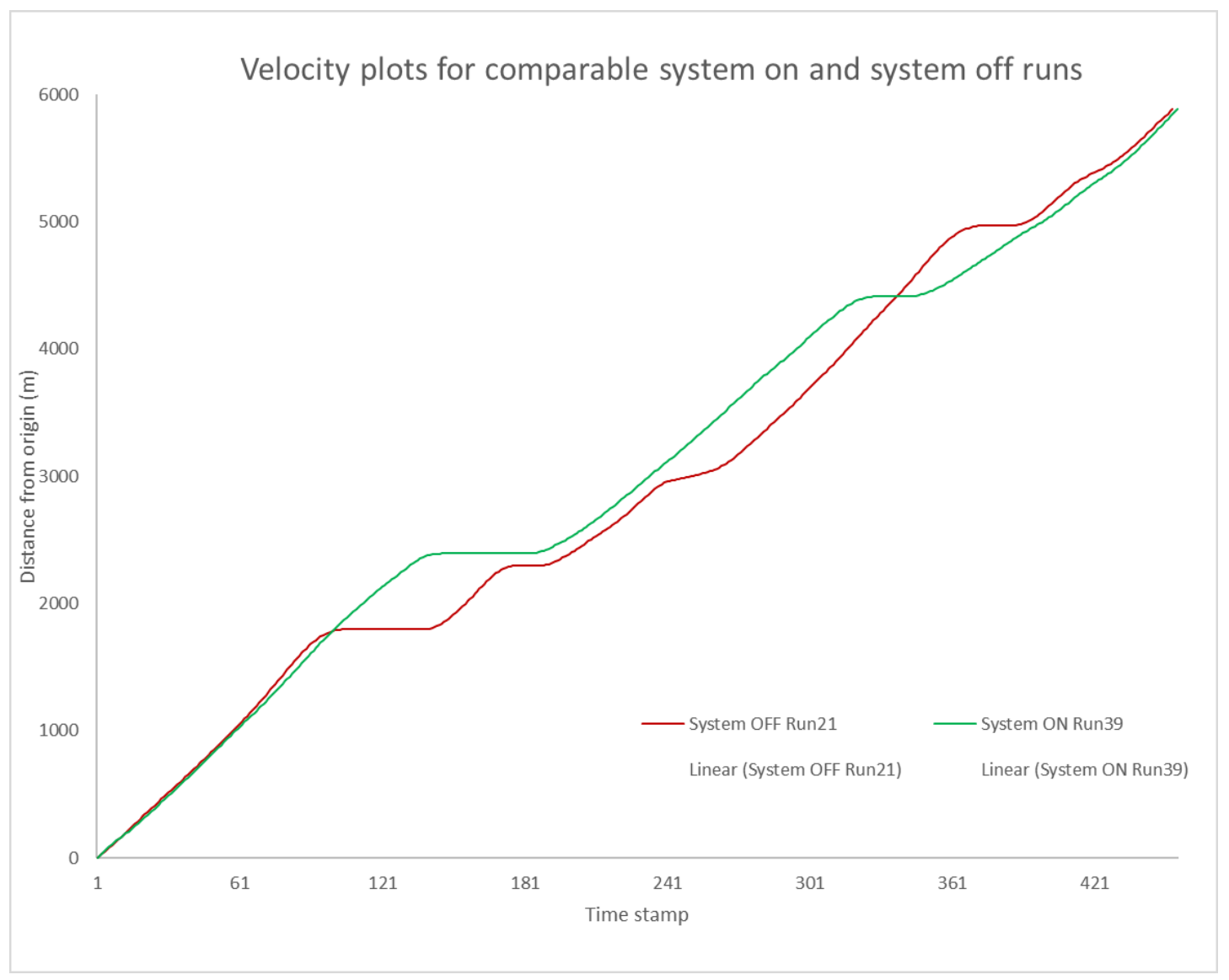

Figure 16: Comparison of uninformed and informed driver trajectories

\subsection{Evaluation of Results}

Through the simulations viewed in the literature review it was observed that vehicles using connected vehicle technology can enjoy a range of benefits in terms of fuel consumption, emission reductions, and travel time. Simulations are known to have limitations on their definitions of scenarios and parameters which do not always reflect reality. The field testing studies that have taken place in the field using velocity planning connected vehicle technologies also have limitations. The limitations are observing a vehicle uninhibited by traffic on a single lane road vehicle without the presence of multiple traffic signals in succession. These models have made substantial efforts to 
account for their shortcomings through study design, producing reliable results for specific scenarios. Previously predicted time savings on a single vehicle was seen in the reviewed literature as being between $1 \%$ and $7 \%$ with a real-world pilot observing a three-minute time savings along a $4 \mathrm{~km}$ longer corridor. These findings are not consistent with what was observed in this real-world testing, though the specific algorithms could be prioritizing different variables. In the case of the EcoDrive application, the average speeds were not significantly decreased with the use of the application concluding that there was no significant effect on travel times, positive or negative.

The range for fuel savings and emissions reductions observed in simulation and field testing of similar velocity planning algorithms ranged from $4 \%$ to $19 \%$ on individual vehicles. These values are consistent with what was observed in the EcoDrive pilot. With the use of the EcoDrive connected technology, a reduction in fuel consumption of $9.64 \%$ was observed while GHG emissions were reduced by $9.62 \%$. These savings can be partially attributed to the increased amount of time spent coasting and the reduced amount of time braking.

Many of the previously mentioned studies only offer substantial benefits at a certain market penetration. With the introduction of more equipped vehicles, velocity planning systems could become more accurate at predicting queue lengths and could potentially reduce the number of stops made to produce even more significant benefits. The addition of more technology certainly has the potential to increase the benefits of any connected velocity planning tool. However, the savings produced by the EcoDrive application are 
substantial and are readily available to any driver who chooses to follow the recommendations.

This study did not evaluate driver compliance to the application. The compliance could be greatly affected by traffic, as complying with the speed recommendations may not be possible when traffic is at a level where lane changes are not freely available. This is a reality which will face all drivers using the application, and thus it was not deemed to be critical to the study. Real world implementation of this application may see lowered results should the driver have other priorities at the time of use that would affect compliance. In other circumstances, driver lack of compliance may be seen when the application is not giving correct advice. Throughout testing, there were a few instances where the advice given did not match the current signal phase.

Future analysis should be conducted to include driver compliance and reasoning for noncompliance. Furthermore, an increase in the level of certainty used in the algorithm would improve the reliability of messages received. This reliability will become especially important if this technology moves into levels of automation where the driver is no longer in control of the longitudinal functions of the vehicle, which can already be observed in the case of the Glidepath Project.

\subsubsection{Network Configuration}

The equivalent median number of stops recorded in both phases of testing may be due to high speeds along the corridor and relatively short distances between intersections with long signal phases. The average distance between intersections on the HCRC is $486 \mathrm{~m}$, and the median distance is $400 \mathrm{~m}$. Only three intersections on the HCRC are separated by 
more than the $500 \mathrm{~m}$ communication range, which is where fuel efficiency begins to plateau [7] [41], while three intersections on the corridor are separated by a distance below the minimum required for sufficient time to respond to speed change advice [39]. The distances between intersections may greatly affect the ability for the EcoDrive application to provide advice for the third scenario.

The distance of $350 \mathrm{~m}$ was stated by Katsaros et al. as the minimum range in which the time would be sufficient for the drivers to comply with the advice [39]. In the study that produced this distance, drivers were operating at a maximum speed of $54 \mathrm{~km} / \mathrm{h}$. It can reasonably be assumed that this distance would grow in the case of the EcoDrive testing as a speed recommendation below $50 \mathrm{~km} / \mathrm{h}$ was never observed in the testing.

The EcoDrive application is currently configured to only display the recommendation for an upcoming intersection once the previous intersection has been cleared. All three of the closely spaced intersections are in the portion of the HCRC where the speed limit is 80 $\mathrm{km} / \mathrm{h}$. The reaction time of alert drivers to simple stimulus ranges from 0.5 to 2 seconds [72]. In the case of these three intersections, should the vehicle be traveling at $60 \mathrm{~km} / \mathrm{h}$ while passing through the previous intersection, they would be arriving at the following intersection within 12 seconds, allowing for about 10 seconds of adjusted speed. The speed recommendations in the EcoDrive application were limited to $3.42 \mathrm{~m} / \mathrm{s}^{2}$, meaning that if these intersections not be able to be passed at or near the speed of the vehicle as it exited the previous intersection there is not enough space or time for the IVU to provide usable advice. For these intersections, the EcoDrive recommendations would have been significantly less effective due to the applications short communications range. 
A sensitivity analysis was performed on GLOSA functionality under various different scenarios in a study by Xai et al. which demonstrated a relationship between the communications range and fuel savings [73]. As the communication range increases as does the fuel savings, until a plateau of around $600 \mathrm{~m}$. Analysis conducted by Tielert et al. supports these findings [41]. The EcoDrive application used in this study was equipped with a single-intersection algorithm, so the device had the limitation of only perceiving the nearest upcoming intersection. The impacts of the following intersection are not considered prior to passing the immediate intersection, no matter the distance.

Coordinating GLOSA recommendations where intersections are closely spaced could reduce the number of stops and the required speed variations as the corridor is travelled.

In comparison to many of the studies presented in the literature, the cycle lengths along the HCRC are much lengthier. It was observed in Xai et al. that as cycle length increases, with the same portion of green time, the benefits of the system are reduced [38]. This study began with testing a cycle length of 50 seconds with $20 \%$ green time. The cycle lengths along the corridor during the times of testing ranged from 70 to 150 seconds.

Due to the long red phases that occur along the HCRC, the speeds required for a vehicle to pass the upcoming intersection may often be well below the acceptable speed to be traveling on the road. The increased amount of coasting that occurred is likely due to the number of recommendations that were provided based on the EcoDrive application perceiving the scenario that the driver would be required to stop at the upcoming intersection, thus providing recommendations outlined in scenarios two and four. 
The median number of stops in both phases of testing are four stops per trip. This means that on most trips drivers were required to stop at $25 \%$ of the intersections along the route regardless of the provision of additional information. This could indicate that the signals along the route are not optimized to allow green phase waves to exist along the corridor. The City of Ottawa optimizes its signal plans to accommodate the directions which see more traffic. Since the HCRC crosses three other arterials, its volume passing straight through the intersections, in comparison to those making turning movements or crossing the HCRC on a different arterial, may not be prioritized in the optimization of the whole network.

\subsubsection{Queue Length}

Another reason for the number of stops not being reduced by the use of the EcoDrive application may be due to the lack of ability to predict queue length. As stated, the algorithm provides "an additional few seconds" after the signal has changed to green to allow for dissipation of the queue which may be present at the intersection. TTS also mentions that this amount of time needs to be determined while field testing the application [54]. The ability to appropriately predict queue lengths plays a major role in the GLOSA recommendations provided in the third EcoDrive scenario. With continued field testing and more use, these predictions could be improved in a similar manner as is used to predict actuated signal phase timing based on historical data. Additionally, with an increased market penetration of connected vehicles, positional data could be used to produce real time queueing information. The improvement of the EcoDrive algorithm in relation to queue length estimation, could reduce the number of stops made by informed 
drivers, potentially resulting in an overall improvement in the effectiveness of the application. 


\section{Chapter 9 Summary and Conclusions}

This dissertation has demonstrated that through the use of a connected vehicle technology the GHG emissions of a test vehicle travelling along an arterial roadway with semiactuated traffic signals was reduced by $9.62 \%$. On a $95 \%$ confidence interval, this resulted in a range of a $1.02 \mathrm{~kg} / \mathrm{h}$ to $1.74 \mathrm{~kg} / \mathrm{h}$ reduction in $\mathrm{CO} 2$ equivalent produced with the use of the EcoDrive application. This application produced driving recommendations based off the vehicle's current position and real-time SPaT and MAP data for upcoming intersections. The two-way wireless connectivity was facilitated through a 4G LTE cellular network. The EcoDrive application provided velocity recommendations to drivers allowing for adjustments to be made to driving behaviours which resulted in fuel savings and reduced GHG emissions. The fuel savings obtained by drivers with the use of the EcoDrive application were $9.64 \%$ over the uninformed driver. Through bootstrapping, the $95 \%$ confidence interval demonstrated fuel savings between $0.38 \mathrm{~L} / \mathrm{h}$ to a $0.65 \mathrm{~L} / \mathrm{h}$ while receiving driving advice. While the use of this application provided environmental and economic benefits, no significant negative effects were observed on the time it took to travel the corridor.

The testing was conducted on a four-lane arterial roadway that connects the city of Ottawa from east-to-west, including the crossing of a bridge. Each test trip consisted of the crossing of 12 connected semi-actuated signals. These intersections were spaced between $170 \mathrm{~m}$ and $1.2 \mathrm{~km}$. Daily traffic counts were recorded periodically throughout the testing in various different locations. The averaged volumes observed from these traffic counts were between 35,600 and 57,600 vehicles per day. It is evident that in a 
real-world urban environment there is a presence of large variations in traffic volume and that wide ranges of spacing between intersections can be expected. As opposed to results presented with strict conditions imposed by simulations or testbed analysis, these results are presented as the effectiveness of a connected vehicle technology when applied to a real-world arterial under normal conditions.

All testing trips of the corridor took place in off-peak periods, during business hours, and under clear dry conditions. It is known that velocity planning applications have limited effects when heavy traffic restricts the ability for the user to follow speed recommendations. For this reason, off-peak testing was conducted and the few trips that experienced unusual traffic events causing excessive delays were removed from the analysis.

Testing was conducted in two phases for the evaluation of the use of the connected vehicle technology. In the first phase, the drivers operated the vehicle as they would normally while driving the length of the corridor without the provision of any additional information. This phase provided the baseline for comparison. The second phase of testing was conducted with the use of the EcoDrive IVU mounted on the vehicle's dash, providing velocity planning information to the driver based on their current position and the upcoming signal's real time SPaT data.

GPS data was collected at a second resolution, for all trips while the vehicle traversed the connected corridor, through an antenna attached to the roof of the test vehicle. The latitude, longitude, height above sea level, and time associated with each data point was analyzed to provide the vehicle's speed, acceleration and the grade of the road on a 
second-by-second basis. These factors were used to compute the data point's VSP, which indicates the vehicle's instantaneous engine power outputs. The second-by-second VSP information is crucial for the method of emissions modeling used.

With the use of state of the art microscopic emissions modeling software, the estimates for the amount of emissions produced and fossil fuel consumed were able to be accurately estimated for each trip. The emissions model was first tailored to replicate the Ottawa area and the vehicle used in testing. From these outputs, the average rates of emissions produced and fuel consumed were able to be calculated for each phase of testing.

Statistical analysis was performed on the three main values, fuel consumption, GHG emissions, and average trip speed. Additionally, the average fraction of time spent in various modes of operation and the average number of stops made per trip were analyzed to provide insight as to why the emissions and fuel consumption were reduced with the use of the connected vehicle technology. It was found that the average fraction of time spent per trip coasting at low and moderate speed was increased by $31.45 \%$ and $21.45 \%$ respectively, while the average fraction of time spent braking per trip was reduced by $17.33 \%$. These results can be attributed to the EcoDrive application causing the driver to reduce their speeds well in advance of having to stop at an intersection.

The messages delivered by the application allowed drivers to confidently reduce their speed or coast towards intersections. However, the application was not able to provide a reduction in the average number of stops per trip. This was likely due to the priorities present in network optimization and the lack of response time available between closely 
spaced intersections. In spite of that, benefits in terms of fuel savings and reduced emissions production were observed while the drivers were receiving information based of real-time SPaT data and their current position. These findings stand out from current literature as real-world testing was conducted over a period of time in a live traffic environment containing semi-actuated traffic signals.

Future iterations of similar velocity planning algorithms should place a high importance on the queue predicting model. Estimations provided based on historical data, in a similar manner to those used to predict green time at the semi-actuated signals, could improve the GLOSA recommendation by adjusting the position of the stop line. This adjustment could produce results similar to those seen in the literature where the number of stops observed were reduced, providing greater fuel savings and emissions reductions. The addition of longer range foresight in similar applications also has the potential to provide greater benefits. The provision of recommendations on a longer range, especially where signals are tightly spaced could result in smoother speed profiles while traveling the length of the corridor. As traffic along a corridor is not always optimized for travel on that specific route, rather optimized for the network as a whole, introducing green wave technology to these applications could be beneficial. The use of SPaT data for multiple successive intersections would allow for decisions to be made on where it would be optimal to stop, should a stop along the route be inevitable. Other simulation-based studies could expand their parameters to include a mix of traffic volumes and signal spacing to allow for simulations to more realistically represent true arterial conditions. 
In conclusion, Eco-drive-based connected vehicle-to-infrastructure applications have the ability to provide substantial benefits with little market penetration. As shown through this study, an individual vehicle operating in normal traffic conditions on an arterial road can experience fuel savings and reduced GHG emission, by $9.64 \%$ and $9.62 \%$ respectively, without compromising travel time. Rather than influencing the number of stops, the EcoDrive application's success was realized through an increased amount of time spent coasting and a reduction in braking.

Though EcoDrive here has been evaluated based on the performance of a single vehicle, the implementation of this technology on a large scale would allow for benefits to be achieved my many users. This technology is more easily implemented in the short term than many other solutions provided in the literature, in the sense of the ability to be rapidly implemented using connectivity already present and aftermarket IVU devices that are readily available. The extrapolation of these improvements to a market penetration, especially when coupled with other connected and automated applications, has the potential to reduce the amount of GHG emissions produced by personal transportation which would help bring Canada closer to achieving its climate change commitments. 


\section{Chapter 10 References}

[1] Environment and Climate Change Canada , 04 2017. [Online]. Available: http://www.ec.gc.ca/indicateurs-indicators/F60DB708-6243-4A71-896B6C7FB5CC7D01/GHGEmissions_EN.pdf. [Accessed 0501 2018].

[2] Government of Canada, "Road traffic and air pollution," 0807 2016. [Online]. Available: https://www.canada.ca/en/health-canada/services/air-quality/roadtraffic-air-pollution.html.

[3] IPCC, "Report prepared for Intergovernmental Panel on Climate Change by Working Group I," 1990. [Online]. Available:

https://www.ipcc.ch/publications_and_data/publications_ipcc_first_assessment_1 990_wg1.shtml. [Accessed 2017].

[4] Government of Canada, "Pan-Canadian Framework on Clean Growth and Climate Change," 2017. [Online]. Available: https://www.canada.ca/en/environmentclimate-change/services/climate-change/greenhouse-gas-emissions/seventhnational-communication-third-biennial-report.html.

[5] U.S. Department of Transportation, Revised Departmental Guidance on Valuation of Travel Time in Economic Analysis, Washington, 2011.

[6] K. A. Small, "Valuation of Travel Time," Economics of Transportation, vol. 1, no. 1, pp. 2-14, 2012.

[7] H. Xia, "Eco-Approach and Departure Techniques for Connected Vehicles at Signalized Traffic," UC Riverside, Ann Arbor, 2014.

[8] D. Schrank, B. Eisele and T. Lomax, "TTI's 2012 Urban Mobility Report," Texas A\&M Transportation Institute, Texas, 2012.

[9] U.S. Department of Transportation, "The intelligent transportation systems for traffic signal control deployment benefits and lessons learned," Washington, DC, , 2007. [Online]. Available:

http://www.its.dot.gov/jpodocs/repts_te/14321_files/a1019-tsc_digital. [Accessed 2017].

[10] G. De Nunzio, C. Canudas de Wit, P. Moulin and D. Di Domenico, "Eco-driving in urban traffic networks using traffic signals information," International Journal 
of Robust and Nonlinear Control, vol. 26, no. 6, pp. 1307-1324, 2015.

[11] T. Onoda, "IEA policies - G8 recommendations and an afterwards," in Energy Policy 37, pp. 3822-3831, Paris, 2009.

[12] "Ecodriving Short-duration training for licensed drivers Experiences and results from the ECOWILL project," Intelligent Energy Europe, 2013.

[13] Government of Canada, "Auto\$mart D.E.N. Drivers Educator's Newsletter," 05 2017. [Online]. Available:

http://www.nrcan.gc.ca/energy/efficiency/transportation/commercialvehicles/reports/7601.

[14] Natural Resources Canada, "Fuel-efficient Driving Techniques," 022016. [Online]. Available: http://www.nrcan.gc.ca/energy/efficiency/transportation/carslight-trucks/fuel-efficient-driving-techniques/7507. [Accessed 2017].

[15] North Carolina Department of Transportation, "Drive More Efficiently," 012018. [Online]. Available:

https://www.ncdot.gov/travel/drivegreen/drive_more_efficiently.html.

[16] J. Chong, "Automated nd Connected Vehciles: Status of the Technology and Key Policy Issues for Canadian Governments" Library of Parliament, Ottawa, 2909 2016. [Online]. Available: https://lop.parl.ca/Content/LOP/ResearchPublications/2016-98-e.pdf. [Accessed $16102017]$.

[17] M. Parent, "Automated Vehicles: Autonomous or Connected?," in 2013 IEEE 14th International Conference on Mobile Data Management, Milan, 2013.

[18] A. Wyglinski, "Connectivity: The Essential Ingredient for Reliable, Safe Autonomous Vehicles," IEEE Transmitter, 11042017.

[19] Z. Xu and et A1., "DSRC versus 4G-LTE for Connected Vehicle Applications: A Study on Field Experiments of Vehicular Communication Performance, " Journal of Advanced Transportation, vol. 27, pp. 10, 23082017.

[20] S. Mandava, K. Boriboonsomsin and M. Barth, "Arterial Velocity Planning based on Traffic Signal Information under Light Traffic Conditions," in 12th International IEEE Conference on Intelligent Transportation Systems, , St. Louis, MO, 2009. 
[21] Ford Motor Company of Canada, Limited, "SYNC® Connect powered by FordPass ${ }^{\mathrm{TM}}, "$ 2018. [Online]. Available: https://www.fordpass.ca/en_ca/syncactivation.html.

[22] About LG Electronics, Inc., "LG AND VOLKSWAGEN COMMIT TO JOINTLY DEVELOP CONNECTED CAR PLATFORM," [Online]. Available: http://www.lg.com/ae/press-release/lg-and-volkswagen-commit-to-jointlydevelop-connected-car-platform.

[23] General Motors, "GM First to Offer New AT\&T Connected Car Data Plans for Business Customers," 2018. [Online]. Available: http://www.gm.com/mol/m2017-may-0516-data-plans.html.

[24] Traffic Technology Solutions, LLC, "EcoDrive 12V Project - Connected Vehicle Proof of Concept," Beeverton, 2017.

[25] S. Gong, J. Shen and L. Du, "Constrained Optimization and Distributed Computation Based Car Following Control of a Connected and Autonomous Vehicle Platoon.," Transportation Research Part B: Methodological 94, pp. 314334, 2016.

[26] L. Y. Wang, A. Syed, G. G. Yin, A. Pandya and H. Zhang, "Control of vehicle platoons for highway safety and efficient utility: Consensus with communications and vehicle dynamics," Journal of Systems Science and Complexity, vol. 27, no. 4, p. 605-631, 2014.

[27] S. Caixing and Z. Jianhua, "Research of the multi-way connectivity probability for platoon-based vehicle-to-infrastructure communication network," The Journal of China Universities of Posts and Telecommunications, vol. 23, no. 1, pp. 1-7, 2016.

[28] G. Sharon, M. W. Levin, J. P. Hanna, T. Rambha, S. D. Boyles and P. Stone, "Network-wide adaptive tolling for connected and automated vehicles," Transportation Research Part C: Emerging Technologies, pp. 142-157, 2017.

[29] J. Joshi, A. Singh, L. G. Moitra and M. J. Deka, "DASITS: Driver Assistance System in Intelligent Transport System," in 30th International Conference on Advanced Information Networking and Applications Workshops (WAINA), CransMontana, 2016.

[30] California PATH Program Institute of Transportation Studies, "Investigating the Potential Benefits of Broadcasted Signal Phase and Timing (SPaT) Data under 
IntelliDriv," University of California, Berkeley, 2011.

[31] Y. Feng, "Intelligent Traffic Control in a Connected Vehicle Environment," University of Arizona, Tucson, 2015.

[32] Z. Liu and K. Kircher, "Comparison of a time- and a speed-based traffic light assistance system," Cognition, Technology \& Work, pp. 93-103, 2017.

[33] National Research Council Transportation Research Board, "Field Evaluation of Vehicle-to-Infrastructure Communication-Based Eco-Driving Guidance and EcoSignal System," in Humaist Conference, The Hague, 2018.

[34] K. Dresner and P. Stone, "A Multiagent Approach to Autonomous Intersection Management," Journal of Artificial Intelligence Research, pp. 591-656, 2008.

[35] Q. Jin, "Eco-Friendly Agent Based Advanced Traffic Management Techniques in a Connected Vehicle Environment," UC Riverside, Ann Arbor, 2015.

[36] G. A. Ubiergo and W.-L. Jin, "Mobility and environment improvement of signalized networks through Vehicle-to-Infrastructure (V2I) communications," Transportation Research Part C: Emerging Technologies, pp. 70-82, 2016.

[37] H. Xia, K. Boriboonsomsin and M. Barth, "Dynamic Eco-Driving for Signalized Arterial Corridors and Its Indirect Network-Wide Energy/ Emissions Benefits," Journal of Intelligent Transportation Systems, vol. 17, no. 1, pp. 31-41, 2013.

[38] H. Xai, G. Wu, K. Boriboonsomsin and M. Barth, "Development and Evaluation of an Enhansed Eco-Approach Traffic Signal Application for Connected Vehicles," in 16th International IEEE Annual Conference on Interlligent Transportation Systems, The Hague, 2013.

[39] K. Katsaros, R. Kernchen, M. Dianati and D. Rieck, "Performance study of a Green Light Optimized Speed Advisory (GLOSA) Application Using an Integrated Cooperative ITS Simulation Platform," Istanbul, 2011.

[40] A. Stevanovic, J. Stevanovic and C. Kergaye, "Comparative Evaluation of Benefits from Traffic Signal Retiming and Green Light Optimized Speed Advisory Systems," Transportation Research Board 93rd Annual Meeting, Washington, 2014.

[41] T. Tielert, M. Killat, H. Hartenstein, R. Luz, S. Hausberger and T. Benz, "The impact of traffic-light-to-vehicle communication on fuel consumption and 
emissions," in Internet of Things (IOT), Tokyo, 2010.

[42] B. Asadi and A. Vahidi, "Predictive Cruise Control: Utilizing Upcoming Traffic Signal Information for Improving Fuel Economy and Reducing Trip Time," IEEE Transactions On Control Systems Technology, pp. 707-714, 2011.

[43] M.-A. Lebre et al., "Real scenario and simulations on GLOSA traffic light system," 22nd ITS World Congress, Bordeaux, 2015.

[44] O. Altan, G. Wu, M. Barth, K. Boriboonsomsin and J. Stark, "GlidePath: EcoFriendly Automated Approach and Departure at Signalized Intersections," IEEE Transactions On Intelligent Vehicles, vol. 2, no. 4, pp. 266-277, 122017.

[45] H. Chen, H. A. Rakha, A. Loulizi, I. El-Shawarby and M. H. Almannaa, "Development and Preliminary Field Testing of an In-Vehicle Eco-Speed Control System in the Vicinity of Signalized Intersections," in International Federation of Automatic Control, vol. 46, no. 3, pp. 249-254, 2016.

[46] Bureau de l'éfficacité et de l'innovation énergétique, "Eco-Driving Training Pilot," Ministère des Ressources Naturelles et de la Faune, Québec, 2011.

[47] Yiping Wu; Xiaohua Zhao; Jian Rong;, "The Long-Term Effectiveness Of EcoDriving Traning: A Pilot Study," in Proceedings of the Eighth International Driving Symposium on Human Factors in Driver Assessment, Training and Vehicle Design, Beijing, 2015.

[48] J. Lee, S. Gutesa, B. Dimitrijevic, Y. Zhang, L. Spasovic and J. Singh, "Deployment and Field Evaluation of In-Vehicle Traffic Signal Advisory System (ITSAS)," Information, vol. 8, no. 72, pp. 1-13, 2017.

[49] C. Spiegelman, L. Rilett and E. Park, Transportation Statistics and Microsimulation. Chapman \& Hall, New York, 2011.

[50] R. Roess, E. Prassas and W. McShane, Traffic Engineering. Fourth Edition, Prentice Hall., 2011.

[51] M. Anderson, "Traffic Signal Timing," City of Ottawa, Transportation Services Department, Traffic Operations Unit, 2017.

[52] City of Ottawa, "Transportation System Management," 2502 2017. [Online]. Available: https://ottawa.ca/en/residents/transportation-andparking/traffic/transportation-system-management. 
[53] City of Ottawa, "City infrastructure connects vehicles to traffic signals for a safer, smoother, greener trip," 1611 2017. [Online]. Available:

https://ottawa.ca/en/news/city-infrastructure-connects-vehicles-traffic-signalssafer-smoother-greener-trip.

[54] Traffic Technology Sollutions, "EcoDrive App Front-End Design," Beaverton, $2017 b$.

[55] Leica Geosystems AG, "TechnicalReference Manual," www.leicageosystems.com, Heerbrugg, 1999.

[56] esri, "How To: Calculate Decimal Degrees from Degrees Minutes Seconds (DMS) manually," 55 2016. [Online]. Available: https://support.esri.com/en/technicalarticle/000007058.

[57] Natural Resources Canada, "The UTM Grid - Universal Transverse Mercator Projection," 2508 2016. [Online]. Available: http://www.nrcan.gc.ca/earthsciences/geography/topographic-information/maps/9779.

[58] City of Ottawa, "Roads," 3009 2017. [Online]. Available: http://data.ottawa.ca/dataset/roads.

[59] Z. Yao, H. Wei, H. Perugu, H. Liu and Z. Li, "Sensitivity analysis of project level MOVES running emission rates for light and heavy duty vehicles," Journal of Traffic and Transportation Engineering, vol. 1, no. 2, pp. 81-96, 2014.

[60] Office of the Assistant Secretary for Research and Technology, "GlidePath Prototype ApplicationAutomated Eco-Friendly Cruise Control using Wireless V2I Communications at Signalized Intersections," 2810 2015. [Online]. Available: https://www.its.dot.gov/research_archives/aeris/pdf/GlidePathWebinar.pdf.

[61] Office of Transportation and Air Quality, "EPA Releases MOVES2014a Mobile Source Emissions Model: Questions and Answers," November 2015. [Online]. Available: https://nepis.epa.gov/Exe/ZyPDF.cgi?Dockey=P100NNR0.txt.

[62] US EPAb, "MOVES2014 and MOVES2014a Technical Guidance: Using MOVES to Prepare Emission Inventories for State Implementation Plans and Transportation Conformity," 10 2015. [Online]. Available: https://nepis.epa.gov/Exe/ZyPDF.cgi?Dockey=P100NN9L.txt.

[63] United States Environmental Protection Agency, "Module 8Project-Scale Analyses," 08 2017. [Online]. Available: https:/www.epa.gov/moves/moves- 
training-sessions.

[64] Z. Yao, W. Heng, H. Liu and Z. Li, "Statistical Vehicle Specific Power Profiling for Urban Freeways," Social and Behavioral Sciences, vol. 96, p. 2927 - 2938, 6 102013.

[65] DMVAdvisors, "New York Emissions Testing," 2017. [Online]. Available: https://www.dmv.com/ny/new-york/emissions-testing.

[66] Government of Ontario, "Drive Clean test," 0812 2017. [Online]. Available: https://www.ontario.ca/page/drive-clean-test.

[67] United States Environmental Protection Agency," Light-Duty Exhaust Emission Rates in MOVES2010," 06 2016. [Online]. Available: https://www.epa.gov/sites/production/files/2016-06/documents/light-dutyexhaust-moves-2011.pdf.

[68] Exxon Mobil Corporation, "Ethanol," 09 2017. [Online]. Available: https://www.esso.ca/en/ethanol.

[69] Governement of Canada, "Hourly Data Report for September 13, 2017," 092017. [Online]. Available:

http://climate.weather.gc.ca/climate_data/hourly_data_e.html?StationID $=49568 \& t$ imeframe $=1 \&$ StartYear $=1840 \&$ EndY $\overline{\text { ear}}=2017 \&$ type=line $\&$ MeasTypeID=relhum $\&$ Year $=2017 \&$ Month $=9 \&$ Day $=13 \#$.

[70] Statistics Canada, "57-601-X Energy Statistics Handbook, Appendix A: Conversion factors," 2008. [Online]. Available: http://www.statcan.gc.ca/pub/57601-x/2012001/appendix-appendice1-eng.htm.

[71] R Core Team, $R$ : A language and environment for statistical computing, Vienna, 2017.

[72] Transportation Association of Canadda, "1.2.2 Human factors," in Geometric Design Guide for Canadian Roads, 2009.

[73] H. Xia, G. Wu, K. Boriboonsomsin and M. J. Barth, "Development and Evaluation of an Enhanced Eco-Approach Traffic Signal Application for Connected Vehicles," in International IEEE Annual Conference on Intelligent Transportation Systems, The Hague, 2013.

[74] OC Transpo, "System Map," 1701 2016. [Online]. Available: 
http://www.octranspo.com/images/files/maps/system_map/systemmap.pdf.

[75] geoOttawa, "GeoOttawa," 2017. [Online]. Available:

http://maps.ottawa.ca/geoottawa/.

[76] City of Ottawa,g, "Urban Trucking Routes," 2017. [Online]. Available:

http://documents.ottawa.ca/sites/documents.ottawa.ca/files/urban_Truck_Routes_ 2017_feb_en.pdf.

[77] City of Ottawa, g2, "Rural truck Routes," 2017. [Online]. Available:

http://documents.ottawa.ca/sites/documents.ottawa.ca/files/rural_Truck_Routes_2 017_feb_en.pdf.

[78] A. Khan, "Eco-Drive Technology Evaluation for Commercial Vehicles in the City of Ottawa," Ottawa, 2018. 


\section{Appendix A Data Formatting Equations}

The GPS point feature files were initially evaluated in Microsoft Excel.

Using the $\mathrm{X}$ and $\mathrm{Y}$ coordinates output by ArcMap; the horizontal distance from the previous point was calculated using Equation 3.

Equation 3: Horizontal distance: Dxy $=\sqrt{\left(\mathrm{x}_{\mathrm{n}-1}-\mathrm{x}_{\mathrm{n}}\right)^{2}+\left(\mathrm{y}_{\mathrm{n}-1}-\mathrm{y}_{\mathrm{n}}\right)^{2}}$.

The vertical distance from the previous point was calculated using Equation 4.

Equation 4: Vertical distance: $\mathrm{Dz}=\mathrm{z}_{\mathrm{n}}-\mathrm{z}_{\mathrm{n}-1}$

The $3 \mathrm{D}$ distance traveled from the previous point was calculated using Equation 5.

Equation 5 3D Distance: $\operatorname{Dxyz}=\sqrt{\left(x_{n-1}-x_{n}\right)^{2}+\left(y_{n-1}-y_{n}\right)^{2}+\left(z_{n-1}-z_{n}\right)^{2}}$

For each separate trip the $3 \mathrm{D}$ distances were combined to calculate the total trip length using Equation 6.

Equation 6: Total Trip Length: $\mathrm{D}_{\text {total }}=\sum \mathrm{D}_{\mathrm{xyz}}$

The individual trip length were converted to miles for the MOVES inputs using Equation 7.

Equation 7: Trip Length in Miles: $\mathrm{D}_{\text {total }} * \frac{100 \mathrm{~cm}}{1 \mathrm{~m}} * \frac{1 \mathrm{in}}{2.54 \mathrm{~cm}} * \frac{1 \mathrm{ft}}{12 \mathrm{in}} * \frac{1 \mathrm{mile}}{5280 \mathrm{ft}}$

The instantaneous speeds were calculated for each GPS point using Equation 8.

Equation 8: Speed: $v_{m / s}=\frac{\operatorname{Dxyz}_{n}-D x y z_{n-1}}{t_{n}-t_{n-1}}$

The acceleration at each point was calculated using Equation 9.

Equation 9: Acceleration: $a=\frac{v_{n}-v_{n-1}}{t_{n}-t_{n-1}}$

The slope at each point was calculated using Equation 10.

Equation 10: Slope: $S=\frac{D z}{D x y}$

For the MOVES inputs, the average slope of the trip is required. This was calculated using Equation 11.

Equation 11: Average Slope: $S_{\text {ave }}=\sum \operatorname{slope}_{\mathrm{i}} *\left(\frac{\text { Dxyz }_{\mathrm{i}}}{\text { Dtotal }}\right)$

The speed in Meters per second was converted to Kilometers per hour using Equation 12.

Equation 12: Speed in $\frac{\mathrm{Km}}{\mathrm{hr}}: \mathrm{V}_{\frac{\mathrm{km}}{\mathrm{h}}}=\mathrm{V}_{\frac{\mathrm{m}}{\mathrm{s}}} * \frac{\mathrm{km}}{1000 \mathrm{~m}} * \frac{3600 \mathrm{~s}}{1 \mathrm{~h}}$

The speed in miles per hour, as required by the MOVES inputs, was calculated using Equation 13. 
Equation 13: Speed in Miles per Hour: $\mathrm{V}_{\mathrm{MPH}}=\mathrm{V}_{\frac{\mathrm{s}}{\mathrm{s}}} * \frac{100 \mathrm{~cm}}{1 \mathrm{~m}} * \frac{1 \mathrm{in}}{2.54 \mathrm{~cm}} * \frac{1 \mathrm{ft}}{12 \mathrm{in}} * \frac{1 \mathrm{mile}}{5280 \mathrm{ft}} *$ $\frac{60 \mathrm{~s}}{1 \mathrm{~min}} * \frac{60 \mathrm{~min}}{1 \mathrm{hr}}$

The temperatures in Celsius from the Government of Canada Archives were converted to Fahrenheit using Equation 14

Equation 14: $\mathrm{F}^{\mathrm{o}}=\mathrm{C}^{\mathrm{o}} * \frac{9}{5}+32$

The Moves outputs were converted to generic metrics with the use of the following two equations

Equation 15: $\mathrm{CO}_{2}$ equivalent conversion $\frac{\operatorname{CO} 2 \mathrm{E}(\mathrm{g})}{\mathrm{Run}} * \frac{\mathrm{Kg}}{1000 \mathrm{~g}} * \frac{\mathrm{Run}}{\text { trip time }(\mathrm{h})}=\mathrm{CO}_{2} \mathrm{E}(\mathrm{kg} / \mathrm{h})$

Equation 16: Fossil fuel conversion: Fossil Fuel Energy consumption $(\mathrm{L} / \mathrm{h})=$

$\frac{\text { Fossil Fuel energy consumption }(\mathrm{J})}{\text { Run }} * \frac{\mathrm{MJ}}{1000000 \mathrm{~J}} * \frac{\mathrm{Run}}{\text { travel time }(\mathrm{h})} * \frac{\mathrm{L}}{35 \mathrm{MJ}}$ 


\section{Appendix B Trip Results}

Samples of Excel files for MOVES inputs

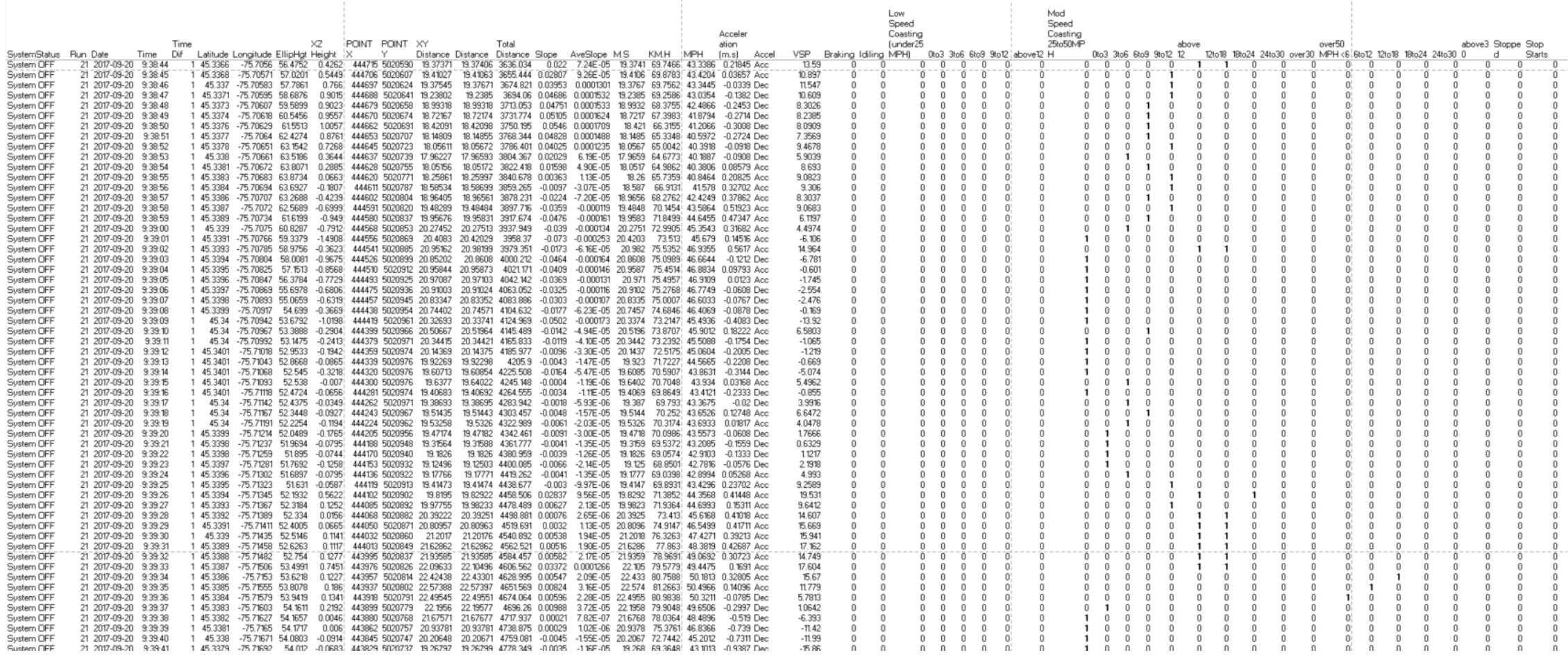

Figure 17: Sample second-by-second analysis a 
Time $X 2$ iPOINT POINT $X Y$

Total

Acceler

ation.

SystemStatus Pun Date Time Dif Latitude Longitude EllipHot Height iX Y Distance Distance Distance Slope AveSlope MS KMH MPH (m.s) Acced

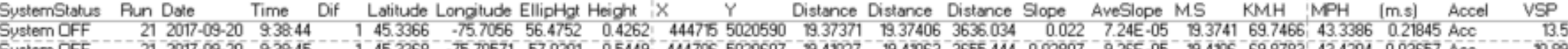

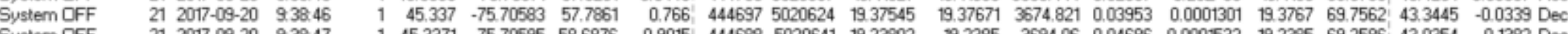
$\begin{array}{llll}\text { System OFF } & 21 & 2017-09-20 & 93647\end{array}$ System OFF $212017-09-20 \quad 93649$ System OFF $212017-09-20 \quad 938.49$ System LFF 21 2017-09-20 938.50 System OFF $\quad 21$ 2017-09-20 $9.38: 51$ System OFF 21 2017-09-20 93652 Sustem OFF $212017.09-20 \quad 936.53$ System QFF 21 2017-09.20 9.36 .54 System LFF $2112017-09-20 \quad 9.36 .55$ System LFF 21 2017-09-20 9.36 .56 System OFF $212017-09-2023057$ System OFF 21 2017-09-20 9336.58 System LFF $212017.09 .20 \quad 936.59$ System LFF 21 2017-09-20 9339.00 System DFF $\quad 21$ 2017-09-20 9.39:01 System DFF 21 2017-09-20 939.02 $\begin{array}{llll} & \text { System OFF } 21 & 2017-09-20 & 939.03\end{array}$ System DFF 21 2017-09-20 9.39 .04 $\begin{array}{llll} & \text { System DFF } 21 & 2017-09-20 & 939.05\end{array}$ $\begin{array}{llll} & \text { System DFF } 2112017-09-20 & 9.39 .06\end{array}$ $\begin{array}{llll}\text { System DFF } 21 & 2017-09-20 & 939.07\end{array}$ System OFF 21 2017-09-20 939908 System OFF 21 2017-09-20 9939.09

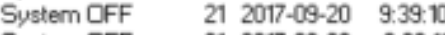

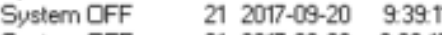
System DFF $\quad 21$ 2017-09-20 9:39:12 System OFF $\quad 21$ 2017-09-20 9.39:13 System OFF 21 2017-09-20 $9: 39: 14$ System DFF 21 2017-09-20 $\quad 9: 39: 15$ $212017-09-20 \quad 9: 39: 10$ Sustem DFF 21 2017-09-20 $\quad 9: 39: 17$

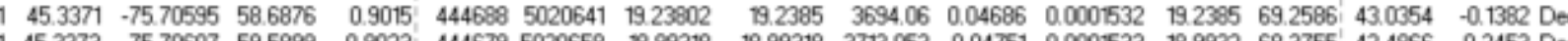

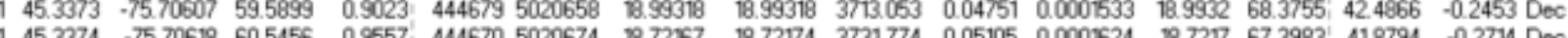

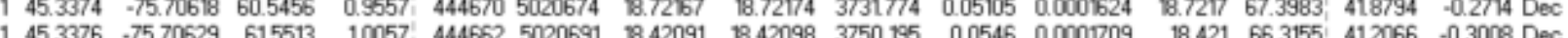

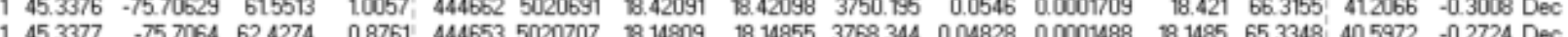

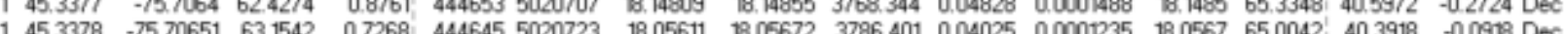

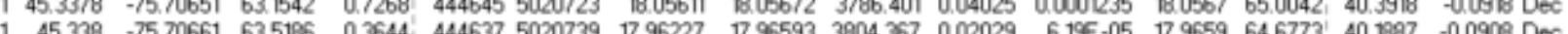

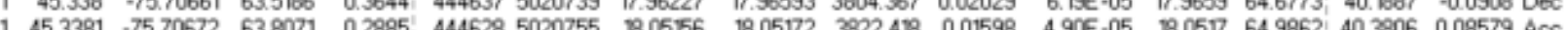

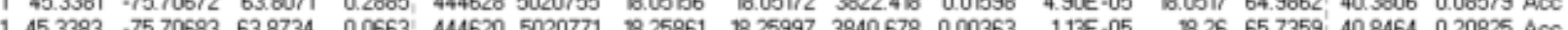

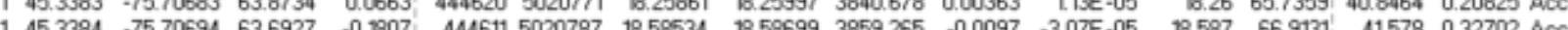

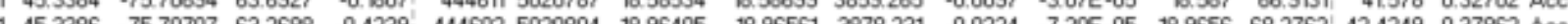

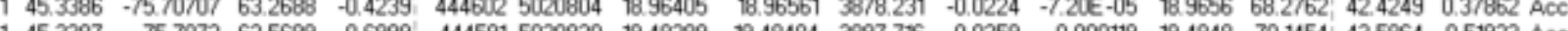

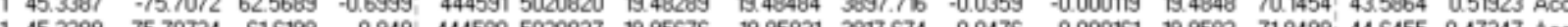

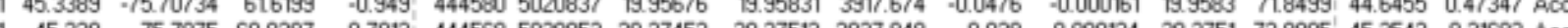
\begin{tabular}{llllllllllllllll}
\hline & 45.339 & -75.7075 & 60.8287 & -0.7912 & 444568 & 5020853 & 20.27452 & 20.27513 & 3937.949 & -0.039 & -0.000134 & 20.2751 & 72.9905 & 45.3543 & 0.31682 \\
\hline
\end{tabular}

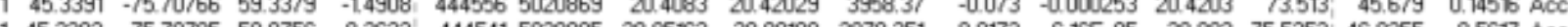



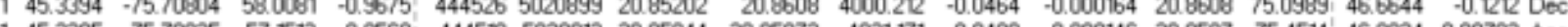

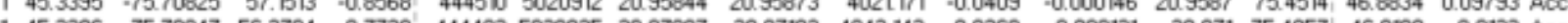



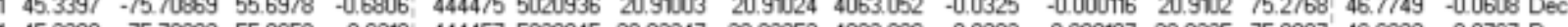

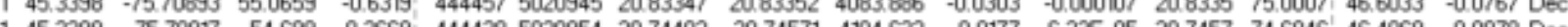

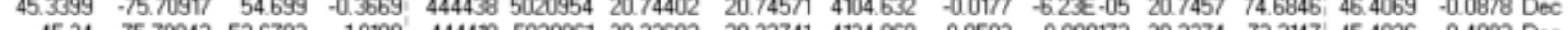
$\begin{array}{lllllllllllllllll}45.34 & -75.70942 & 53.6792 & -10198 & 444419 & 5020961 & 2032693 & 20.33741 & 4124.969 & -0.0502 & -0.000173 & 20.3374 & 73.2147 & 45.4936 & -0.4083 & \text { Dec }\end{array}$

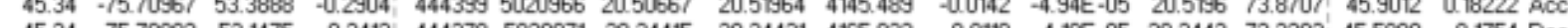

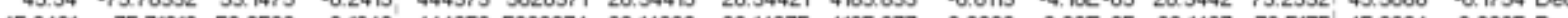
$\begin{array}{llllllllll} & \end{array}$


$\begin{array}{llll} & \end{array}$ $\begin{array}{llllll} & \end{array}$

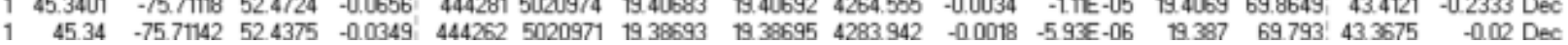
10.897 11547 10.609
8.3026 83026 8.2385 7,3569 94678 59039 8.693 9.0823 9.306 83037 90683 6. 1197 4.4974 $-6.106$ 14.964 $-6.781$ $-0.601$ $-1745$ $-2.554$ $-2.476$ $-0.169$ -13.92
6.5803 $-1065$ $-1.219$ $-0.669$ -5.074
5.4962 5.4962
-0.855 3.9916

Figure 18: Sample second-by-second analysis b 


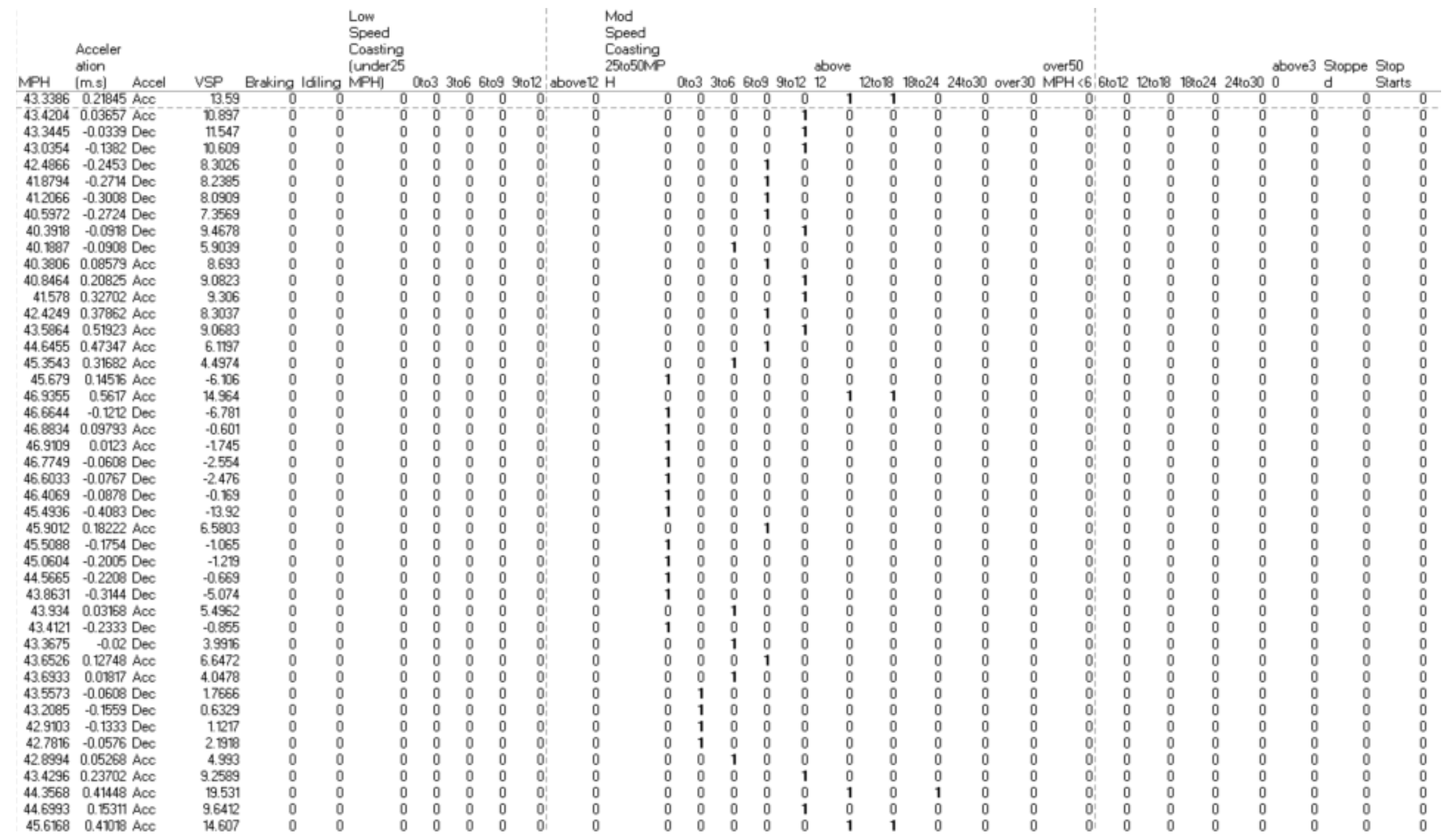

Figure 19: Sample second-by-second analysis c 


\section{Initial visualizations of the trip data}



Figure 20: Initial trip result visualization

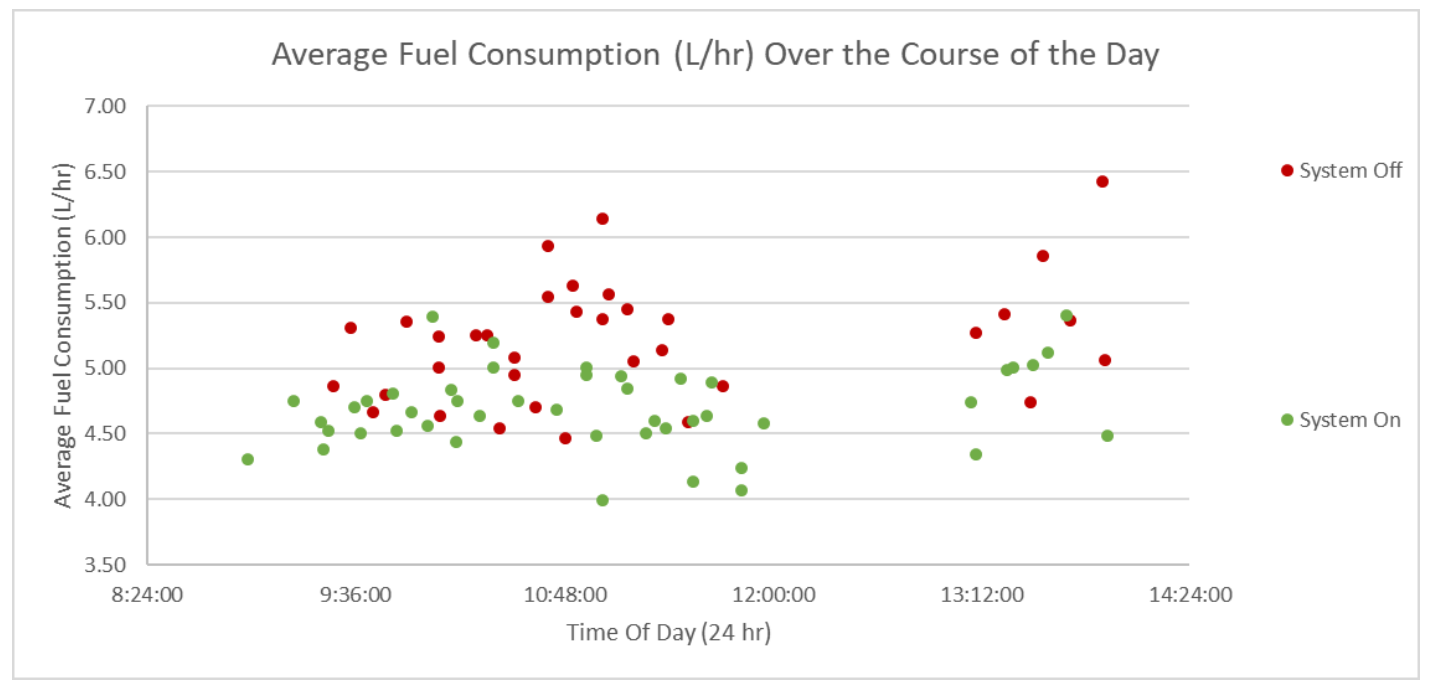

Figure 21: Initial trip visualization at times of day 


\section{Summary results with Average speeds and MOVES outputs}

\begin{tabular}{|c|c|c|c|c|c|c|c|c|c|c|c|c|}
\hline Prefix & $\begin{array}{l}\text { Run } \\
\text { ID }\end{array}$ & Date & Start Time & End Time & $\begin{array}{l}\text { Trip } \\
\text { Duration }\end{array}$ & $\begin{array}{l}\text { Trip } \\
\text { Distance } \\
\text { (m) }\end{array}$ & $\begin{array}{l}\text { Average } \\
\text { Trip } \\
\text { Speed } \\
(\mathrm{km} / \mathrm{h})\end{array}$ & Reason for being excluded & $\begin{array}{l}\mathrm{CO} 2 \\
\text { equivalent } \\
\text { produced } \\
\text { per run }(\mathrm{g})\end{array}$ & $\begin{array}{l}\mathrm{CO} 2 \\
\text { Equivalent } \\
\text { Produced } \\
\text { (Kg/hr) }\end{array}$ & $\begin{array}{l}\text { Average } \\
\text { Fossil Fuel } \\
\text { energy } \\
\text { consumption } \\
\text { per run (J) }\end{array}$ & $\begin{array}{l}\text { Average } \\
\text { Fossil Fuel } \\
\text { energy } \\
\text { consumption } \\
\text { (L/hr) }\end{array}$ \\
\hline OFF & 1 & 13-Sep & $10: 04: 33$ & $10: 12: 24$ & 0:07:51 & 5893.07 & 45.04 & & 1628.72 & 12.45 & 21258900 & 4.64 \\
\hline OFF & 2 & 13-Sep & 10:16:59 & 10:24:04 & 0:07:05 & 5884.04 & 49.84 & & 1665.63 & 14.11 & 21745600 & 5.26 \\
\hline OFF & 3 & 13-Sep & 10:25:11 & 10:34:27 & 0:09:16 & 5899.28 & 38.2 & & 1883.04 & 12.19 & 24577600 & 4.55 \\
\hline OFF & 4 & 13-Sep & 10:37:53 & $10: 46: 42$ & $0: 08: 49$ & 5904.77 & 40.18 & & 1857.3 & 12.64 & 24243300 & 4.71 \\
\hline OFF & 5 & 13-Sep & 10:47:51 & 10:56:33 & $0: 08: 42$ & 5908.29 & 40.75 & & 1738.9 & 11.99 & 22695600 & 4.47 \\
\hline OFF & 6 & 13-Sep & 11:01:01 & 11:08:11 & 0:07:10 & 5882.34 & 49.25 & & 1724.96 & 14.44 & 22521100 & 5.39 \\
\hline OFF & 7 & 13-Sep & 11:09:37 & $11: 18: 25$ & 0:08:48 & 5898.12 & 40.21 & & 2147.76 & 14.64 & 28041600 & 5.46 \\
\hline OFF & 8 & 13-Sep & $11: 21: 33$ & $11: 29: 25$ & $0: 07: 52$ & 5895.24 & 44.96 & & 1810.66 & 13.81 & 23638200 & 5.15 \\
\hline OFF & 9 & 13-Sep & $11: 30: 30$ & $11: 39: 20$ & $0: 08: 50$ & 5894.58 & 40.04 & & 1814.87 & 12.33 & 23688300 & 4.6 \\
\hline OFF & 10 & 13-Sep & $11: 42: 26$ & 11:53:17 & $0: 10: 51$ & 5900.75 & 35.35 & & 2181.09 & 13.06 & 28471500 & 4.87 \\
\hline OFF & 11 & 13-Sep & $13: 28: 29$ & 13:37:51 & $0: 09: 22$ & 5891.98 & 37.74 & & 1988.36 & 12.74 & 25954200 & 4.75 \\
\hline OFF & 12 & 13-Sep & 13:41:14 & 13:52:07 & $0: 10: 53$ & 5900.42 & 32.53 & Two light cycles to get through at Prince of Whales & 2269.68 & 12.51 & 29625500 & 4.67 \\
\hline OFF & 13 & 13-Sep & 13:53:26 & 2:03:26 & 0:10:01 & 5891.76 & 35.29 & & 2878.93 & 17.24 & 37596500 & 6.43 \\
\hline OFF & 14 & 13-Sep & 2:06:29 & 2:14:45 & 0:08:16 & 5893.1 & 42.77 & & 1797.94 & 13.05 & 23469800 & 4.87 \\
\hline OFF & 15 & 13-Sep & $2: 15: 43$ & 2:23:44 & 0:08:01 & 5896.28 & 44.13 & & 1820.66 & 13.63 & 23768200 & 5.08 \\
\hline OFF & 16 & 13-Sep & 2:27:31 & 2:36:12 & $0: 08: 41$ & 5898.65 & 40.76 & & 1985.73 & 13.72 & 25923400 & 5.12 \\
\hline OFF & 17 & 13-Sep & 2:37:47 & 2:48:10 & $0: 10: 23$ & 5879.51 & 33.97 & & 2094.46 & 12.1 & 27336600 & 4.51 \\
\hline OFF & 18 & 13-Sep & 2:52:01 & 3:00:02 & 0:08:01 & 5900.75 & 44.16 & & 1887.15 & 14.12 & 24637500 & 5.27 \\
\hline OFF & 19 & 13-Sep & 3:01:26 & $3: 10: 22$ & 0:08:56 & 5886.19 & 39.53 & & 2001.12 & 13.44 & 26123300 & 5.01 \\
\hline OFF & 20 & 13-Sep & $3: 17: 31$ & $3: 26: 58$ & $0: 09: 27$ & 5893.72 & 37.42 & & 2068.83 & 13.14 & 27005900 & 4.9 \\
\hline OFF & 21 & 20-Sep & 9:33:47 & 9:41:20 & 0:07:33 & 5886.15 & 46.67 & & 1798.91 & 14.26 & 23486000 & 5.32 \\
\hline OFF & 22 & 20-Sep & 9:45:47 & 9:56:01 & $0: 10: 14$ & 5909.78 & 34.59 & & 2201.5 & 12.89 & 28737000 & 4.81 \\
\hline OFF & 23 & 20-Sep & 10:04:12 & 10:10:58 & 0:06:46 & 5898.29 & 52.17 & & 1592.41 & 14.09 & 20789600 & 5.25 \\
\hline OFF & 24 & 20-Sep & 10:21:04 & 10:29:12 & 0:08:08 & 5908.55 & 43.5 & & 1916.42 & 14.11 & 25019400 & 5.26 \\
\hline OFF & 25 & 20-Sep & 10:30:29 & $10: 38: 54$ & $0: 08: 25$ & 5891.98 & 41.92 & & 1870.35 & 13.31 & 24415700 & 4.96 \\
\hline OFF & 26 & 20-Sep & 10:42:05 & 10:50:23 & $0: 08: 18$ & 5919.81 & 42.71 & & 2062.85 & 14.88 & 26933600 & 5.55 \\
\hline OFF & 27 & 20-Sep & 10:51:47 & 11:00:04 & $0: 08: 17$ & 5911.79 & 42.74 & & 2019.76 & 14.6 & 26369700 & 5.45 \\
\hline OFF & 28 & 20-Sep & 11:02:53 & 11:10:35 & $0: 07: 42$ & 5908.18 & 45.94 & & 1922.84 & 14.95 & 25105600 & 5.58 \\
\hline OFF & 29 & 20-Sep & 11:11:42 & $11: 20: 25$ & 0:08:43 & 5902.47 & 40.55 & & 1975.68 & 13.57 & 25791500 & 5.06 \\
\hline OFF & 30 & 20-Sep & 11:23:44 & 11:32:17 & 0:08:33 & 5890.78 & 41.26 & & 2062.51 & 14.45 & 26927700 & 5.39 \\
\hline OFF & 31 & 20-Sep & 12:36:06 & $12: 44: 48$ & $0: 08: 42$ & 5881.69 & 40.49 & Lunch hour & 2041.3 & 14.05 & 26649800 & 5.24 \\
\hline OFF & 32 & 20-Sep & 12:48:59 & $12: 57: 35$ & 0:08:36 & 5915.18 & 41.19 & Lunch hour & 2102.82 & 14.64 & 27454700 & 5.46 \\
\hline OFF & 33 & 20-Sep & $12: 58: 25$ & 13:06:43 & 0:08:18 & 5879.25 & 42.42 & Lunch hour & 1999.94 & 14.43 & 26111000 & 5.38 \\
\hline OFF & 34 & 20-Sep & 13:09:56 & 13:18:45 & 0:08:49 & 5900.68 & 40.08 & & 2084.16 & 14.16 & 27209600 & 5.28 \\
\hline OFF & 35 & 20-Sep & 13:19:42 & 13:28:44 & 0:09:02 & 5900.36 & 39.12 & & 2193.1 & 14.54 & 28633200 & 5.42 \\
\hline OFF & 36 & 20-Sep & 13:32:46 & 13:40:25 & 0:07:39 & 5896.09 & 46.14 & & 2010.42 & 15.73 & 26251300 & 5.87 \\
\hline OFF & 37 & 20-Sep & $13: 42: 27$ & 13:51:22 & 0:08:55 & 5889.86 & 39.56 & & 2146.22 & 14.41 & 28021000 & 5.38 \\
\hline OFF & 38 & 20-Sep & 13:54:22 & 2:03:49 & $0: 09: 28$ & 5900.18 & 37.4 & & 2146.22 & 13.6 & 28021000 & 5.07 \\
\hline OFF & 39 & 20-Sep & 2:05:08 & 2:12:37 & 0:07:29 & 5886.46 & 47.09 & & 1948.37 & 15.59 & 25440800 & 5.82 \\
\hline OFF & 40 & 20-Sep & 2:16:41 & 2:26:31 & 0:09:50 & 5921.77 & 36.07 & & 2361.4 & 14.38 & 30829900 & 5.37 \\
\hline OFF & 41 & 20-Sep & 3:09:05 & 3:18:05 & 0:09:00 & 5907.49 & 39.31 & & 2168.19 & 14.43 & 28303500 & 5.38 \\
\hline OFF & 42 & 20-Sep & $3: 23: 52$ & 3:34:00 & $0: 10: 08$ & 5942.79 & 35.13 & & 2360.77 & 13.96 & 30819800 & 5.21 \\
\hline OFF & 43 & 27-Sep & $9: 27: 51$ & $9: 37: 33$ & 0:09:42 & 5892 & 36.45 & & 2111.97 & 13.06 & 27568700 & 4.87 \\
\hline OFF & 44 & 27-Sep & 9:41:30 & $9: 51: 56$ & $0: 10: 26$ & 5881.85 & 33.83 & & 2178.54 & 12.53 & 28435800 & 4.67 \\
\hline OFF & 45 & 27-Sep & 9:53:10 & 10:01:28 & 0:08:18 & 5892.74 & 42.6 & & 1990.45 & 14.39 & 25986800 & 5.37 \\
\hline OFF & 46 & 27-Sep & $10: 04: 28$ & 10:14:43 & $0: 10: 15$ & 5907.61 & 34.58 & & 2297.14 & 13.45 & 29987100 & 5.02 \\
\hline OFF & 47 & 27-Sep & $10: 30: 34$ & $10: 38: 46$ & 0:08:12 & 5902.44 & 43.19 & & 1864.27 & 13.64 & 24337300 & 5.09 \\
\hline OFF & 48 & 27-Sep & 10:41:53 & 10:49:13 & $0: 07: 20$ & 5890.92 & 48.2 & & 1946.31 & 15.92 & 25414500 & 5.94 \\
\hline OFF & 49 & 27-Sep & $10: 50: 28$ & 10:58:21 & 0:07:53 & 5901.14 & 44.91 & & 1986.19 & 15.12 & 25933200 & 5.64 \\
\hline OFF & 50 & 27-Sep & 11:00:53 & 11:07:51 & 0:06:58 & 5895.72 & 50.78 & & 1915.12 & 16.49 & 25008600 & 6.15 \\
\hline DAY 4 & 0.01 & 11-Oct & 10:55:08 & 11:03:49 & 0:08:41 & 5917.8 & 40.9 & IVU not displaying suggested speed, only cycle leng & 1945.23 & 13.41 & 25393600 & 5.01 \\
\hline DAY 4 & 0.02 & 11-Oct & 11:07:05 & 11:14:56 & 0:07:51 & 5884.7 & 45 & IVU not displaying suggested speed, only cycle leng & 1737.65 & 13.26 & 22683400 & 4.95 \\
\hline DAY 4 & 0.03 & 11-Oct & 11:15:57 & 11:24:06 & 0:08:09 & 5899.3 & 43.4 & IVU not displaying suggested speed, only cycle leng & 1642.64 & 12.07 & 21439600 & 4.51 \\
\hline DAY 4 & 0.04 & 11-Oct & 11:27:48 & 11:37:08 & 0:09:20 & 5903.2 & 37.9 & IVU not displaying suggested speed, only cycle leng & 2056.95 & 13.2 & 26851300 & 4.93 \\
\hline DAY 4 & 0.05 & 11-Oct & 11:38:31 & 11:46:05 & $0: 07: 34$ & 5898.8 & 46.8 & IVU not displaying suggested speed, only cycle leng & 1658.99 & 13.13 & 21656300 & 4.91 \\
\hline
\end{tabular}

Figure 22: Summary results with Average speeds and MOVES outputs a 


\begin{tabular}{|c|c|c|c|c|c|c|c|c|c|c|c|c|}
\hline Prefix & $\begin{array}{l}\text { Run } \\
\text { ID }\end{array}$ & Date & Start Time & End Time & $\begin{array}{l}\text { Trip } \\
\text { Duration }\end{array}$ & $\begin{array}{l}\text { Trip } \\
\text { Distance } \\
\text { (m) }\end{array}$ & $\begin{array}{l}\text { Average } \\
\text { Trip } \\
\text { Speed } \\
(\mathrm{km} / \mathrm{h})\end{array}$ & Reason for being excluded & $\begin{array}{l}\mathrm{CO} 2 \\
\text { equivalent } \\
\text { produced } \\
\text { per run }(\mathrm{g})\end{array}$ & $\begin{array}{l}\mathrm{CO} 2 \\
\text { Equivalent } \\
\text { Produced } \\
\text { (Kg/hr) }\end{array}$ & $\begin{array}{l}\text { Average } \\
\text { Fossil Fuel } \\
\text { energy } \\
\text { consumption } \\
\text { per run (J) }\end{array}$ & $\begin{array}{l}\text { Average } \\
\text { Fossil Fuel } \\
\text { energy } \\
\text { consumption } \\
\text { (L/hr) }\end{array}$ \\
\hline DAY 4 & 0.06 & 5 11-Oct & $11: 48: 46$ & 11:59:52 & 0:11:06 & 5907.3 & 31.9 & 9 IVU not displaying suggested speed, only cycle leng & 2109.67 & 11.38 & 27531800 & 4.25 \\
\hline DAY 4 & 0.07 & 7 11-Oct & 12:43:04 & $12: 52: 00$ & 0:08:56 & 5898 & 39.6 & 6 Lunch Hour, IVU not displaying suggested speed, on & 1961.24 & 13.15 & 25601700 & 4.91 \\
\hline DAY 4 & 0.08 & 3 11-Oct & $12: 55: 29$ & 13:06:49 & $0: 11: 20$ & 5904 & 31.3 & 3 Lunch Hour, IVU not displaying suggested speed, on & 2165.55 & 11.44 & 28261100 & 4.27 \\
\hline DAY 4 & 0.09 & 11-Oct & 13:07:53 & 13:16:54 & 0:09:01 & 5884.2 & 39.2 & 2 IVU not displaying suggested speed, only cycle leng & 1915.12 & 12.72 & 24998300 & 4.75 \\
\hline DAY 4 & 0.1 & 1 11-Oct & $13: 20: 19$ & $13: 28: 14$ & 0:07:55 & 5903 & 44.7 & 7 IVU not displaying suggested speed, only cycle leng & 1769.06 & 13.38 & 23093600 & 5 \\
\hline DAY 4 & 0.11 & 1 11-Oct & $13: 29: 23$ & 13:37:51 & $0: 08: 28$ & 5896.5 & 41.8 & 8 IVU not displaying suggested speed, only cycle leng & 1905.11 & 13.47 & 24869600 & 5.04 \\
\hline DAY 4 & 0.12 & 211 -Oct & 13:41:10 & $13: 49: 22$ & 0:08:12 & 5914.1 & 43.3 & 3 IVU not displaying suggested speed, only cycle leng & 1983.94 & 14.49 & 25901100 & 5.41 \\
\hline DAY 4 & 0.13 & 3 11-Oct & 2:09:49 & $2: 19: 32$ & 0:09:43 & 5908 & 36.5 & 5 IVU not displaying suggested speed, only cycle leng & 1950.23 & 12.02 & 25453100 & 4.49 \\
\hline DAY 4 & 0.14 & 11-Oct & $2: 22: 50$ & $2: 30: 43$ & 0:07:53 & 5892.1 & 44.8 & 8 IVU not displaying suggested speed, only cycle leng & 1802.21 & 13.69 & 23527300 & 5.12 \\
\hline DAY 4 & 0.15 & 5 11-Oct & $2: 32: 26$ & 2:42:04 & 0:09:38 & 5974.8 & 37.2 & 2 IVU not displaying suggested speed, only cycle leng & 1964.99 & 12.21 & 25646700 & 4.56 \\
\hline DAY 4 & 0.16 & 5 11-Oct & 2:45:44 & $2: 54: 29$ & 0:08:45 & 5894.9 & 40.4 & 4 IVU not displaying suggested speed, only cycle leng & 1866.35 & 12.77 & 24361500 & 4.77 \\
\hline DAY 4 & 0.17 & $711-$ Oct & 2:55:42 & $3: 05: 00$ & 0:09:18 & 5884 & 38 & 8 IVU not displaying suggested speed, only cycle leng & 1867.86 & 12.02 & 24378800 & 4.49 \\
\hline DAY 4 & 0.18 & 3 11-Oct & 3:09:02 & $3: 18: 09$ & 0:09:07 & 5891.9 & 38.8 & 8 Two light cycles to get through at Riverside, IVU not & 1913.57 & 12.57 & 24977400 & 4.7 \\
\hline ON & 1 & 1 18-Oct & 9:26:20 & 9:35:43 & 0:09:23 & $3 \quad 5888.42$ & 37.65 & & 1901.45 & 12.16 & 24817400 & 4.53 \\
\hline ON & 2 & 2. 18 -Oct & 9:39:31 & $9: 48: 27$ & $0: 08: 56$ & $5 \quad 5889.85$ & 39.56 & & 1898.45 & 12.75 & 24780700 & 4.76 \\
\hline ON & 3 & 3 18-Oct & 9:49:52 & $9: 57: 50$ & $0: 07: 58$ & $\begin{array}{l}3886.69 \\
\end{array}$ & 44.33 & & 1614.38 & 12.16 & 21070600 & 4.53 \\
\hline ON & 4 & 18-Oct & 10:02:20 & 10:09:37 & 0:07:17 & $7 \quad 5896.33$ & 48.57 & & 1758.39 & 14.49 & 22957400 & 5.4 \\
\hline ON & 5 & 5 18-Oct & $10: 10: 37$ & 10:19:53 & $0: 09: 16$ & $5 \quad 5882.06$ & 38.09 & & 1969.69 & 12.75 & 25710600 & 4.76 \\
\hline ON & 6 & 5 18-Oct & 10:23:14 & 10:30:49 & 0:07:35 & 5889.06 & 46.59 & & 1762.89 & 13.95 & 23014700 & 5.2 \\
\hline ON & 7 & 7 18-Oct & $10: 48: 46$ & 10:58:17 & 0:09:31 & $1 \quad 5890.01$ & 37.13 & 3 Accident at Roydon & 2185.62 & 13.78 & 28532700 & 5.14 \\
\hline ON & 8 & 3 18-Oct & $11: 00: 53$ & 11:13:47 & $0: 12: 54$ & $4 \quad 5912.54$ & 27.5 & 5 Statistical outlier identified accident effects & 2308 & 10.73 & 30115800 & 4 \\
\hline ON & 9 & 18-Oct & 11:14:48 & 11:27:34 & $0: 12: 46$ & 5900.56 & 27.73 & 3 Accident at Roydon & 2396.49 & 11.26 & 31273900 & 4.2 \\
\hline ON & 10 & 18-Oct & 11:32:09 & 11:44:00 & 0:11:51 & $1 \quad 5898.24$ & 29.86 & 6 Statistical outlier identified accident effects & 2195.03 & 11.11 & 28644100 & 4.14 \\
\hline ON & 11 & 1 18 -Oct & 11:45:10 & 11:59:33 & $0: 14: 23$ & 5899.77 & 24.61 & 1 Accident at Roydon is cleared but the road is still col & 2440.29 & 10.18 & 31838300 & 3.79 \\
\hline ON & 12 & 2 18-Oct & 12:03:18 & $12: 17: 17$ & $0: 13: 59$ & $\begin{array}{l}7901.45 \\
\end{array}$ & 25.32 & 2 Lunch Hour & 2518.22 & 10.81 & 32859700 & 4.03 \\
\hline ON & 13 & 3 18-Oct & $12: 18: 29$ & $12: 28: 07$ & $0: 09: 38$ & 3 $\quad 5900.79$ & 36.75 & 5 Lunch Hour & 2022.19 & 12.59 & 26394800 & 4.7 \\
\hline ON & 14 & H 18 -Oct & $12: 33: 30$ & $12: 47: 51$ & $0: 14: 21$ & 5899.57 & 24.67 & 7 Lunch Hour & 2474.15 & 10.34 & 32281600 & 3.86 \\
\hline ON & 15 & 5 19-Oct & $9: 24: 35$ & $9: 32: 55$ & $0: 08: 20$ & 5878.57 & 42.33 & & 1634.17 & 11.77 & 21327600 & 4.39 \\
\hline ON & 16 & 5 19-Oct & $9: 37: 28$ & $9: 46: 56$ & $0: 09: 28$ & 5900.2 & 37.4 & & 1909.76 & 12.1 & 24925900 & 4.51 \\
\hline ON & 17 & 7 19-Oct & $9: 48: 18$ & 9:56:03 & 0:07:45 & 5886.28 & 45.57 & & 1669.51 & 12.93 & 21792200 & 4.82 \\
\hline ON & 18 & 3 19-Oct & $10: 00: 23$ & 10:09:24 & 0:09:01 & 5896.45 & 39.24 & & 1839.83 & 12.24 & 24013700 & 4.57 \\
\hline ON & 19 & 19-Oct & $10: 10: 30$ & 10:19:37 & 0:09:07 & 5886.04 & 38.74 & & 1811.62 & 11.92 & 23644300 & 4.45 \\
\hline ON & 20 & 19-Oct & 10:23:18 & 10:31:04 & $0: 07: 46$ & 5 $\quad 5882.46$ & 45.44 & & 1739.11 & 13.44 & 22702900 & 5.01 \\
\hline ON & 21 & 1 19-Oct & 10:45:09 & $10: 54: 45$ & $0: 09: 36$ & 5902.66 & 36.89 & & 2013.73 & 12.59 & 26284700 & 4.69 \\
\hline ON & 22 & 2. 19-Oct & $10: 58: 45$ & 11:08:11 & $0: 09: 26$ & 5890.73 & 37.47 & & 1894.79 & 12.05 & 24730300 & 4.49 \\
\hline ON & 23 & 3 19-Oct & 11:09:30 & $11: 18: 22$ & 0:08:52 & 5896.58 & 39.9 & & 1923.17 & 13.01 & 25104100 & 4.85 \\
\hline ON & 24 & 4 19-Oct & $11: 22: 44$ & 11:32:05 & $0: 09: 21$ & 5886.58 & 37.77 & & 1903.36 & 12.21 & 24842800 & 4.55 \\
\hline ON & 25 & 19-Oct & 11:36:41 & $11: 45: 59$ & $0: 09: 18$ & 5886.97 & 37.98 & & 1931.93 & 12.46 & 25216700 & 4.65 \\
\hline ON & 26 & 5 19-Oct & $11: 48: 50$ & 11:59:51 & 0:11:01 & 5901.45 & 32.14 & & 2009.1 & 10.94 & 26217200 & 4.08 \\
\hline ON & 27 & 7 19-Oct & $12: 56: 32$ & $13: 05: 54$ & $0: 09: 22$ & 5878.31 & 37.65 & 5 Lunch Hour & 1923.24 & 12.32 & 25102200 & 4.59 \\
\hline ON & 28 & 3 19-Oct & 13:09:49 & 13:21:35 & $0: 11: 46$ & 5939.14 & 30.28 & & 2289.6 & 11.68 & 29881000 & 4.35 \\
\hline ON & 29 & 19-Oct & $13: 22: 34$ & 13:31:11 & $0: 08: 37$ & 5898.25 & 41.07 & & 1931.09 & 13.45 & 25208800 & 5.02 \\
\hline ON & 30 & 19-Oct & $13: 34: 50$ & $13: 42: 57$ & 0:08:07 & 5919.74 & 43.76 & & 1861.04 & 13.76 & 24295400 & 5.13 \\
\hline ON & 31 & 1 19-Oct & 13:55:19 & $2: 04: 26$ & 0:09:07 & 5886.14 & 38.74 & & 1831.86 & 12.06 & 23908900 & 4.5 \\
\hline ON & 32 & 2 219-Oct & 2:08:13 & 2:17:47 & 0:09:34 & 5891.57 & 36.95 & & 1885.22 & 11.82 & 24604700 & 4.41 \\
\hline ON & 33 & 3 19-Oct & $2: 19: 39$ & $2: 28: 32$ & $0: 08: 53$ & 5894.06 & 39.81 & & 1885.9 & 12.74 & 24616700 & 4.75 \\
\hline ON & 34 & 19-Oct & $2: 33: 16$ & $2: 41: 32$ & $0: 08: 16$ & 5890.57 & 42.75 & & 1866.16 & 13.54 & 24362000 & 5.05 \\
\hline ON & 35 & 5 19-Oct & $2: 55: 13$ & $3: 04: 54$ & 0:09:41 & 5885.64 & 36.47 & & 2185.97 & 13.54 & 28536900 & 5.05 \\
\hline ON & 36 & 5 19-Oct & $3: 10: 59$ & $3: 21: 43$ & $0: 10: 44$ & 5897.89 & 32.97 & 7 Prince of whales and Riverside two light cyces each & 2034.93 & 11.38 & 26556400 & 4.24 \\
\hline ON & 37 & 7 19-Oct & 8:58:23 & 9:09:32 & 0:11:09 & 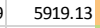 & 31.85 & & 2149.1 & 11.56 & 28047100 & 4.31 \\
\hline ON & 38 & 3 19-Oct & $9: 14: 10$ & 9:22:05 & 0:07:55 & 5894.26 & 44.67 & & 1682.93 & 12.75 & 21967500 & 4.76 \\
\hline ON & 39 & 19-Oct & $9: 23: 29$ & 9:31:04 & $0: 07: 35$ & 5886.07 & 46.57 & & 1558.22 & 12.33 & 20338400 & 4.6 \\
\hline ON & 40 & 19-Oct & 9:35:17 & $9: 44: 13$ & 0:08:56 & 5880.44 & 39.5 & & 1880.96 & 12.63 & 24552100 & 4.71 \\
\hline ON & 41 & 1. 19-Oct & $9: 54: 52$ & 10:04:39 & 0:09:47 & 5893.95 & 36.15 & & 2043.32 & 12.53 & 26670500 & 4.67 \\
\hline ON & 42 & 2 - 19 -Oct & 10:08:39 & $10: 17: 24$ & 0:08:45 & 5899.52 & 40.45 & & 1894.77 & 12.99 & 24733500 & 4.85 \\
\hline ON & 43 & 3 20-Oct & $10: 18: 30$ & $10: 27: 12$ & $0: 08: 42$ & 5881.14 & 40.56 & & 1806.98 & 12.46 & 23585900 & 4.65 \\
\hline ON & 44 & 20-Oct & 10:31:34 & $10: 40: 29$ & 0:08:55 & 5900.43 & 39.7 & & 1897.15 & 12.77 & 24763800 & 4.76 \\
\hline ON & 45 & $5 \quad 20$-Oct & $10: 55: 16$ & 11:03:51 & $0: 08: 35$ & 5890.29 & 41.17 & & 1900.13 & 13.28 & 24804400 & 4.95 \\
\hline ON & 46 & $5 \quad 20-0 c t$ & $11: 07: 12$ & $11: 17: 33$ & $0: 10: 21$ & 5887.53 & 34.13 & 3 Two light cycles at Prince of Whales to get through & 1921.32 & 11.14 & 25072600 & 4.15 \\
\hline ON & 47 & 20-Oct & 11:19:01 & 11:27:41 & $0: 08: 40$ & 5895.02 & 40.81 & & 1783.62 & 12.35 & 23280300 & 4.6 \\
\hline ON & 48 & $3 \quad 20$-Oct & 11:32:02 & 11:43:00 & $0: 10: 58$ & 5907.31 & 32.32 & & 2256.97 & 12.35 & 29458600 & 4.6 \\
\hline ON & 49 & 20-Oct & 11:56:21 & 12:05:16 & 0:08:55 & 5873.98 & 39.53 & & 1829.69 & 12.31 & 23881400 & 4.59 \\
\hline ON & 50 & 20-Oct & 12:09:32 & $12: 26: 48$ & $0: 17: 16$ & 5916.89 & 20.56 & 6 Lunch hour & 2864.75 & 9.95 & 37374400 & 3.71 \\
\hline ON & 51 & 1 20-Oct & $12: 27: 48$ & $12: 51: 58$ & $0: 24: 10$ & 5962.01 & 14.42 & 2 Lunch hour & 3594.22 & 8.7 & 46875800 & 3.24 \\
\hline ON & 52 & 20 -Oct & $12: 55: 27$ & 13:09:30 & 0:14:03 & $\begin{array}{l}3 \\
\end{array}$ & 25.27 & 7 Lunch hour & 2434.52 & 10.4 & 31764600 & 3.88 \\
\hline
\end{tabular}

Figure 23: Summary results with Average speeds and MOVES outputs b 


\section{Appendix C Additional Maps}

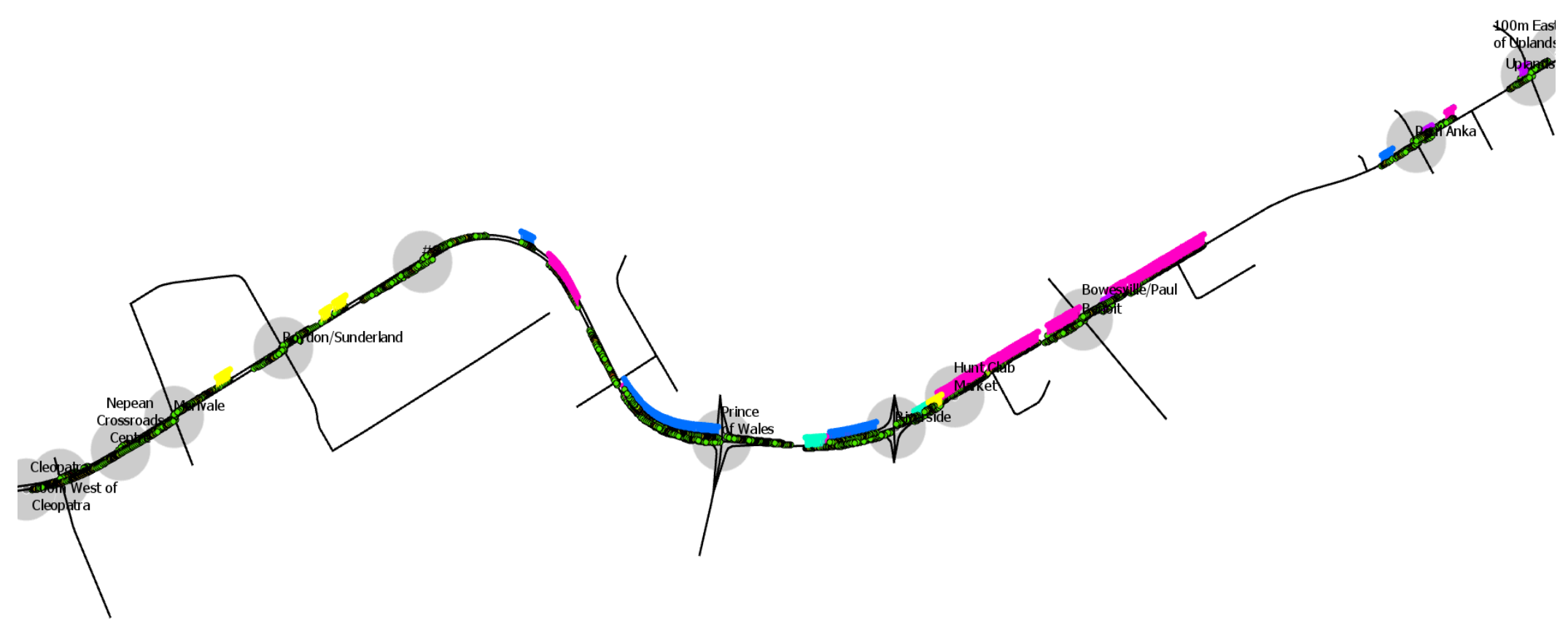

Figure 24: ArcMap GPS slow points evaluated for unusual traffic conditions 


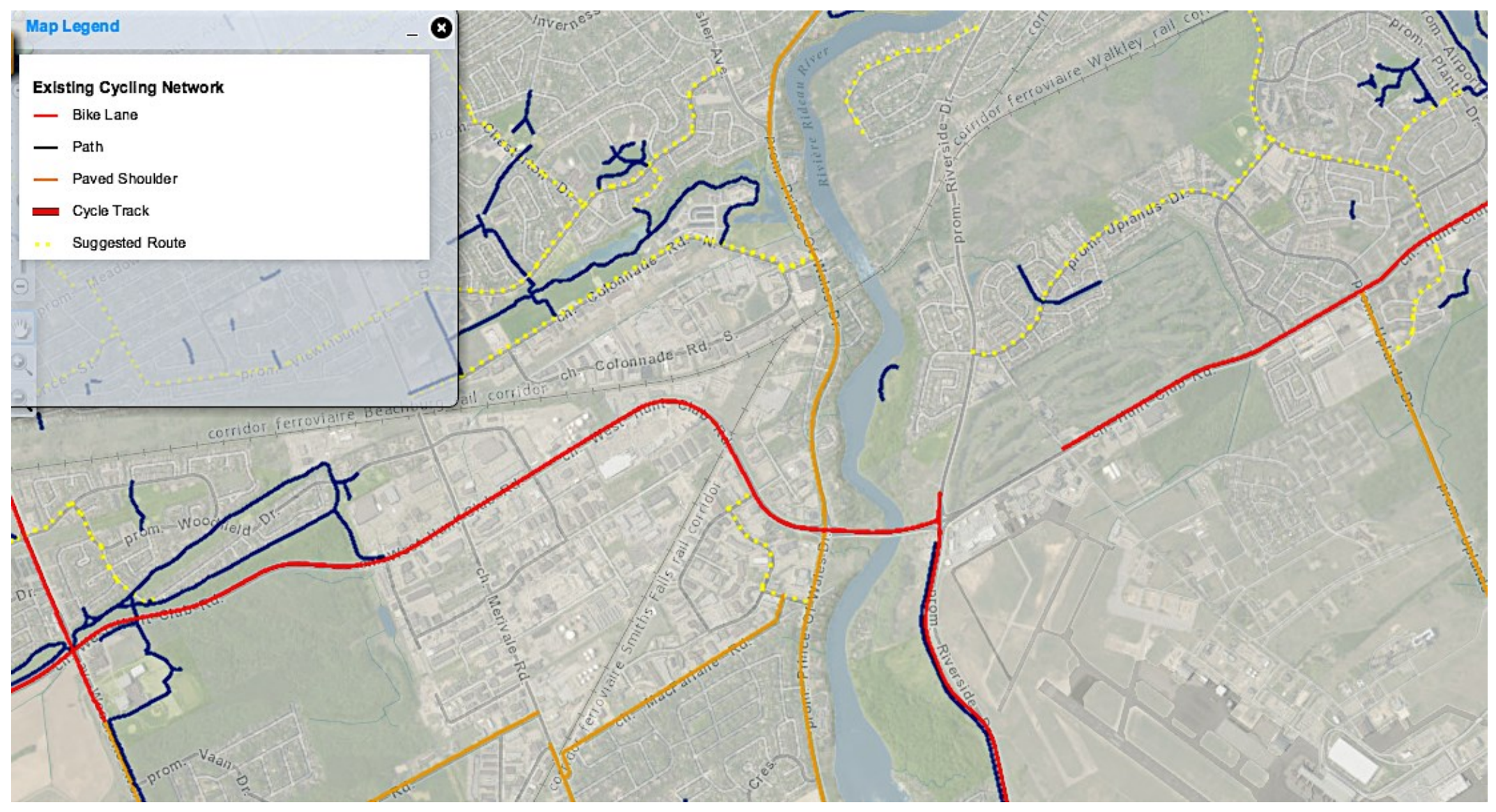

Figure 25: Cycling facilities on the Hunt Club Road Corridor [74] 


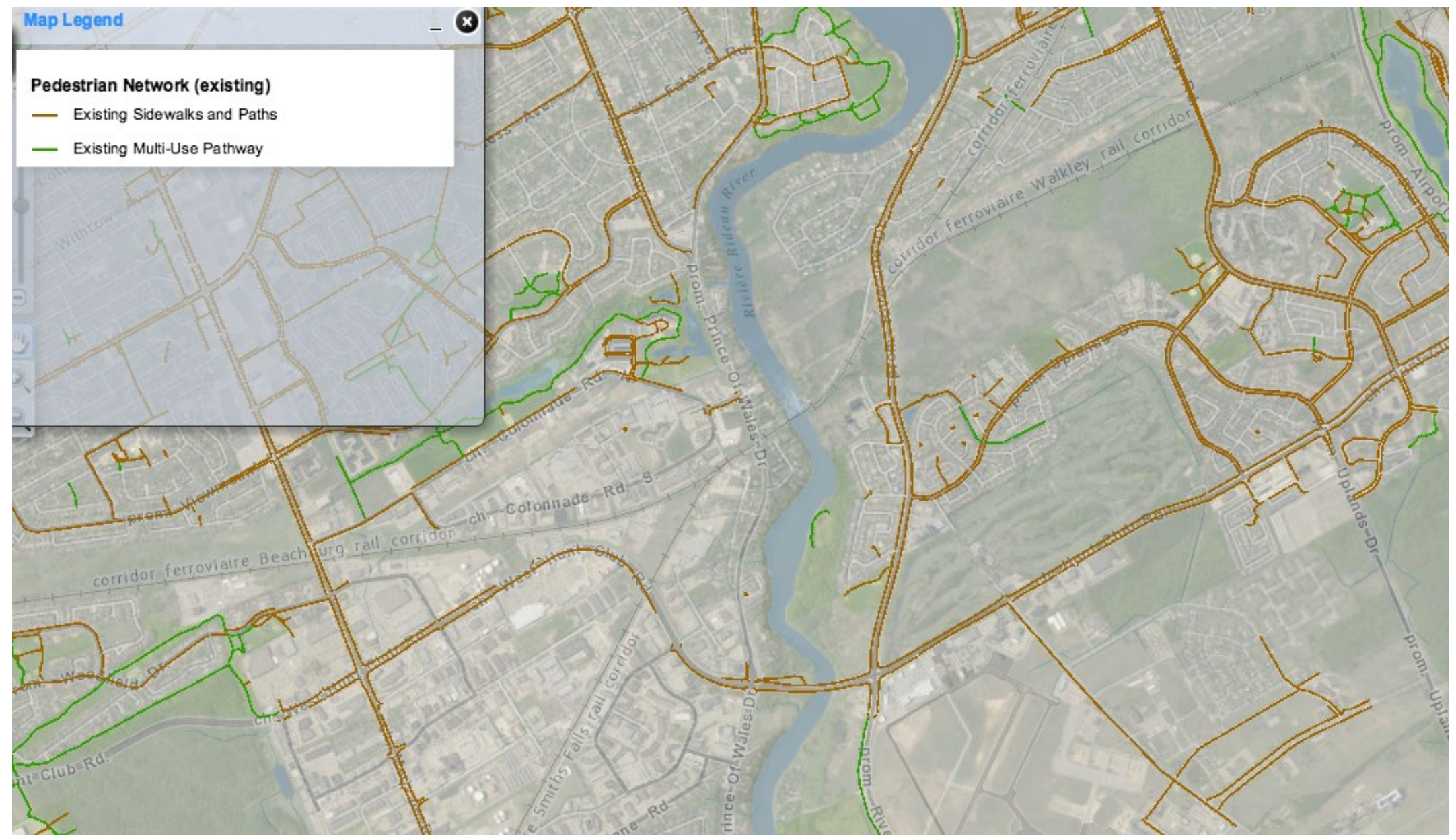

Figure 26: Pedestrian facilities on the Hunt Club Road Corridor [74] 


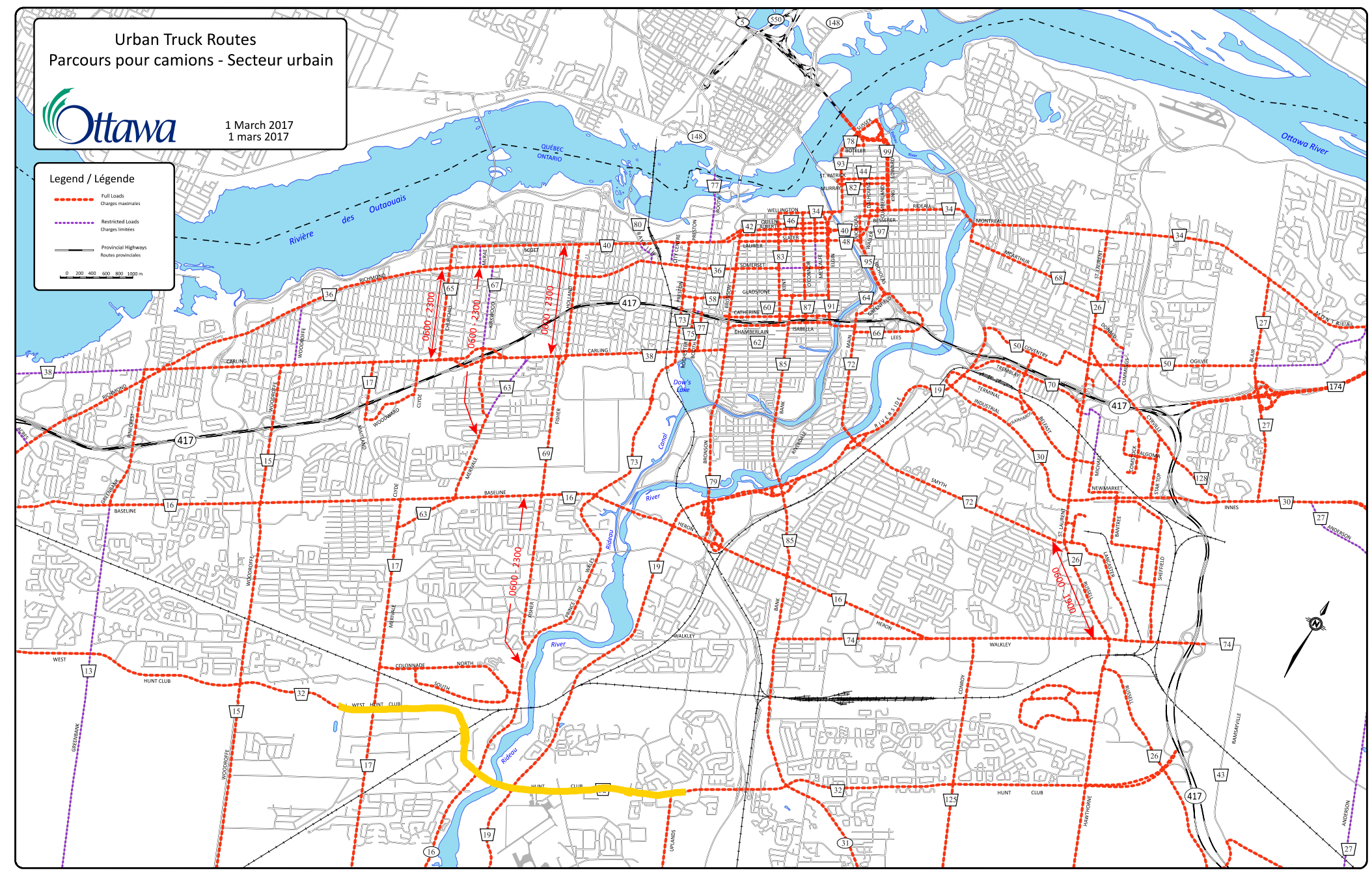

Figure 27: Ottawa urban trucking routes [75] 


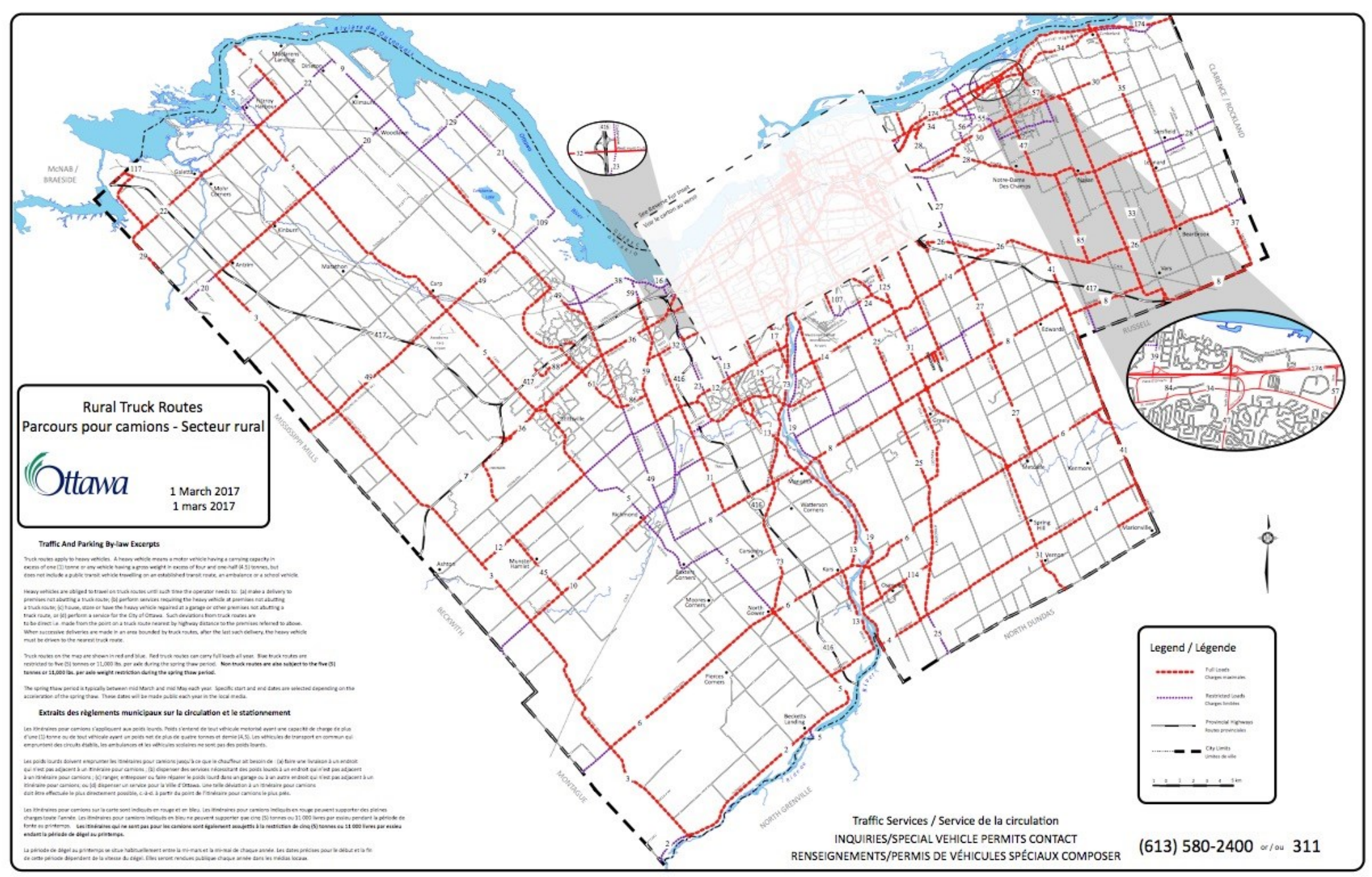

Figure 28: City of Ottawa rural trucking routes [76] 


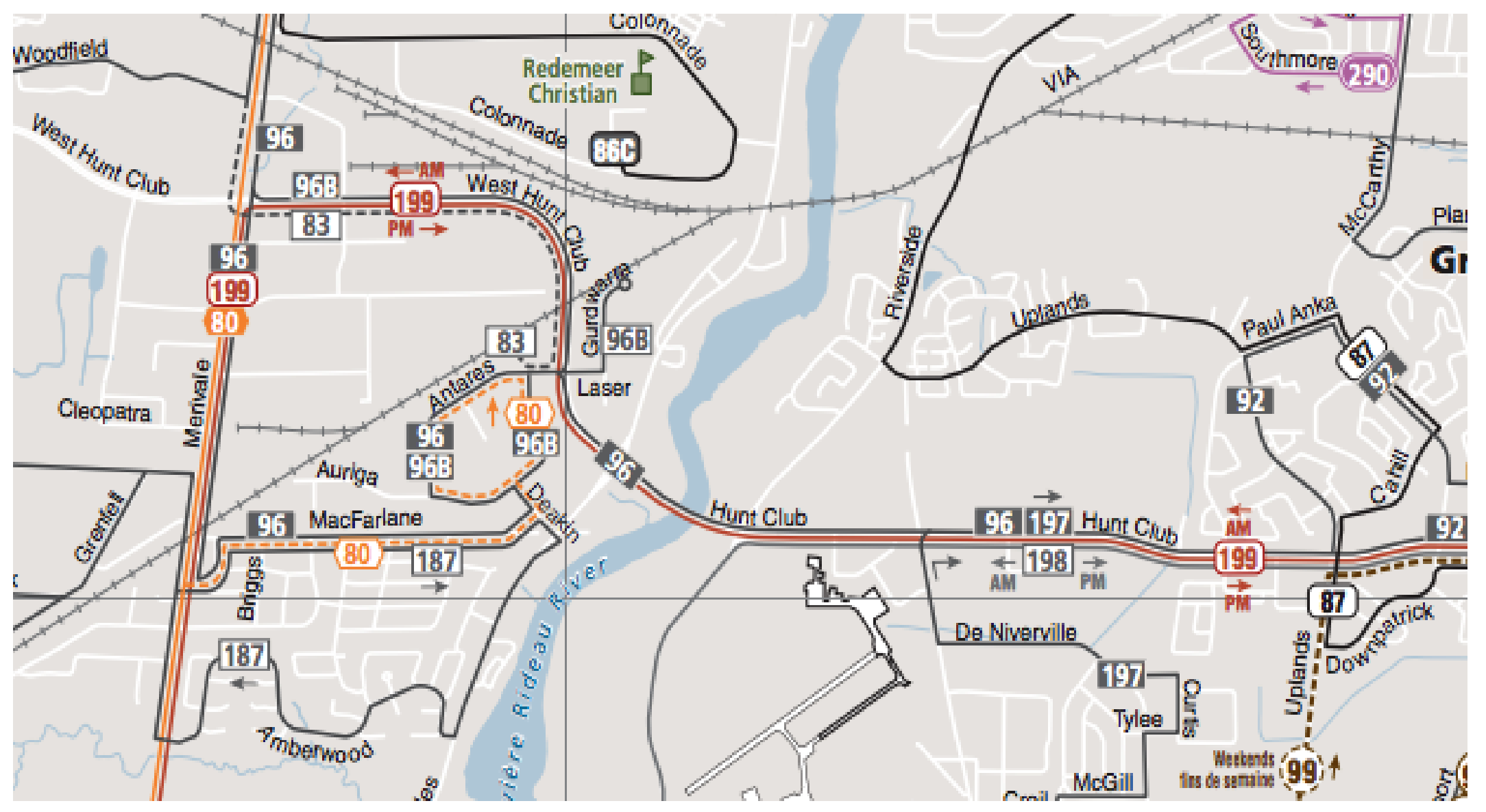

Figure 29: OC Transpo bus routes along the Hunt Club Road Corridor [77] 


\section{Appendix D Equipped Van}

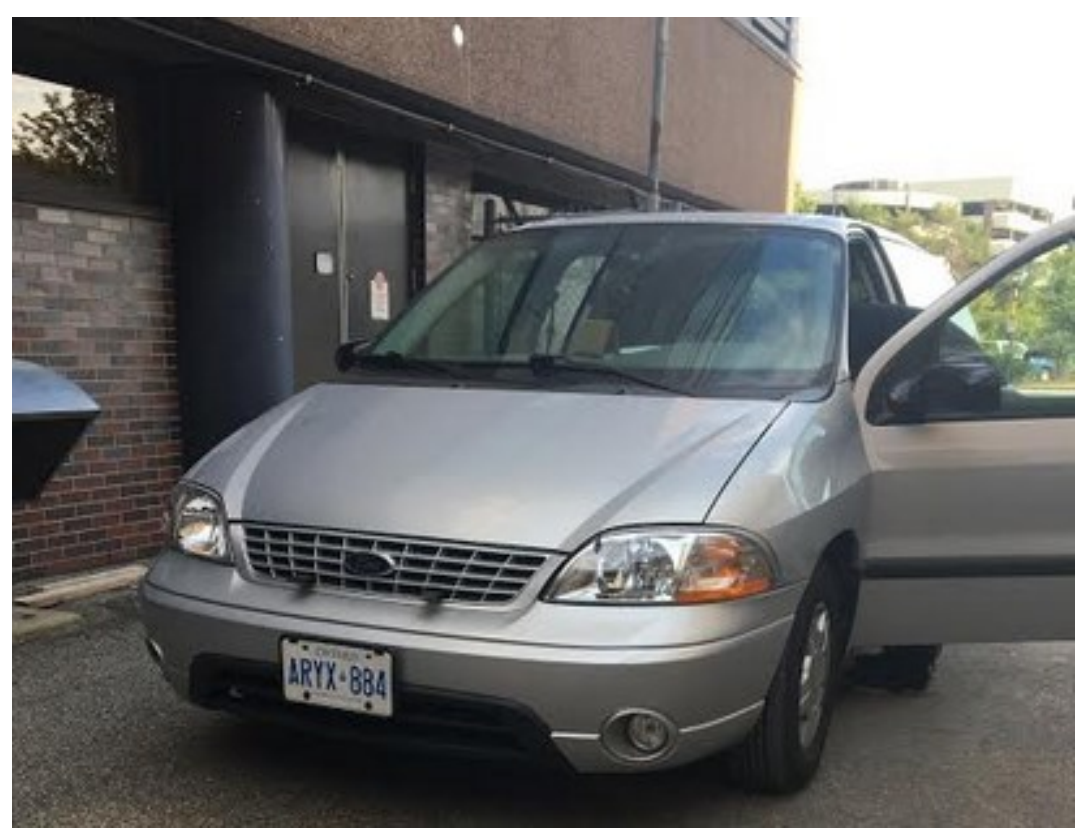

Figure 30: Carleton University test vehicle (the Van) 


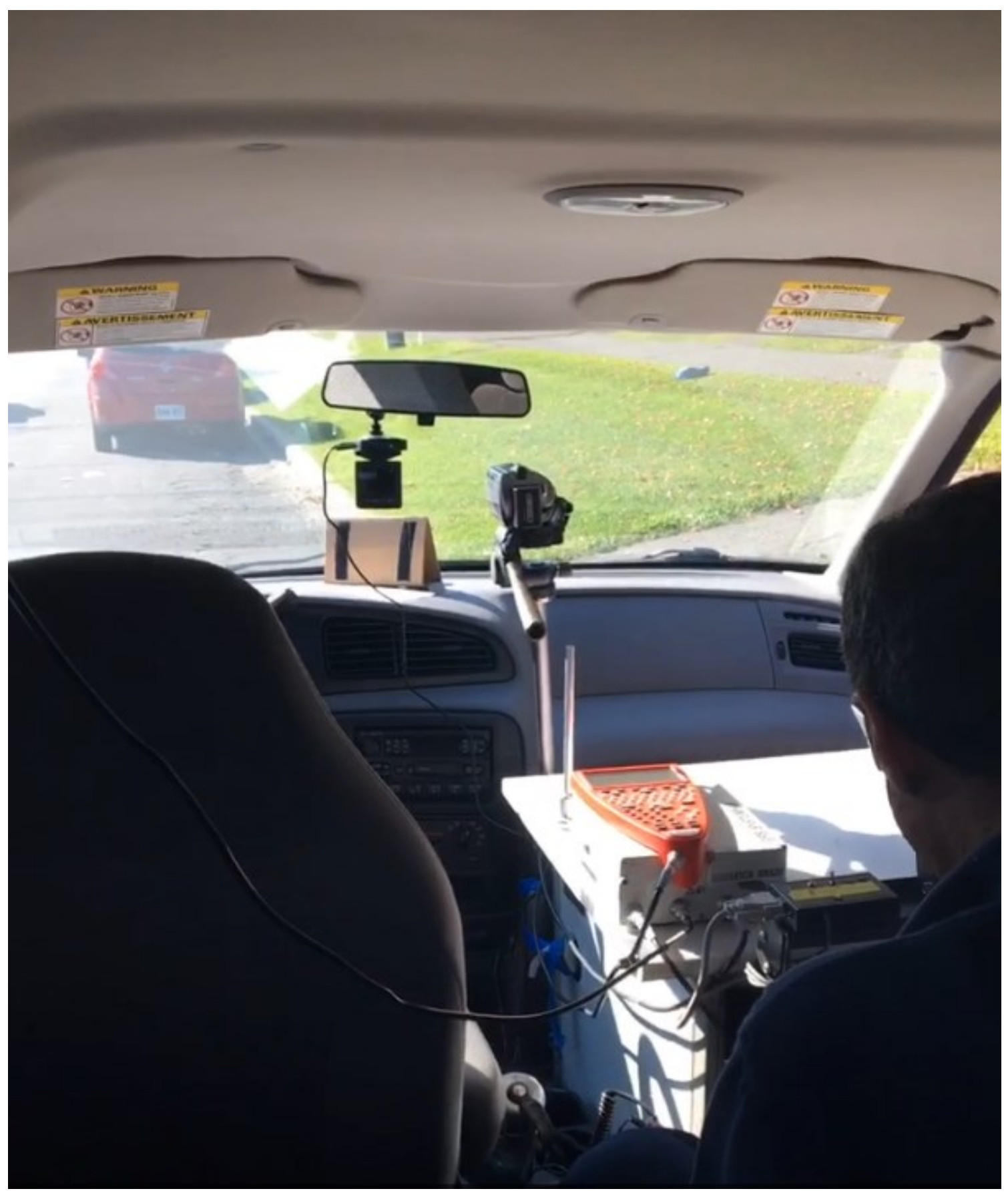

Figure 31: Configuration of equipment inside the Van 


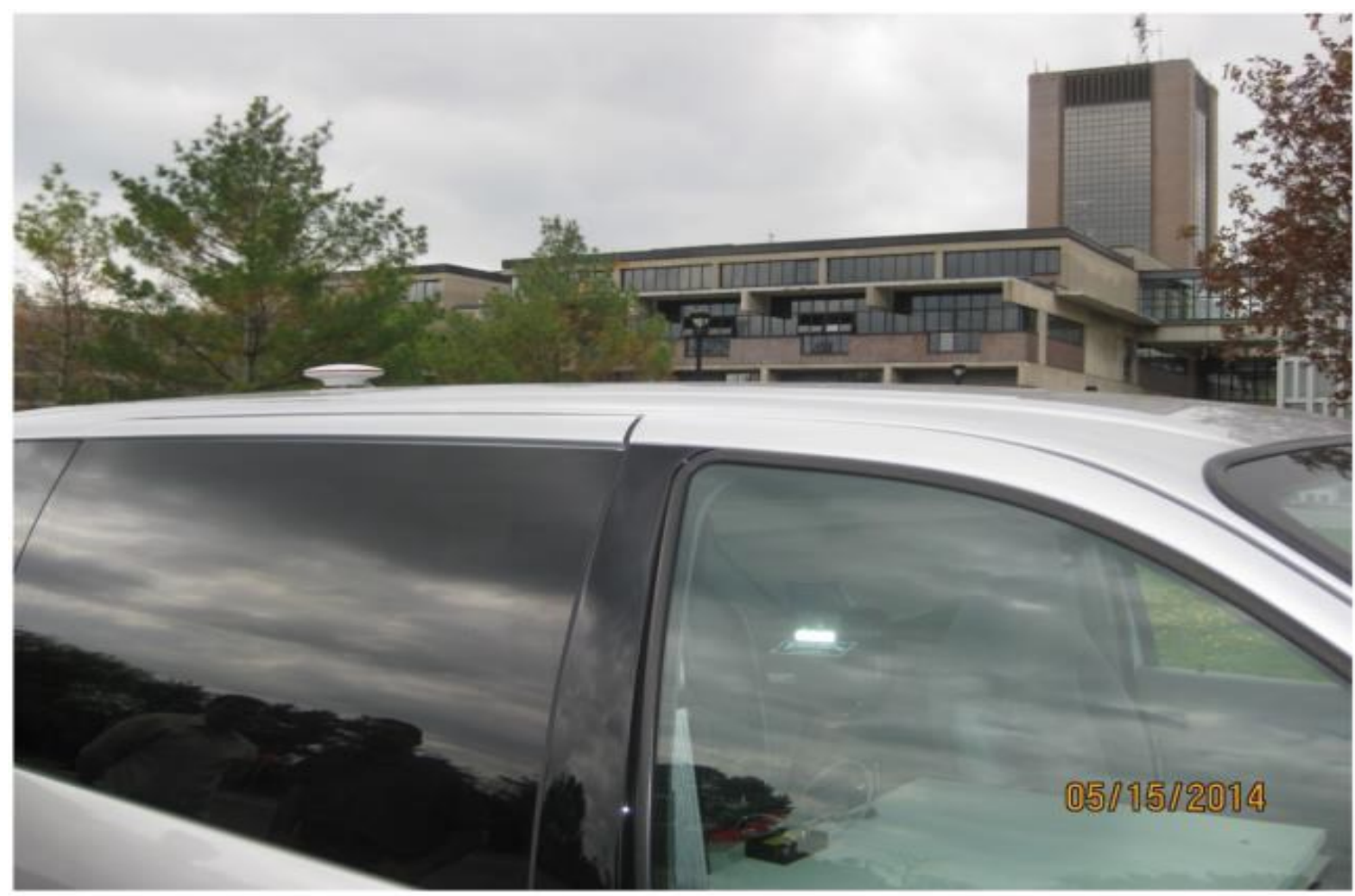

Figure 32: GPS antenna attached to the roof of the Van [78] 


\section{Appendix E R Scripts}

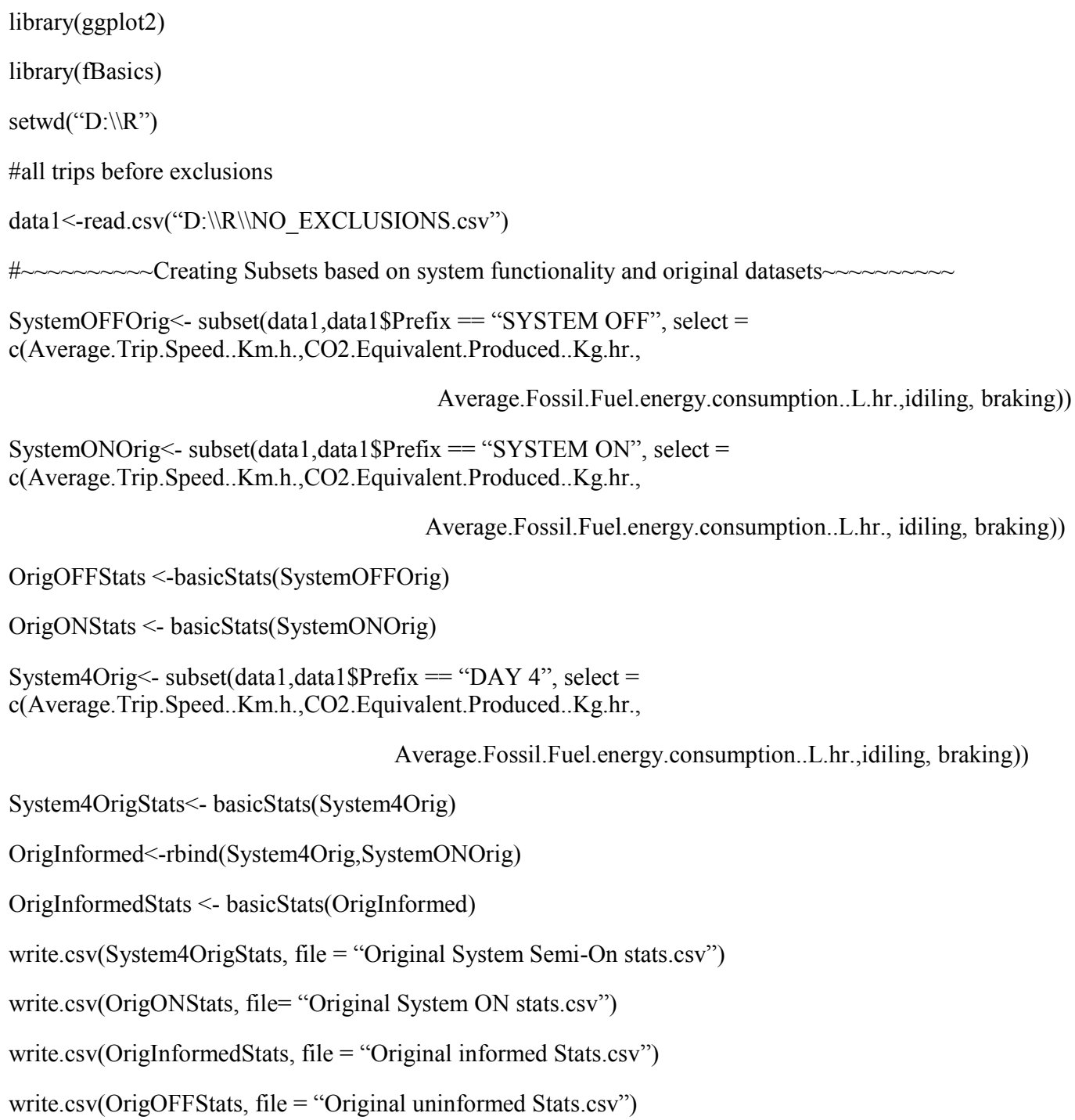


\# Creating Subsets based on system functionality

\#Days 1, 2 \& 3 System OFF

\#Days 5, 6 \& 7 System Fucntioning as intended with GLOSA recommendations

\#Without prefix for summary stats

SystemOFFAll<-subset $($ data, data\$Prefix $==$ "SYSTEM OFF",select $=$ c(Average.Trip.Speed..Km.h.,CO2.Equivalent.Produced..Kg.hr.,

Average.Fossil.Fuel.energy.consumption..L.hr.,idiling, braking,

S20, S50, S100, S150, AveGrade, LowSpeedCoasting..under25MPH., ModSpeedCoasting25to50MPH,

X0.3,X3to6, X6to9, X9to12, above12,

X0to3, X3to6.1, X6to9.1,X9to12.1, above12, X12to18, X18to24,

X18to24.1,

X24to30, over30, over50MPH..6, X6to12,X12to18.1,

X24to30.1, above30))

SystemONAll<-subset(data, data\$Prefix == "SYSTEM ON", select = c(Average.Trip.Speed..Km.h.,CO2.Equivalent.Produced..Kg.hr.,

Average.Fossil.Fuel.energy.consumption..L.hr.,idiling, braking,

S20, S50, S100, S150, AveGrade, LowSpeedCoasting..under25MPH., ModSpeedCoasting25to50MPH,

X0.3,X3to6, X6to9, X9to12, above12,

X0to3, X3to6.1, X6to9.1,X9to12.1, above12, X12to18, X18to24,

X24to30, over30, over50MPH..6, X6to12,X12to18.1, X18to24.1,

X24to30.1, above30))

\# with prefix

SystemOFFAllP<-subset(data, data\$Prefix == "SYSTEM OFF",select = c $($ Prefix, Average.Trip.Speed..Km.h.,CO2.Equivalent.Produced..Kg.hr.,

Average.Fossil.Fuel.energy.consumption..L.hr.,idiling, braking,

S20, S50, S100, S150, AveGrade, LowSpeedCoasting..under25MPH.,

ModSpeedCoasting25to50MPH,

X0.3, X3to6, X6to9, X9to12, above12,

X0to3, X3to6.1, X6to9.1,X9to12.1, above12, X12to18, X18to24,

X18to24.1,

X24to30, over30, over50MPH..6, X6to12,X12to18.1,

X24to30.1, above30))

SystemONAllP<-subset $($ data, data\$Prefix == "SYSTEM ON", select = c(Prefix,Average.Trip.Speed..Km.h.,CO2.Equivalent.Produced..Kg.hr., 
Average.Fossil.Fuel.energy.consumption..L.hr.,idiling, braking, S20, S50, S100, S150, AveGrade, LowSpeedCoasting..under25MPH., ModSpeedCoasting25to50MPH,

X0.3,X3to6, X6to9, X9to12, above12,

X18to24.1,

X0to3, X3to6.1, X6to9.1, X9to12.1, above12, X12to18, X18to24,

X24to30, over30, over50MPH..6, X6to12, X12to18.1,

X24to30.1, above30))

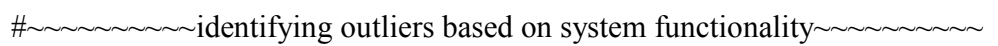

boxplot(data\$Average.Trip.Speed..Km.h. data\$Prefix, xlab = "System State”, ylab = "Average Trip Speed (km/h)",

main = "Outliers Based on Average Trip Speed and System State", col=c("red","green”))

\#no OFF speed outliers 3 on speed outliers

outlierSystemONSpeed <- boxplot.stats(SystemONAll\$Average.Trip.Speed..Km.h.)\$out

boxplot(data\$CO2.Equivalent.Produced..Kg.hr. data\$Prefix, xlab = "System State",

ylab = "Average CO2 Equivalent Produced $(\mathrm{Kg} / \mathrm{h}) "$,

main = "Outliers Based on Average CO2 Equivalent Produced and System State", col=c("red”,"green”))

boxplot(data\$Average.Fossil.Fuel.energy.consumption..L.hr. $\sim$ data\$Prefix, xlab = "System State",

ylab = "Average Fossil Fuel Consimption $(\mathrm{L} / \mathrm{h}) "$,

main $=$ "Outliers Based on Average Fossil Fuel Energy Consimption and System State", col=c("red","green"))

\#same as outliers for CO2E and FFEC

outlierSystemOFFFF <- boxplot.stats(SystemOFFAll\$Average.Fossil.Fuel.energy.consumption..L.hr.)\$out

outlierSystemONFF <- boxplot.stats(SystemONAll\$Average.Fossil.Fuel.energy.consumption..L.hr.)\$out

\# Removing Reasoned Outliers after video review

SystemOFFData $<-$ SystemOFFAll

SystemONData $<-$ SystemONAll[!(SystemONAll\$Average.Trip.Speed..Km.h.<30),]

SystemONAllP<-SystemONAllP[!(SystemONAllP\$Average.Trip.Speed..Km.h.<30),]



DataToSigTest<-rbind(SystemOFFData,SystemONData)

DataToSigTestP $<$-rbind(SystemOFFAllP,SystemONAllP)

\# Generating Statistical tables for all Data Sets

AllOFFStats $<$-basicStats(SystemOFFAll)

133 


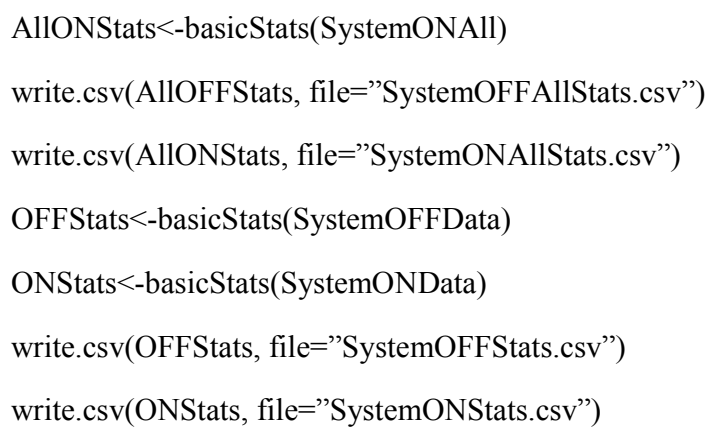


SpeedtTest2<-t.test(SystemONData\$Average.Trip.Speed..Km.h., SystemOFFData\$Average.Trip.Speed..Km.h., conf.int $=$ TRUE,

alternative $=$ "less")

\#significant

SpeedtTest3<-t.test(SystemONData\$Average.Trip.Speed..Km.h., SystemOFFData\$Average.Trip.Speed..Km.h., conf.int $=$ TRUE,

alternative $=$ "greater")

\#not significant

CO2EtTest<-t.test(SystemONData\$CO2.Equivalent.Produced..Kg.hr., SystemOFFData\$CO2.Equivalent.Produced..Kg.hr.)

FftTest<-t.test(SystemONData\$Average.Fossil.Fuel.energy.consumption..L.hr., SystemOFFData\$Average.Fossil.Fuel.energy.consumption..L.hr.)

Ttest<-c(SpeedtTest[[“p.value"]], CO2EtTest[[“p.value"]],FftTest[[“p.value"]])

\#coppied from all OFF/ON Stats

OFFMean $<-$

c(SystemOFFData\$Average.Trip.Speed..Km.h.,SystemOFFData\$CO2.Equivalent.Produced..Kg.hr.,SystemOFFData\$ Average.Fossil.Fuel.energy.consumption..L.hr.)

ONMean $<-$

c(SystemONData\$Average.Trip.Speed..Km.h.,SystemONData\$CO2.Equivalent.Produced..Kg.hr.,SystemONData\$Ave rage.Fossil.Fuel.energy.consumption..L.hr.)

PercentChange $<-(($ ONMean-OFFMean $) /$ OFFMean $) * 100$

Difference<-(ONMean-OFFMean)

OFFStandardDev $<-$

c(stdev(SystemOFFData\$Average.Trip.Speed..Km.h.),stdev(SystemOFFData\$CO2.Equivalent.Produced..Kg.hr.), stdev(SystemOFFData\$Average.Fossil.Fuel.energy.consumption..L.hr.))

ONStandardDev $<-$

c(stdev(SystemONData\$Average.Trip.Speed..Km.h.),stdev(SystemONData\$CO2.Equivalent.Produced..Kg.hr.), stdev(SystemONData\$Average.Fossil.Fuel.energy.consumption..L.hr.))

Pvalues<-data.frame(Variables, OFFShapiroPval, OFFMean, OFFStandardDev, ONShapiroPval, ONMean, ONStandardDev, PercentChange, Difference, Ttest)

\# significant: CO2.Equivalent.Produced..Kg.hr.,Average.Fossil.Fuel.energy.consumption..L.hr. S50

write.csv(Pvalues, file = "CorePvalues.csv") 


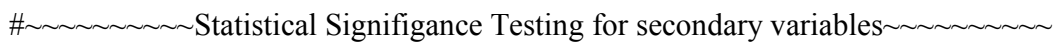

ONAveGrade<-shapiro.test(SystemONData\$AveGrade)

$\mathrm{Ni}<$-shapiro.test(SystemONData\$idiling)

Onb $<$-shapiro.test(SystemONData\$braking)

ON50<-shapiro.test(SystemONData\$S50)

Oni<-shapiro.test(SystemONData\$idiling)

Onb $<$-shapiro.test(SystemONData\$braking)

ON50<-shapiro.test(SystemONData\$S50)

ONLowSpeedCoasting..under25MPH.<-shapiro.test(SystemONData\$LowSpeedCoasting..under25MPH.)

ONX0.3<-shapiro.test(SystemONData\$X0.3)

ONX3to6<-shapiro.test(SystemONData\$X3to6)

ONX6to9<-shapiro.test(SystemONData\$X6to9)

ONX9to12<-shapiro.test(SystemONData\$X9to12)

Onabove12<-shapiro.test(SystemONData\$above12)

ONModSpeedCoasting25to50MPH<-shapiro.test(SystemONData\$ModSpeedCoasting25to50MPH)

ONX0to3<-shapiro.test(SystemONData\$X0to3)

ONX3to6.1<-shapiro.test(SystemONData\$X3to6.1)

ONX6to9.1<-shapiro.test(SystemONData\$X6to9.1)

ONX9to12.1<-shapiro.test(SystemONData\$X9to12.1)

Onabove12<-shapiro.test(SystemONData\$above12.1)

ONX12to18<-shapiro.test(SystemONData\$X12to18)

ONX18to24<-shapiro.test(SystemONData\$X18to24)

ONX24to30<-shapiro.test(SystemONData\$X24to30)

Onover30<-shapiro.test(SystemONData\$over30)

Onover50MPH..6<-shapiro.test(SystemONData\$over50MPH..6)

ONX6to12<-shapiro.test(SystemONData\$X6to12)

ONX12to18.1<-shapiro.test(SystemONData\$X12to18.1)

ONX18to24.1<-shapiro.test(SystemONData\$X18to24.1)

ONX24to30.1<-shapiro.test(SystemONData\$X24to30.1)

Onabove30<-shapiro.test(SystemONData\$above30)

ON<-c(ONAveGrade[[“p.value"]], Oni[[“p.value"]],Onb[[“p.value"]],ON50[[“p.value"]], ONLowSpeedCoasting..under25MPH. [["p.value"]], 
ONX0.3[[“p.value”]],ONX3to6[["p.value”]],ONX6to9[[“p.value”]], $\quad$ ONX9to12[[“p.value”]], Onabove12[["p.value"]],

ONModSpeedCoasting25to50MPH[[“p.value”]],ONX0to3[[“p.value”]], ONX3to6.1[[“p.value”]], ONX6to9.1[["p.value"]],

ONX9to12.1[["p.value"]], Onabove12[["p.value"]],ONX12to18[["p.value"]], ONX18to24[["p.value"]], ONX24to30[["p.value"]],

Onover30[[“p.value"]], Onover50MPH..6[[“p.value”]],ONX6to12[[“p.value”]], ONX12to18.1[[“p.value"]], ONX18to24.1[["p.value"]],

ONX24to30.1[[“p.value”]], Onabove30[[“p.value”]])

OFFAveGrade<-shapiro.test(SystemOFFData\$AveGrade)

OFFi<-shapiro.test(SystemOFFData\$idiling)

$\mathrm{OFFb}<$-shapiro.test $($ SystemOFFData\$braking)

OFF50<-shapiro.test(SystemOFFData\$S50)

OFFLowSpeedCoasting..under25MPH.<-shapiro.test(SystemOFFData\$LowSpeedCoasting..under25MPH.)

OFFX0.3<-shapiro.test(SystemOFFData\$X0.3)

OFFX3 to6<-shapiro.test(SystemOFFData\$X3to6)

OFFX6to9<-shapiro.test(SystemOFFData\$X6to9)

OFFX9to12<-shapiro.test(SystemOFFData\$X9to12)

OFFabove12<-shapiro.test(SystemOFFData\$above12)

OFFModSpeedCoasting25to50MPH<-shapiro.test(SystemOFFData\$ModSpeedCoasting25to50MPH)

OFFX0to3<-shapiro.test(SystemOFFData\$X0to3)

OFFX3 to6.1<-shapiro.test(SystemOFFData\$X3 to6.1)

OFFX6to9.1<-shapiro.test(SystemOFFData\$X6to9.1)

OFFX9to12.1<-shapiro.test(SystemOFFData\$X9to12.1)

OFFabove12<-shapiro.test(SystemOFFData\$above12.1)

OFFX12to18<-shapiro.test(SystemOFFData\$X12to18)

OFFX18to24<-shapiro.test(SystemOFFData\$X18to24)

OFFX24to30<-shapiro.test(SystemOFFData\$X24to30)

OFFover30<-shapiro.test(SystemOFFData\$over30)

OFFover50MPH..6<-shapiro.test(SystemOFFData\$over50MPH..6)

OFFX6to12<-shapiro.test(SystemOFFData\$X6to12)

OFFX12to18.1<-shapiro.test(SystemOFFData\$X12to18.1)

OFFX18to24.1<-shapiro.test(SystemOFFData\$X18to24.1)

OFFX24to30.1<-shapiro.test(SystemOFFData\$X24to30.1)

OFFabove30<-shapiro.test(SystemOFFData\$above30) 
OFF<-c(OFFAveGrade[[“p.value"]], OFFi[[“p.value"]], OFFb[[“p.value"]], OFF50[["p.value"]],

OFFLowSpeedCoasting..under25MPH.[["p.value”]],

OFFX0.3[[“p.value"]], OFFX3to6[[“p.value"]], OFFX6to9[[“p.value”]], OFFX9to12[[“p.value"]], OFFabove12[[“p.value”]],

OFFModSpeedCoasting25to50MPH[[“p.value"]], OFFX0to3[[“p.value"]], OFFX3to6.1[[“p.value"]], OFFX6to9.1[[“p.value"]],

OFFX9to12.1[[“p.value"]], OFFabove12[[“p.value”]], OFFX12to18[["p.value"]], OFFX18to24[["p.value"]], OFFX24to30[[“p.value”]],

OFFover30[[“p.value”]], OFFover50MPH..6[[“p.value”]], OFFX6to12[[“p.value”]], OFFX12to18.1[[“p.value"]], OFFX18to24.1[[“p.value"]],

OFFX24to30.1[[“p.value”]], OFFabove30[[“p.value”]])

names<-c(“AveGrade",’Idiling”, "Braking”, "Stops", "LowSpeedCoasting..under25MPH.," "X0.3”,,X3to6”,"X6to9”, "X9to12", "above12","ModSpeedCoasting25to50MPH”,

"X0to3", "X3to6.1”, “X6to9.1”, "X9to12.1”, "above12”, "X12to18”, “X18to24”, "X24to30”, “over30", “over50MPH..6","X6to12",

“X12to18.1”, “X18to24.1”, "X24to30.1”, “above30”)

ONMeanAll<-

c(mean(SystemONData\$AveGrade),mean(SystemONData\$idiling),mean(SystemONData\$braking), mean(SystemOND ata\$S50),

mean(SystemONData\$LowSpeedCoasting..under25MPH.),mean(SystemONData\$X0.3),mean(SystemONData\$X3to6) ,mean(SystemONData\$X6to9),

mean(SystemONData\$X9to12),mean(SystemONData\$above12), mean(SystemONData\$ModSpeedCoasting25to50MP $\mathrm{H})$, mean(SystemONData $\$ \mathrm{X} 0$ to3),

mean(SystemONData\$X3to6.1),mean(SystemONData\$X6to9.1),mean(SystemONData\$X9to12.1),mean(SystemONDa ta\$above12.1), mean(SystemONData\$X12to18),

mean(SystemONData\$X18to24), mean(SystemONData\$24to30), mean(SystemONData\$over30), mean(SystemONDat a\$over50MPH..6),mean(SystemONData\$X6to12),

mean(SystemONData\$X12to18.1), mean(SystemONData\$X18to24.1),

mean(SystemONData\$X24to30.1), mean(SystemONData\$above30))

OFFMeanAll<-

c(mean(SystemOFFData\$AveGrade), mean(SystemOFFData\$idiling), mean(SystemOFFData\$braking), mean(SystemOF FData\$S50),

mean(SystemOFFData\$LowSpeedCoasting..under25MPH.),mean(SystemOFFData\$X0.3), mean(SystemOFFData\$X3t o6), mean(SystemOFFData\$X6to9), 
mean(SystemOFFData\$X9to12),mean(SystemOFFData\$above12), mean(SystemOFFData\$ModSpeedCoasting25to50 $\mathrm{MPH})$,mean(SystemOFFData\$X0to3),

mean(SystemOFFData\$X3to6.1), mean(SystemOFFData\$X6to9.1), mean(SystemOFFData\$X9to12.1), mean(SystemOF FData\$above12.1),

mean(SystemOFFData\$X12to18), mean(SystemOFFData\$X18to24), mean(SystemOFFData \$X24to30),mean(SystemOF FData\$over30),

mean(SystemOFFData\$over50MPH..6),mean(SystemOFFData\$X6to12),mean(SystemOFFData\$X12to18.1),mean(Sys temOFFData $\$$ X18to24.1),

mean(SystemOFFData\$X24to30.1),mean(SystemOFFData\$above30))

ONMedianAll<-

c(median(SystemONData\$AveGrade),median(SystemONData\$idiling),median(SystemONData\$braking),median(Syste mONData\$S50),

median(SystemONData\$LowSpeedCoasting..under25MPH.),median(SystemONData\$X0.3),median(SystemONData\$ X3to6),median(SystemONData\$X6to9),

median(SystemONData\$X9to12), median(SystemONData\$above12), median(SystemONData\$ModSpeedCoasting25to5 0MPH), median(SystemONData\$X0to3),

median(SystemONData\$X3to6.1),median(SystemONData\$X6to9.1),median(SystemONData\$X9to12.1),median(Syste mONData\$above12.1),

median(SystemONData\$X12to18),median(SystemONData\$X18to24),median(SystemONData\$X24to30), median(Syste mONData\$over30),

median(SystemONData\$over50MPH..6),median(SystemONData\$X6to12),median(SystemONData\$X12to18.1),media $\mathrm{n}($ SystemONData\$X18to24.1),

median(SystemONData\$X24to30.1),median(SystemONData\$above30))

OFFMedianAll<-

c(median(SystemOFFData\$AveGrade),median(SystemOFFData\$idiling),median(SystemOFFData\$braking), median(Sy stemOFFData\$S50),

median(SystemOFFData\$LowSpeedCoasting..under25MPH.),median(SystemOFFData\$X0.3),median(SystemOFFData \$X3to6), median(SystemOFFData\$X6to9),

median(SystemOFFData\$X9to12), median(SystemOFFData\$above12),median(SystemOFFData\$ModSpeedCoasting25 to50MPH), median(SystemOFFData\$X0to3),

median(SystemOFFData\$X3 to6.1),median(SystemOFFData\$X6to9.1), median(SystemOFFData\$X9to12.1), median(Sy stemOFFData\$above12.1), 
median(SystemOFFData\$X12to18), median(SystemOFFData\$X18to24),median(SystemOFFData\$X24to30), median(Sy stemOFFData\$over30),

median(SystemOFFData\$over50MPH..6),median(SystemOFFData\$X6to12), median(SystemOFFData\$X12to18.1),med ian(SystemOFFData\$X18to24.1),

median(SystemOFFData\$24to30.1),median(SystemOFFData\$above30))

PercentChangeAll<-(((ONMeanAll-OFFMeanAll)/OFFMeanAll)*100)

DifferenceAll<-(ONMeanAll-OFFMeanAll)

wOFFAveGrade<-wilcox.test(SystemOFFData\$AveGrade, SystemONData\$AveGrade)

wOFFIdiling<-wilcox.test(SystemOFFData\$idiling, SystemONData\$idiling)

wOFFBraking<-wilcox.test(SystemOFFData\$braking, SystemONData\$braking)

wOFFStops<-wilcox.test(SystemOFFData\$S50, SystemONData\$S50)

wOFFLowSpeedCoasting..under25MPH.<-wilcox.test(SystemOFFData\$LowSpeedCoasting..under25MPH., SystemONData\$LowSpeedCoasting..under25MPH.)

wOFFX0.3<-wilcox.test(SystemOFFData\$X0.3, SystemONData\$X0.3)

wOFFX3to6<-wilcox.test(SystemOFFData\$X3to6, SystemONData\$X3to6)

wOFFX6to9<-wilcox.test(SystemOFFData\$X6to9, SystemONData\$X6to9)

wOFFX9to12<-wilcox.test(SystemOFFData\$X9to12, SystemONData\$X9to12)

wOFFabove12<-wilcox.test(SystemOFFData\$above12, SystemONData\$above12)

wOFFModSpeedCoasting25to50MPH<-wilcox.test(SystemOFFData\$ModSpeedCoasting25to50MPH, SystemONData\$ModSpeedCoasting25to50MPH)

wOFFX0to3<-wilcox.test(SystemOFFData\$X0to3, SystemONData\$X0to3)

wOFFX3to6.1<-wilcox.test(SystemOFFData $\$ X 3$ to6.1, SystemONData $\$$ X3to6.1)

wOFFX6to9.1<-wilcox.test(SystemOFFData\$X6to9.1, SystemONData\$X6to9.1)

wOFFX9to12.1<-wilcox.test(SystemOFFData\$X9to12.1, SystemONData\$X9to12.1)

wOFFabove12<-wilcox.test(SystemOFFData\$above12.1, SystemONData\$above12.1)

wOFFX12to18<-wilcox.test(SystemOFFData\$X12to18, SystemONData\$X12to18)

wOFFX18to24<-wilcox.test(SystemOFFData\$X18to24, SystemONData\$X18to24)

wOFFX24to30<-wilcox.test(SystemOFFData\$X24to30, SystemONData\$X24to30)

wOFFover30<-wilcox.test(SystemOFFData\$over30, SystemONData\$over30)

wOFFover50MPH..6<-wilcox.test(SystemOFFData\$over50MPH..6, SystemONData\$over50MPH..6)

wOFFX6to12<-wilcox.test(SystemOFFData\$X6to12, SystemONData\$X6to12) 
wOFFX12to18.1<-wilcox.test(SystemOFFData\$X12to18.1, SystemONData\$X12to18.1)

wOFFX18to24.1<-wilcox.test(SystemOFFData\$X18to24.1, SystemONData\$X18to24.1)

wOFFX24to30.1<-wilcox.test(SystemOFFData\$X24to30.1, SystemONData\$X24to30.1)

wOFFabove30<-wilcox.test(SystemOFFData\$above30, SystemONData\$above30)

tOFFAveGrade<-t.test(SystemOFFData $\$$ AveGrade, SystemONData\$AveGrade)

tOFFIdiling<-t.test(SystemOFFData\$idiling, SystemONData\$idiling)

tOFFIdiling2<-t.test(SystemOFFData\$idiling, SystemONData\$idiling, conf.int = TRUE, alternative = "less")

tOFFIdiling3<-t.test(SystemOFFData\$idiling, SystemONData\$idiling, conf.int = TRUE, alternative = "greater”)

tOFFBraking<-t.test(SystemOFFData\$braking, SystemONData\$braking)

tOFFStops<-t.test(SystemOFFData\$S50, SystemONData\$S50)

tOFFStops2<-t.test(SystemOFFData\$S50, SystemONData\$S50, conf.int = TRUE, alternative = "less")

tOFFStops3<-t.test(SystemOFFData\$S50, SystemONData\$S50, conf.int = TRUE, alternative = “greater")

tOFFLowSpeedCoasting..under25MPH.<-t.test(SystemOFFData\$LowSpeedCoasting..under25MPH., SystemONData\$LowSpeedCoasting..under25MPH.)

tOFFX0.3<-t.test(SystemOFFData\$X0.3, SystemONData\$X0.3)

tOFFX3to6<-t.test(SystemOFFData\$X3to6, SystemONData\$X3to6)

tOFFX6to9<-t.test(SystemOFFData\$X6to9, SystemONData\$X6to9)

tOFFX9to12<-t.test(SystemOFFData\$X9to12, SystemONData\$X9to12)

tOFFabove12<-t.test(SystemOFFData\$above12, SystemONData\$above12)

tOFFModSpeedCoasting25to50MPH<-t.test(SystemOFFData\$ModSpeedCoasting25to50MPH, SystemONData\$ModSpeedCoasting25to50MPH)

tOFFX0to3<-t.test(SystemOFFData\$X0to3, SystemONData\$X0to3)

tOFFX3 to6.1<-t.test(SystemOFFData\$X3to6.1, SystemONData\$X3to6.1)

tOFFX6to9.1<-t.test(SystemOFFData\$X6to9.1, SystemONData\$X6to9.1)

tOFFX9to12.1<-t.test(SystemOFFData\$X9to12.1, SystemONData\$X9to12.1)

tOFFabove12<-t.test(SystemOFFData\$above12.1, SystemONData\$above12.1)

tOFFX12to18<-t.test(SystemOFFData\$X12to18, SystemONData\$X12to18)

tOFFX18to24<-t.test(SystemOFFData\$X18to24, SystemONData\$X18to24)

tOFFX24to30<-t.test(SystemOFFData\$X24to30, SystemONData\$X24to30)

tOFFover30<-t.test(SystemOFFData\$over30, SystemONData\$over30)

tOFFover50MPH..6<-t.test(SystemOFFData\$over50MPH..6, SystemONData\$over50MPH..6) 
tOFFX6to12<-t.test(SystemOFFData\$X6to12, SystemONData\$X6to12)

tOFFX12to18.1<-t.test(SystemOFFData\$X12to18.1, SystemONData\$X12to18.1)

tOFFX18to24.1<-t.test(SystemOFFData\$X18to24.1, SystemONData\$X18to24.1)

tOFFX24to30.1<-t.test(SystemOFFData\$X24to30.1, SystemONData\$X24to30.1)

tOFFabove30<-t.test(SystemOFFData\$above30, SystemONData\$above30)

Allt<-c(tOFFAveGrade[[“p.value"]], tOFFIdiling[[“p.value"]], tOFFBraking[[“p.value"]], tOFFStops[[“p.value"]],

tOFFLowSpeedCoasting..under25MPH.[["p.value"]], tOFFX0.3[[“p.value”]],

tOFFX3 to6[[“p.value"]],tOFFX6to9[[“p.value"]],

tOFFX9to12[["p.value"]],

tOFFabove12[["p.value"]],tOFFModSpeedCoasting25to50MPH[[“p.value"]],tOFFX0to3[["p.value"]],

tOFFX3to6.1[[“p.value"]], tOFFX6to9.1[["p.value"]], tOFFX9to12.1[["p.value"]], tOFFabove12[[“p.value"]],tOFFX12to18[[“p.value”]],

tOFFX18to24[[“p.value"]], tOFFX24to30[[“p.value”]], tOFFover30[["p.value”]], tOFFover50MPH..6[["p.value"]],tOFFX6to12[[“p.value"]],

tOFFX12to18.1[["p.value"]], tOFFX18to24.1[["p.value"]], tOFFX24to30.1[["p.value"]], tOFFabove30[[“p.value"]])

Allw<-c(wOFFAveGrade[["p.value"]], wOFFIdiling[[“p.value"]], wOFFBraking[[“p.value"]], wOFFStops[["p.value"]],

wOFFLowSpeedCoasting..under25MPH.[["p.value"]], wOFFX0.3[[“p.value"]], wOFFX3to6[[“p.value"]], wOFFX6to9[["p.value"]],

wOFFX9to12[[“p.value”]], wOFFabove12[[“p.value”]], wOFFModSpeedCoasting25to50MPH[[“p.value"]], wOFFX0to3[[“p.value"]],

wOFFX3to6.1[[“p.value"]], wOFFX6to9.1[[“p.value"]], wOFFX9to12.1[["p.value"]], wOFFabove12[["p.value"]], wOFFX12to18[[“p.value"]],

wOFFX18to24[[“p.value"]], wOFFX24to30[["p.value"]], wOFFover30[[“p.value"]], wOFFover50MPH..6[[“p.value”]], wOFFX6to12[[“p.value”]],

wOFFX12to18.1[[“p.value"]], wOFFX18to24.1[[“p.value”]], wOFFX24to30.1[["p.value”]], wOFFabove30[[“p.value"]])

Others<-data.frame(names,OFF,OFFMeanAll, OFFMedianAll, ON, ONMeanAll, ONMedianAll, PercentChangeAll, DifferenceAll, Allt, Allw)

write.csv(Others, file $=$ "Suplementary p-values.csv")

\#significant: LowSpeedCoasting..under25MPH., ModSpeedCoasting25to50MPH, X9to12.1, over50MPH..6

\# Primary + Significant variables only

SysOFF<-subset(SystemOFFAllP, select = c(Prefix, Average.Trip.Speed..Km.h., CO2.Equivalent.Produced..Kg.hr., Average.Fossil.Fuel.energy.consumption..L.hr., 
idiling, braking, S50, LowSpeedCoasting..under25MPH., ModSpeedCoasting25to50MPH,X9to12.1))

SysON<-subset(SystemONAllP, select = c(Prefix, Average.Trip.Speed..Km.h., CO2.Equivalent.Produced..Kg.hr., Average.Fossil.Fuel.energy.consumption..L.hr.,

idiling, braking, S50, LowSpeedCoasting..under25MPH., ModSpeedCoasting25to50MPH,X9to12.1))

SysAll<-rbind(SysOFF,SysON)

\# us $\operatorname{lm}$ to fit a regression line

fit <-lm( CO2.Equivalent.Produced..Kg.hr. idiling + braking +S50+ LowSpeedCoasting..under25MPH. + ModSpeedCoasting25to50MPH, data=SysAll)

summary(fit)

SysAllR2<-summary(fit)\$r.squared

fit $2<-\operatorname{lm}$ ( CO2.Equivalent.Produced..Kg.hr. braking + LowSpeedCoasting..under25MPH. + ModSpeedCoasting25to50MPH, data=SysAll)

summary(fit2)

fit $2<-\operatorname{lm}($ CO2.Equivalent.Produced..Kg.hr. Average.Trip.Speed..Km.h.+ braking + LowSpeedCoasting..under25MPH. + ModSpeedCoasting25to50MPH, data=SysAll)

summary(fit2)

basicstats(SystemOFFData\$Average.Trip.Speed..Km.h.)

summary(SystemONData\$Average.Trip.Speed..Km.h.)

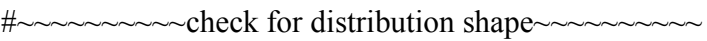

hist(SystemOFFData\$Average.Trip.Speed..Km.h., col="red", main = "System OFF”, breaks = $\mathrm{c}(28,30,32,34,36,38,40,42,44,46,48,50,52,54)$,

$\mathrm{xlab}=$ "Average trip speed $(\mathrm{Km} / \mathrm{h}) ")$

hist(SystemONData\$Average.Trip.Speed..Km.h., col="green", main = "System ON", breaks = $\mathrm{c}(28,30,32,34,36,38,40,42,44,46,48,50,52,54)$,

$\mathrm{xlab}=$ "Average trip speed $(\mathrm{Km} / \mathrm{h}) ")$

hist(SystemOFFData\$CO2.Equivalent.Produced..Kg.hr., col="red", main = "System OFF", breaks $=c(10,11,12,13,14,15,16,17,18), x l a b=$ "Average $\mathrm{CO} 2$ equivalent emissions produced $(\mathrm{Kg} / \mathrm{h}) ")$

hist(SystemONData\$CO2.Equivalent.Produced..Kg.hr., breaks= c(10,11,12,13,14,15,16,17,18), col="green", main = "System ON",

$\mathrm{xlab}=$ "Average $\mathrm{CO} 2$ equivalent emissions produced $(\mathrm{Kg} / \mathrm{h})$ ") 
hist(SystemOFFData\$Average.Fossil.Fuel.energy.consumption..L.hr., breaks = $\mathrm{c}(4,4.2,4.4,4.6,4.8,5,5.2,5.4,5.6,5.8,6,6.2,6.4,6.6)$,

col="red", main = "System OFF", xlab= "Fossil fuel energy consumption $(\mathrm{MJ} / \mathrm{h}) ")$

hist(SystemONData $\$$ Average.Fossil.Fuel.energy.consumption..L.hr., breaks= c(4.0,4.2,4.4,4.6,4.8,5,5.2,5.4,5.6,5.8,6,6.2,6.4,6.6),

$\mathrm{col}=$ "green", main = "System ON", xlab= "Fossil fuel energy consumption $(\mathrm{MJ} / \mathrm{h}) ")$

hist(SystemOFFData\$idiling, breaks = 20, col="red", main = "System OFF", xlab= "Fraction of time spent idiling")

hist(SystemONData\$idiling, breaks= 20, col="green", main = "System ON", xlab= "Fraction of time spent idiling")

hist(SystemOFFData\$idiling, breaks = 10, col="red", main = "System OFF", xlab= "Fraction of time spent idiling" )

hist(SystemONData\$idiling, breaks=10, col="green", main = "System ON", xlab= "Fraction of time spent idiling")

hist(SystemOFFData\$braking, breaks = 20, col="red", main = "System OFF", xlab= "Fraction of time spent braking")

hist(SystemONData\$braking, breaks= 20, col="green", main = "System ON", xlab= "Fraction of time spent braking")

hist(SystemOFFData\$braking, breaks = 10, col="red", main = "System OFF", xlab= "Fraction of time spent braking")

hist(SystemONData\$braking, breaks= 10, col="green", main = "System ON", xlab= "Fraction of time spent braking")

hist(SystemOFFData\$S50, col="red", main = "System OFF", breaks = c(1,2,3,4,5,6,7), xlab= "Number of stops")

hist(SystemONData\$S50, col="green", main = "System ON", breaks = c(1,2,3,4,5,6,7), xlab= "Number of stops")

hist(SystemOFFData\$S150, col="red", main = "System OFF", xlab= "Number of stops")

hist(SystemONData\$S150, col="green", main = "System ON", xlab= "Number of stops")

ggplot $($ DataToSigTestP, aes $($ S50, colour $=$ Prefix $))+$ geom_freqpoly $($ na.rm $=$ FALSE, show.legend $=$ NA, inherit.aes $=$ TRUE, bins=4)+ ggtitle("Stops") +

xlab("Number of stops")

ggplot(DataToSigTestP, aes(idiling, colour $=$ Prefix, $x l a b=$ "Number of stops") $)+$ geom_freqpoly $($ na.rm $=$ FALSE, show.legend $=$ NA,

inherit.aes $=$ TRUE, bins=6) + ggtitle("idiling") + xlab( "Fraction of time idiling")

ggplot(DataToSigTestP, aes(braking, colour $=$ Prefix $))+$ geom_freqpoly $($ na.rm $=$ FALSE,

show.legend $=$ NA, inherit.aes $=$ TRUE, bins=6) + ggtitle("braking") $+x l a b($ "Fraction of time braking")

ggplot(DataToSigTestP, aes(Average.Fossil.Fuel.energy.consumption..L.hr., colour $=$ Prefix $)$ ) +

geom_freqpoly ( na.rm = FALSE,show.legend = NA, inherit.aes = TRUE, bins=6) + ggtitle("Fuel") + xlab("Fuel consumption $\left.(\mathrm{L} / \mathrm{h})^{\prime \prime}\right)$

ggplot(DataToSigTestP, aes(CO2.Equivalent.Produced..Kg.hr., colour = Prefix $))^{+}$

geom_freqpoly ( na.rm = FALSE,show.legend $=$ NA, inherit.aes $=$ TRUE, bins $=6)+$ ggtitle("CO2E” $)+$ xlab(“CO2 equivaleng emissions $(\mathrm{kg} / \mathrm{h})$ ")

ggplot(DataToSigTestP, aes(Average.Trip.Speed..Km.h., colour $=$ Prefix $))+$

geom_freqpoly $($ na.rm $=$ FALSE,show.legend $=$ NA, inherit.aes $=$ TRUE, bins=6) + ggtitle("Average Speed") + xlab(“Average Speed (km/h)") 
Additional confidence interval analysis

library(wBoot)

\#separating out key variables

ONSpeed<- SystemONAll\$Average.Trip.Speed..Km.h.

OFFSpeed<- SystemOFFAll\$Average.Trip.Speed..Km.h.

ONFuel<- SystemONAll\$Average.Fossil.Fuel.energy.consumption..L.hr.

OFFFuel<- SystemOFFAll\$Average.Fossil.Fuel.energy.consumption..L.hr.

ONCO2E $<-$ SystemONAll\$CO2.Equivalent.Produced..Kg.hr.

OFFCO2E<- SystemOFFAll\$CO2.Equivalent.Produced..Kg.hr.

\#bootstrapping to create $95 \%$ confidence intervals

boot.paired.bca $(\mathrm{ONCO} 2 \mathrm{E}, \mathrm{OFFCO} 2 \mathrm{E}$, variable $=\mathrm{NULL}$, null.hyp $=$ NULL,

alternative $=\mathrm{c}$ ("two.sided"),

conf.level $=0.95$, type $=$ NULL, $R=9999)$

boot.paired.bca(ONFuel, OFFFuel, variable $=$ NULL, null.hyp $=$ NULL,

alternative $=\mathrm{c}$ (“two.sided"),

conf.level $=0.95$, type $=$ NULL, $R=9999)$

boot.paired.bca(ONSpeed, OFFSpeed, variable $=$ NULL, null.hyp $=$ NULL,

alternative $=\mathrm{c}$ ("two.sided"),

conf.level $=0.95$, type $=$ NULL, $R=9999$ ) 


\section{Appendix F R Outputs}

\begin{tabular}{|c|c|c|c|c|c|}
\hline$\Delta$ & Average.Trip.Speed..Km.h. & CO2.Equivalent.Produced..Kg.hr. & Average.Fossil.Fuel.energy.consumption..L.hr. & idiling $₹$ & breaking $\neq$ \\
\hline nobs & 50.000000 & 50.000000 & 50.000000 & 50.000000 & 50.000000 \\
\hline NAs & 0.000000 & 0.000000 & 0.000000 & 0.000000 & 0.000000 \\
\hline Minimum & 32.530000 & 11.990000 & 4.470000 & 0.050109 & 0.034274 \\
\hline Maximum & 52.170000 & 17.240000 & 6.430000 & 0.336585 & 0.152921 \\
\hline 1. Quartile & 37.855000 & 13.080000 & 4.877500 & 0.147199 & 0.067365 \\
\hline 3. Quartile & 44.152500 & 14.447500 & 5.390000 & 0.237281 & 0.095016 \\
\hline Mean & 41.254200 & 13.939600 & 5.199400 & 0.192629 & 0.082740 \\
\hline Median & 40.755000 & 14.070000 & 5.245000 & 0.195227 & 0.087165 \\
\hline Sum & 2062.710000 & 696.980000 & 259.970000 & 9.631436 & 4.136997 \\
\hline SE Mean & 0.665600 & 0.158944 & 0.059397 & 0.009167 & 0.003108 \\
\hline LCL Mean & 39.916626 & 13.620191 & 5.080038 & 0.174206 & 0.076494 \\
\hline UCL Mean & 42.591774 & 14.259009 & 5.318762 & 0.211051 & 0.088986 \\
\hline Variance & 22.151184 & 1.263155 & 0.176398 & 0.004202 & 0.000483 \\
\hline Stdev & 4.706504 & 1.123902 & 0.419997 & 0.064824 & 0.021976 \\
\hline Skewness & 0.246735 & 0.501324 & 0.498644 & -0.035865 & 0.257226 \\
\hline Kurtosis & -0.570468 & 0.293156 & 0.278372 & -0.508545 & 0.712090 \\
\hline
\end{tabular}

Figure 33: All recorded System OFF summary statistics

\begin{tabular}{|c|c|c|c|c|c|}
\hline$\Delta$ & Average.Trip.Speed..Km.h. & CO2.Equivalent.Produced..Kg.hr. & Average.Fossil.Fuel.energy.consumption..L.hr. & idiling $\hat{\vee}$ & breaking $\neq$ \\
\hline nobs & 70.000000 & 70.000000 & 70.000000 & 70.000000 & 70.000000 \\
\hline NAs & 0.000000 & 0.000000 & 0.000000 & 0.000000 & 0.000000 \\
\hline Minimum & 14.420000 & 8.700000 & 3.240000 & 0.000000 & 0.000000 \\
\hline Maximum & 48.570000 & 14.490000 & 5.410000 & 0.426897 & 0.227957 \\
\hline 1. Quartile & 36.230000 & 11.845000 & 4.420000 & 0.012903 & 0.007000 \\
\hline 3. Quartile & 41.145000 & 13.100000 & 4.895000 & 0.255416 & 0.069504 \\
\hline Mean & 37.554857 & 12.341286 & 4.604143 & 0.166123 & 0.048406 \\
\hline Median & 38.740000 & 12.405000 & 4.625000 & 0.188875 & 0.050331 \\
\hline Sum & 2628.840000 & 863.890000 & 322.290000 & 11.628626 & 3.388419 \\
\hline SE Mean & 0.789010 & 0.129123 & 0.048360 & 0.015013 & 0.004679 \\
\hline LCL Mean & 35.980824 & 12.083692 & 4.507667 & 0.136173 & 0.039072 \\
\hline UCL Mean & 39.128890 & 12.598880 & 4.700619 & 0.196073 & 0.057740 \\
\hline Variance & 43.577625 & 1.167098 & 0.163709 & 0.015777 & 0.001532 \\
\hline Stdev & 6.601335 & 1.080323 & 0.404609 & 0.125608 & 0.039146 \\
\hline Skewness & -1.064893 & -0.677778 & -0.679736 & 0.055577 & 1.270566 \\
\hline Kurtosis & 1.250415 & 0.789462 & 0.780425 & -1.093963 & 4.589361 \\
\hline
\end{tabular}

Figure 34: All recorded IVU active summary statistics 


\begin{tabular}{|c|c|c|c|c|c|}
\hline$\Delta$ & Average.Trip.Speed..Km.h. & CO2.Equivalent.Produced..Kg.hr. & Average.Fossil.Fuel.energy.consumption..L.hr. & idiling $₹$ & breaking $₹$ \\
\hline nobs & 18.000000 & 18.000000 & 18.000000 & 18 & 18 \\
\hline NAs & 0.000000 & 0.000000 & 0.000000 & 0 & 0 \\
\hline Minimum & 31.300000 & 11.380000 & 4.250000 & 0 & 0 \\
\hline Maximum & 46.800000 & 14.490000 & 5.410000 & 0 & 0 \\
\hline 1. Quartile & 37.925000 & 12.105000 & 4.522500 & 0 & 0 \\
\hline 3. Quartile & 43.375000 & 13.350000 & 4.987500 & 0 & 0 \\
\hline Mean & 40.083333 & 12.798889 & 4.781667 & 0 & 0 \\
\hline Median & 40.000000 & 12.950000 & 4.840000 & 0 & 0 \\
\hline Sum & 721.500000 & 230.380000 & 86.070000 & 0 & 0 \\
\hline SE Mean & 1.014511 & 0.193291 & 0.072411 & 0 & 0 \\
\hline LCL Mean & 37.942903 & 12.391081 & 4.628894 & 0 & 0 \\
\hline UCL Mean & 42.223764 & 13.206697 & 4.934440 & 0 & 0 \\
\hline Variance & 18.526176 & 0.672505 & 0.094379 & 0 & 0 \\
\hline Stdev & 4.304205 & 0.820064 & 0.307212 & 0 & 0 \\
\hline Skewness & -0.430124 & -0.040502 & -0.051303 & $\mathrm{NaN}$ & $\mathrm{NaN}$ \\
\hline Kurtosis & -0.649518 & -0.792774 & -0.810395 & $\mathrm{NaN}$ & $\mathrm{NaN}$ \\
\hline
\end{tabular}

Figure 35: All recorded IVU providing countdowns only summary statistics

\begin{tabular}{|c|c|c|c|c|c|}
\hline $\boldsymbol{\Delta}$ & Average.Trip.Speed..Km.h. & CO2.Equivalent.Produced..Kg.hr. & Average.Fossil.Fuel.energy.consumption...L.hr. & idiling $\hat{*}$ & breaking \\
\hline nobs & 52.000000 & 52.000000 & 52.000000 & 52.000000 & 52.000000 \\
\hline NAs & 0.000000 & 0.000000 & 0.000000 & 0.000000 & 0.000000 \\
\hline Minimum & 14.420000 & 8.700000 & 3.240000 & 0.051613 & 0.028000 \\
\hline Maximum & 48.570000 & 14.490000 & 5.400000 & 0.426897 & 0.227957 \\
\hline 1. Quartile & 32.807500 & 11.650000 & 4.340000 & 0.150983 & 0.048368 \\
\hline 3. Quartile & 40.622500 & 12.755000 & 4.760000 & 0.277481 & 0.074955 \\
\hline Mean & 36.679615 & 12.182885 & 4.542692 & 0.223627 & 0.065162 \\
\hline Median & 37.875000 & 12.325000 & 4.595000 & 0.223582 & 0.060686 \\
\hline Sum & 1907.340000 & 633.510000 & 236.220000 & 11.628626 & 3.388419 \\
\hline SE Mean & 0.978078 & 0.155375 & 0.058021 & 0.012584 & 0.004297 \\
\hline LCL Mean & 34.716042 & 11.870956 & 4.426210 & 0.198365 & 0.056536 \\
\hline UCL Mean & 38.643189 & 12.494814 & 4.659175 & 0.248890 & 0.073788 \\
\hline Variance & 49.745082 & 1.255358 & 0.175055 & 0.008234 & 0.000960 \\
\hline Stdev & 7.053019 & 1.120428 & 0.418396 & 0.090742 & 0.030983 \\
\hline Skewness & -0.921378 & -0.631758 & -0.635979 & 0.203282 & 3.020169 \\
\hline Kurtosis & 0.678755 & 0.538645 & 0.539677 & -0.507870 & 12.630696 \\
\hline
\end{tabular}

Figure 36: All recorded IVU providing EcoDrive recommendation summary statistics

\section{7}




\begin{tabular}{|c|c|c|c|c|c|}
\hline $\boldsymbol{\Delta}$ & Average.Trip.Speed..Km.h. & CO2.Equivalent.Produced...Kg.hr. & Average.Fossil.Fuel.energy.consumption..L.hr. & idiling $\hat{\vee}$ & breaking \\
\hline nobs & 50.000000 & 50.000000 & 50.000000 & 50.000000 & 50.000000 \\
\hline NAs & 0.000000 & 0.000000 & 0.000000 & 0.000000 & 0.000000 \\
\hline Minimum & 14.420000 & 8.700000 & 3.240000 & 0.051613 & 0.028000 \\
\hline Maximum & 48.570000 & 14.490000 & 5.400000 & 0.426897 & 0.227957 \\
\hline 1. Quartile & 33.260000 & 11.702500 & 4.360000 & 0.150267 & 0.048329 \\
\hline 3. Quartile & 40.747500 & 12.750000 & 4.760000 & 0.276957 & 0.074957 \\
\hline Mean & 36.849600 & 12.169400 & 4.537600 & 0.223955 & 0.065297 \\
\hline Median & 38.035000 & 12.325000 & 4.595000 & 0.223582 & 0.060686 \\
\hline Sum & 1842.480000 & 608.470000 & 226.880000 & 11.197748 & 3.264841 \\
\hline SE Mean & 1.001072 & 0.157288 & 0.058736 & 0.012972 & 0.004463 \\
\hline LCL Mean & 34.837870 & 11.853318 & 4.419565 & 0.197886 & 0.056328 \\
\hline UCL Mean & 38.861330 & 12.485482 & 4.655635 & 0.250023 & 0.074265 \\
\hline Variance & 50.107265 & 1.236973 & 0.172496 & 0.008414 & 0.000996 \\
\hline Stdev & 7.078649 & 1.112193 & 0.415327 & 0.091727 & 0.031558 \\
\hline Skewness & -0.978062 & -0.683881 & -0.688399 & 0.198993 & 2.960977 \\
\hline Kurtosis & 0.810250 & 0.661842 & 0.661832 & -0.529506 & 12.053539 \\
\hline
\end{tabular}

Figure 37: All informed driver runs without replacement

\begin{tabular}{|c|c|c|c|c|c|}
\hline $\boldsymbol{\Delta}$ & Average.Trip.Speed..Km.h. & CO2.Equivalent.Produced...Kg.hr. & Average.Fossil.Fuel.energy.consumption..L.hr. & idiling $₹$ & breaking \\
\hline nobs & 46.000000 & 46.000000 & 46.000000 & 46.000000 & 46.000000 \\
\hline NAs & 0.000000 & 0.000000 & 0.000000 & 0.000000 & 0.000000 \\
\hline Minimum & 33.825355 & 11.992414 & 4.472039 & 0.050109 & 0.034274 \\
\hline Maximum & 52.171617 & 17.244839 & 6.434390 & 0.336585 & 0.152921 \\
\hline 1. Quartile & 37.855844 & 13.082431 & 4.879288 & 0.147199 & 0.067100 \\
\hline 3. Quartile & 44.726058 & 14.444578 & 5.388177 & 0.237281 & 0.097259 \\
\hline Mean & 41.436636 & 13.942644 & 5.200652 & 0.192153 & 0.082420 \\
\hline Median & 40.752650 & 14.020245 & 5.229634 & 0.196198 & 0.086180 \\
\hline Sum & 1906.085270 & 641.361642 & 239.229981 & 8.839025 & 3.791299 \\
\hline SE Mean & 0.697099 & 0.169095 & 0.063175 & 0.009584 & 0.003362 \\
\hline LCL Mean & 40.032607 & 13.602069 & 5.073410 & 0.172850 & 0.075647 \\
\hline UCL Mean & 42.840665 & 14.283219 & 5.327893 & 0.211455 & 0.089192 \\
\hline Variance & 22.353542 & 1.315285 & 0.183591 & 0.004225 & 0.000520 \\
\hline Stdev & 4.727953 & 1.146859 & 0.428476 & 0.065000 & 0.022805 \\
\hline Skewness & 0.287669 & 0.545081 & 0.545157 & -0.110829 & 0.289175 \\
\hline Kurtosis & -0.712145 & 0.252359 & 0.252627 & -0.517507 & 0.492802 \\
\hline
\end{tabular}

Figure 38: Uninformed driver final summary a

\section{8}




\begin{tabular}{|c|c|c|c|c|c|c|c|c|c|c|}
\hline S20 & S50 & S100 & S150 & AveGrade & LowSpeedCoasting..under25MPH. & X0.3 & X3to6 $=$ & X6to9 $=$ & X9to12 & above12 \\
\hline 46.000000 & 46.000000 & 46.000000 & 46.000000 & 46.000000 & 46.000000 & 46.000000 & 46.000000 & 46.000000 & 46.000000 & 46.000000 \\
\hline 0.000000 & 0.000000 & 0.000000 & 0.000000 & 0.000000 & 0.000000 & 0.000000 & 0.000000 & 0.000000 & 0.000000 & 0.000000 \\
\hline 2.000000 & 2.000000 & 2.000000 & 2.000000 & -0.290000 & 0.013363 & 0.008658 & 0.004098 & 0.006342 & 0.008197 & 0.000000 \\
\hline 7.000000 & 7.000000 & 7.000000 & 7.000000 & 0.250000 & 0.097913 & 0.100977 & 0.039936 & 0.060606 & 0.044776 & 0.046185 \\
\hline 3.000000 & 3.000000 & 3.000000 & 3.000000 & -0.180000 & 0.030024 & 0.020319 & 0.012033 & 0.021558 & 0.016915 & 0.006996 \\
\hline 5.000000 & 5.000000 & 5.000000 & 5.000000 & 0.180000 & 0.047342 & 0.034399 & 0.022959 & 0.038067 & 0.033290 & 0.028620 \\
\hline 4.173913 & 4.152174 & 4.130435 & 4.021739 & -0.005217 & 0.041389 & 0.031038 & 0.018214 & 0.029319 & 0.023768 & 0.018552 \\
\hline 4.000000 & 4.000000 & 4.000000 & 4.000000 & -0.125000 & 0.038172 & 0.026252 & 0.017423 & 0.028073 & 0.020772 & 0.017934 \\
\hline 192.000000 & 191.000000 & 190.000000 & 185.000000 & -0.240000 & 1.903901 & 1.427765 & 0.837840 & 1.348693 & 1.093349 & 0.853398 \\
\hline 0.204548 & 0.198952 & 0.200503 & 0.190319 & 0.027904 & 0.002712 & 0.002621 & 0.001385 & 0.001991 & 0.001345 & 0.001910 \\
\hline 3.761932 & 3.751463 & 3.726600 & 3.638417 & -0.061419 & 0.035926 & 0.025759 & 0.015424 & 0.025309 & 0.021059 & 0.014706 \\
\hline 4.585894 & 4.552884 & 4.534269 & 4.405062 & 0.050984 & 0.046852 & 0.036317 & 0.021004 & 0.033330 & 0.026478 & 0.022399 \\
\hline 1.924638 & 1.820773 & 1.849275 & 1.666184 & 0.035817 & 0.000338 & 0.000316 & 0.000088 & 0.000182 & 0.000083 & 0.000168 \\
\hline 1.387313 & 1.349360 & 1.359881 & 1.290807 & 0.189253 & 0.018395 & 0.017777 & 0.009394 & 0.013504 & 0.009124 & 0.012953 \\
\hline 0.428020 & 0.366610 & 0.391895 & 0.445853 & 0.055720 & 1.066253 & 1.837867 & 0.808004 & 0.275366 & 0.456679 & 0.167992 \\
\hline-0.607497 & -0.650023 & -0.687145 & -0.565053 & -1.906180 & 0.845738 & 4.019370 & -0.072630 & -0.749641 & -1.002956 & -1.368238 \\
\hline
\end{tabular}

Figure 39: Uninformed driver final summary b

\begin{tabular}{|c|c|c|c|c|c|c|c|c|c|}
\hline ModSpeedCoasting25to50MPH & X0to3 & X3to6.1 & X6to9.1 & X9to12.1 & above12.1 & X12to18 & X18to24 & $\mathrm{X} 24$ to 30 & over30 \\
\hline 46.000000 & 46.000000 & 46.000000 & 46.000000 & 46.000000 & 46.000000 & 46.000000 & 46.000000 & 46.000000 & 46.000000 \\
\hline 0.000000 & 0.000000 & 0.000000 & 0.000000 & 0.000000 & 0.000000 & 0.000000 & 0.000000 & 0.000000 & 0.000000 \\
\hline 0.050691 & 0.042435 & 0.035593 & 0.035635 & 0.035941 & 0.000000 & 0.004815 & 0.000000 & 0.000000 & 0.000000 \\
\hline 0.174877 & 0.171975 & 0.151210 & 0.155172 & 0.125581 & 0.046185 & 0.159091 & 0.046512 & 0.009756 & 0.010060 \\
\hline 0.090379 & 0.073630 & 0.063130 & 0.067796 & 0.069353 & 0.006996 & 0.048003 & 0.001872 & 0.000000 & 0.000000 \\
\hline 0.127748 & 0.114937 & 0.110883 & 0.101845 & 0.088606 & 0.028620 & 0.088069 & 0.019801 & 0.001913 & 0.000000 \\
\hline 0.111099 & 0.095943 & 0.087528 & 0.086161 & 0.081045 & 0.018552 & 0.071050 & 0.012501 & 0.001491 & 0.000769 \\
\hline 0.109249 & 0.100155 & 0.084864 & 0.088431 & 0.078588 & 0.017934 & 0.069900 & 0.009435 & 0.000000 & 0.000000 \\
\hline 5.110572 & 4.413389 & 4.026302 & 3.963428 & 3.728054 & 0.853398 & 3.268303 & 0.575059 & 0.068601 & 0.035397 \\
\hline 0.004465 & 0.004442 & 0.004456 & 0.003909 & 0.002927 & 0.001910 & 0.004460 & 0.001949 & 0.000399 & 0.000300 \\
\hline 0.102107 & 0.086998 & 0.078553 & 0.078288 & 0.075150 & 0.014706 & 0.062068 & 0.008576 & 0.000688 & 0.000166 \\
\hline 0.120092 & 0.104889 & 0.096504 & 0.094035 & 0.086940 & 0.022399 & 0.080032 & 0.016427 & 0.002295 & 0.001373 \\
\hline 0.000917 & 0.000907 & 0.000913 & 0.000703 & 0.000394 & 0.000168 & 0.000915 & 0.000175 & 0.000007 & 0.000004 \\
\hline 0.030282 & 0.030124 & 0.030224 & 0.026513 & 0.019851 & 0.012953 & 0.030246 & 0.013218 & 0.002706 & 0.002034 \\
\hline 0.144563 & 0.224632 & 0.218961 & 0.366734 & 0.267393 & 0.167992 & 0.362064 & 0.961723 & 1.862734 & 3.222620 \\
\hline-0.792837 & -0.463059 & -0.891450 & -0.223019 & -0.252127 & -1.368238 & 0.329259 & -0.184392 & 2.256282 & 10.547074 \\
\hline
\end{tabular}

Figure 40: Uninformed driver final summary $c$ 


\begin{tabular}{|r|r|r|r|r|r|}
\hline over50MPH..6 & X6to12 & X12to18.1 & X18to24.1 & X24to30.1 & \multicolumn{1}{l|}{ above30 } \\
\hline 46.000000 & 46.000000 & 46.000000 & 46.000000 & 46.000000 & 46.000000 \\
\hline 0.000000 & 0.000000 & 0.000000 & 0.000000 & 0.000000 & 0.000000 \\
\hline 0.000000 & 0.000000 & 0.000000 & 0.000000 & 0.000000 & 0.000000 \\
\hline 0.038055 & 0.050740 & 0.022272 & 0.004454 & 0.002012 & 0.001626 \\
\hline 0.000000 & 0.000000 & 0.000000 & 0.000000 & 0.000000 & 0.000000 \\
\hline 0.011581 & 0.006594 & 0.002991 & 0.000000 & 0.000000 & 0.000000 \\
\hline 0.007218 & 0.005629 & 0.002400 & 0.000231 & 0.000044 & 0.000035 \\
\hline 0.332006 & 0.258954 & 0.110408 & 0.010620 & 0.002012 & 0.001626 \\
\hline 0.004493 & 0.002746 & 0.000925 & -0.000012 & -0.000044 & -0.000036 \\
\hline 0.009943 & 0.008513 & 0.003875 & 0.000474 & 0.000132 & 0.000107 \\
\hline 0.000084 & 0.000094 & 0.000025 & 0.000001 & 0.000000 & 0.000000 \\
\hline 0.009176 & 0.009711 & 0.004966 & 0.000819 & 0.000297 & 0.000240 \\
\hline 1.458711 & 2.665993 & 2.376326 & 3.708634 & 6.346415 & 6.346415 \\
\hline 1.716360 & 8.308443 & 5.472452 & 14.112787 & 39.129017 & 39.129017 \\
\hline
\end{tabular}

Figure 41: Uninformed driver final summary d

\begin{tabular}{|c|c|c|c|c|c|}
\hline $\boldsymbol{\Delta}$ & Average.Trip.Speed..Km.h. & CO2.Equivalent.Produced..Kg.hr. & Average.Fossil.Fuel.energy.consumption..L.hr. & idiling $=$ & breaking \\
\hline nobs & 38.000000 & 38.000000 & 38.000000 & 38.000000 & 38.000000 \\
\hline NAS & 0.000000 & 0.000000 & 0.000000 & 0.000000 & 0.000000 \\
\hline Minimum & 30.284569 & 10.942148 & 4.079616 & 0.051613 & 0.028000 \\
\hline Maximum & 48.573845 & 14.485593 & 5.403507 & 0.339943 & 0.227957 \\
\hline 1. Quartile & 37.513784 & 12.158482 & 4.534012 & 0.141633 & 0.047748 \\
\hline 3. Quartile & 42.038006 & 12.975841 & 4.839409 & 0.247329 & 0.076250 \\
\hline Mean & 39.705076 & 12.600869 & 4.699330 & 0.193586 & 0.068135 \\
\hline Median & 39.542294 & 12.497749 & 4.660791 & 0.209240 & 0.061721 \\
\hline Sum & 1508.792893 & 478.833029 & 178.574545 & 7.356263 & 2.589149 \\
\hline SE Mean & 0.681390 & 0.116838 & 0.043653 & 0.012066 & 0.005728 \\
\hline LCL Mean & 38.324448 & 12.364133 & 4.610880 & 0.169138 & 0.056529 \\
\hline UCL Mean & 41.085704 & 12.837606 & 4.787780 & 0.218034 & 0.079742 \\
\hline Variance & 17.643123 & 0.518743 & 0.072413 & 0.005532 & 0.001247 \\
\hline Stdev & 4.200372 & 0.720238 & 0.269097 & 0.074379 & 0.035311 \\
\hline Skewness & -0.087595 & 0.381134 & 0.381162 & -0.074829 & 2.570203 \\
\hline Kurtosis & -0.224132 & 0.076837 & 0.076796 & -0.925838 & 8.755653 \\
\hline
\end{tabular}

Figure 42: Informed driver final summary a 


\begin{tabular}{|c|c|c|c|c|c|c|c|c|c|c|}
\hline S20 & S50 & S100 & S150 & AveGrade & LowSpeedCoasting..under25MPH. & $\mathrm{x} 0.3$ & X3to6 $\hat{\vee}$ & X6to9 $=$ & X9to12 $=$ & above12 \\
\hline 157.000000 & 157.000000 & 156.000000 & 152.000000 & -0.330000 & 2.067477 & 1.431750 & 0.645236 & 1.009856 & 1.000842 & 0.594949 \\
\hline 38.000000 & 38.000000 & 38.000000 & 38.000000 & 38.000000 & 38.000000 & 38.000000 & 38.000000 & 38.000000 & 38.000000 & 38.000000 \\
\hline 7.000000 & 7.000000 & 7.000000 & 6.000000 & 0.230000 & 0.149515 & 0.104530 & 0.063985 & 0.056673 & 0.050467 & 0.042553 \\
\hline 5.000000 & 5.000000 & 5.000000 & 5.000000 & 0.170000 & 0.068547 & 0.047070 & 0.020663 & 0.032244 & 0.032123 & 0.021103 \\
\hline 4.603718 & 4.603718 & 4.568779 & 4.452103 & 0.051300 & 0.064191 & 0.044860 & 0.020628 & 0.030018 & 0.029769 & 0.019304 \\
\hline 4.131579 & 4.131579 & 4.105263 & 4.000000 & -0.008684 & 0.054407 & 0.037678 & 0.016980 & 0.026575 & 0.026338 & 0.015657 \\
\hline 4.000000 & 4.000000 & 4.000000 & 4.000000 & -0.100000 & 0.052073 & 0.035619 & 0.014016 & 0.024772 & 0.024718 & 0.015575 \\
\hline 3.659440 & 3.659440 & 3.641747 & 3.547897 & -0.068668 & 0.044623 & 0.030495 & 0.013332 & 0.023132 & 0.022907 & 0.012009 \\
\hline 3.000000 & 3.000000 & 3.000000 & 3.000000 & -0.177500 & 0.033819 & 0.022593 & 0.010068 & 0.020532 & 0.018483 & 0.007354 \\
\hline 2.063300 & 2.063300 & 1.988620 & 1.891892 & 0.033304 & 0.000886 & 0.000477 & 0.000123 & 0.000110 & 0.000109 & 0.000123 \\
\hline 1.436419 & 1.436419 & 1.410184 & 1.375461 & 0.182493 & 0.029766 & 0.021851 & 0.011098 & 0.010474 & 0.010438 & 0.011096 \\
\hline 1.000000 & 1.000000 & 1.000000 & 1.000000 & -0.330000 & 0.009141 & 0.004000 & 0.002198 & 0.006000 & 0.010791 & 0.000000 \\
\hline 0.233018 & 0.233018 & 0.228762 & 0.223129 & 0.029604 & 0.004829 & 0.003545 & 0.001800 & 0.001699 & 0.001693 & 0.001800 \\
\hline 0.000000 & 0.000000 & 0.000000 & 0.000000 & 0.000000 & 0.000000 & 0.000000 & 0.000000 & 0.000000 & 0.000000 & 0.000000 \\
\hline-0.117397 & -0.117397 & -0.124618 & -0.182031 & 0.005433 & 0.874696 & 1.005709 & 2.103428 & 0.725362 & 0.510960 & 0.454308 \\
\hline-0.898196 & -0.898196 & -0.826021 & -0.985467 & -1.813506 & 1.067956 & 1.174169 & 6.189004 & 0.385734 & -0.592719 & -0.621609 \\
\hline
\end{tabular}

Figure 43: Informed driver final summary $b$

\begin{tabular}{|c|c|c|c|c|c|c|c|c|c|}
\hline ModSpeedCoasting25to50MPH & X0to3 & X3to6.1 & X6to9.1 & X9to12.1 & above12.1 & $\mathrm{X} 12$ to18 & X18to24 & X24to30 & over30 \\
\hline 5.127189 & 4.113870 & 3.330428 & 2.942630 & 2.689881 & 0.594949 & 2.356139 & 0.342178 & 0.033174 & 0.039265 \\
\hline 38.000000 & 38.000000 & 38.000000 & 38.000000 & 38.000000 & 38.000000 & 38.000000 & 38.000000 & 38.000000 & 38.000000 \\
\hline 0.213884 & 0.204396 & 0.142857 & 0.147368 & 0.115174 & 0.042553 & 0.114416 & 0.041841 & 0.005666 & 0.010753 \\
\hline 0.149974 & 0.136036 & 0.100232 & 0.092156 & 0.083154 & 0.021103 & 0.079298 & 0.015377 & 0.001811 & 0.000000 \\
\hline 0.144942 & 0.119316 & 0.095639 & 0.086442 & 0.077308 & 0.019304 & 0.069972 & 0.012049 & 0.001330 & 0.001769 \\
\hline 0.134926 & 0.108260 & 0.087643 & 0.077438 & 0.070786 & 0.015657 & 0.062004 & 0.009005 & 0.000873 & 0.001033 \\
\hline 0.136153 & 0.099346 & 0.085742 & 0.071967 & 0.068072 & 0.015575 & 0.063420 & 0.006626 & 0.000000 & 0.000000 \\
\hline 0.124910 & 0.097203 & 0.079646 & 0.068433 & 0.064265 & 0.012009 & 0.054036 & 0.005961 & 0.000416 & 0.000297 \\
\hline 0.114235 & 0.086488 & 0.073221 & 0.065040 & 0.059526 & 0.007354 & 0.045289 & 0.001855 & 0.000000 & 0.000000 \\
\hline 0.000929 & 0.001132 & 0.000592 & 0.000751 & 0.000394 & 0.000123 & 0.000588 & 0.000086 & 0.000002 & 0.000005 \\
\hline 0.030472 & 0.033639 & 0.024329 & 0.027396 & 0.019841 & 0.011096 & 0.024242 & 0.009261 & 0.001390 & 0.002239 \\
\hline 0.058577 & 0.046763 & 0.041076 & 0.031161 & 0.024079 & 0.000000 & 0.012632 & 0.000000 & 0.000000 & 0.000000 \\
\hline 0.004943 & 0.005457 & 0.003947 & 0.004444 & 0.003219 & 0.001800 & 0.003933 & 0.001502 & 0.000225 & 0.000363 \\
\hline 0.000000 & 0.000000 & 0.000000 & 0.000000 & 0.000000 & 0.000000 & 0.000000 & 0.000000 & 0.000000 & 0.000000 \\
\hline 0.287296 & 0.574697 & 0.190527 & 0.593127 & 0.154462 & 0.454308 & -0.007166 & 1.372298 & 1.511957 & 2.527371 \\
\hline 0.767141 & 0.016766 & -0.363046 & -0.174377 & 0.108271 & -0.621609 & -0.665319 & 2.070777 & 1.825268 & 7.046540 \\
\hline
\end{tabular}

Figure 44: Informed driver final summary c 


\begin{tabular}{|r|r|r|r|r|r|}
\hline over50MPH..6 & X6to12 & X12to18.1 & X18to24.1 & X24to30.1 & \multicolumn{1}{l|}{ above30 } \\
\hline 0.145047 & 0.103678 & 0.069351 & 0.004925 & 0.001799 & 0.004925 \\
\hline 38.000000 & 38.000000 & 38.000000 & 38.000000 & 38.000000 & 38.000000 \\
\hline 0.026119 & 0.025105 & 0.017986 & 0.002833 & 0.001799 & 0.002833 \\
\hline 0.004880 & 0.002674 & 0.001765 & 0.000000 & 0.000000 & 0.000000 \\
\hline 0.006078 & 0.004680 & 0.003222 & 0.000315 & 0.000143 & 0.000315 \\
\hline 0.003817 & 0.002728 & 0.001825 & 0.000130 & 0.000047 & 0.000130 \\
\hline 0.000000 & 0.000000 & 0.000000 & 0.000000 & 0.000000 & 0.000000 \\
\hline 0.000000 & 0.000000 & 0.000000 & 0.000000 & 0.000000 & 0.000000 \\
\hline 0.006879 & 0.005939 & 0.004249 & 0.000564 & 0.000292 & 0.000564 \\
\hline 0.000000 & 0.000000 & 0.000000 & 0.000000 & 0.000000 & 0.000000 \\
\hline 0.001116 & 0.000963 & 0.000689 & 0.000091 & 0.000047 & 0.000091 \\
\hline 0.000000 & 0.000000 & 0.000000 & 0.000000 & 0.000000 & 0.000000 \\
\hline 1.710768 & 2.791750 & 2.733179 & 3.997808 & 5.686288 & 3.997808 \\
\hline 1.840755 & 7.269284 & 6.851397 & 14.766638 & 31.155817 & 14.766638 \\
\hline & 0.000035 & 0.000018 & 0.000000 & 0.000000 & 0.000000 \\
\hline
\end{tabular}

Figure 45: Informed driver final summaryd 


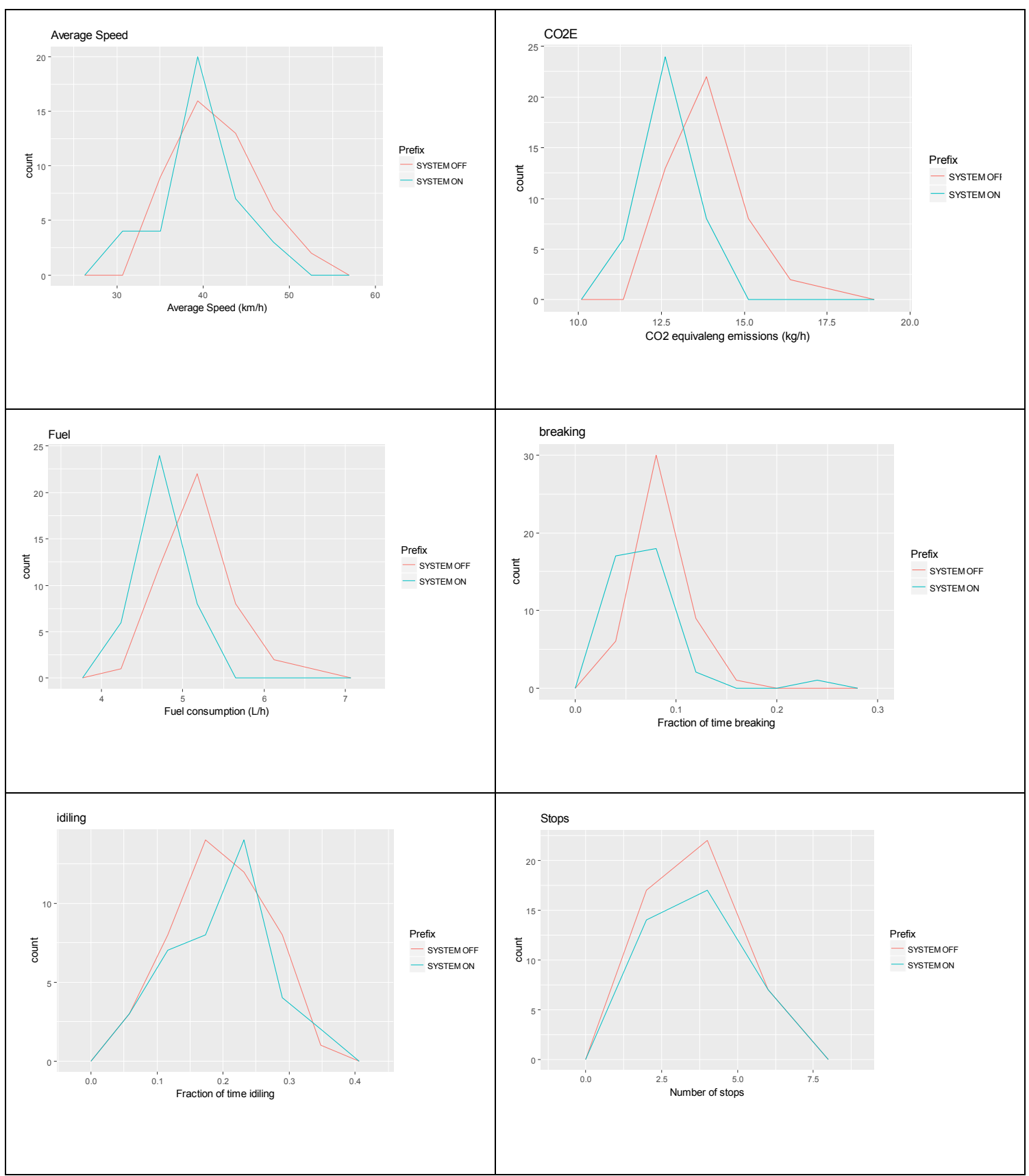

Figure 46: Frequency distributions of variables for uninformed and informed driver phases 


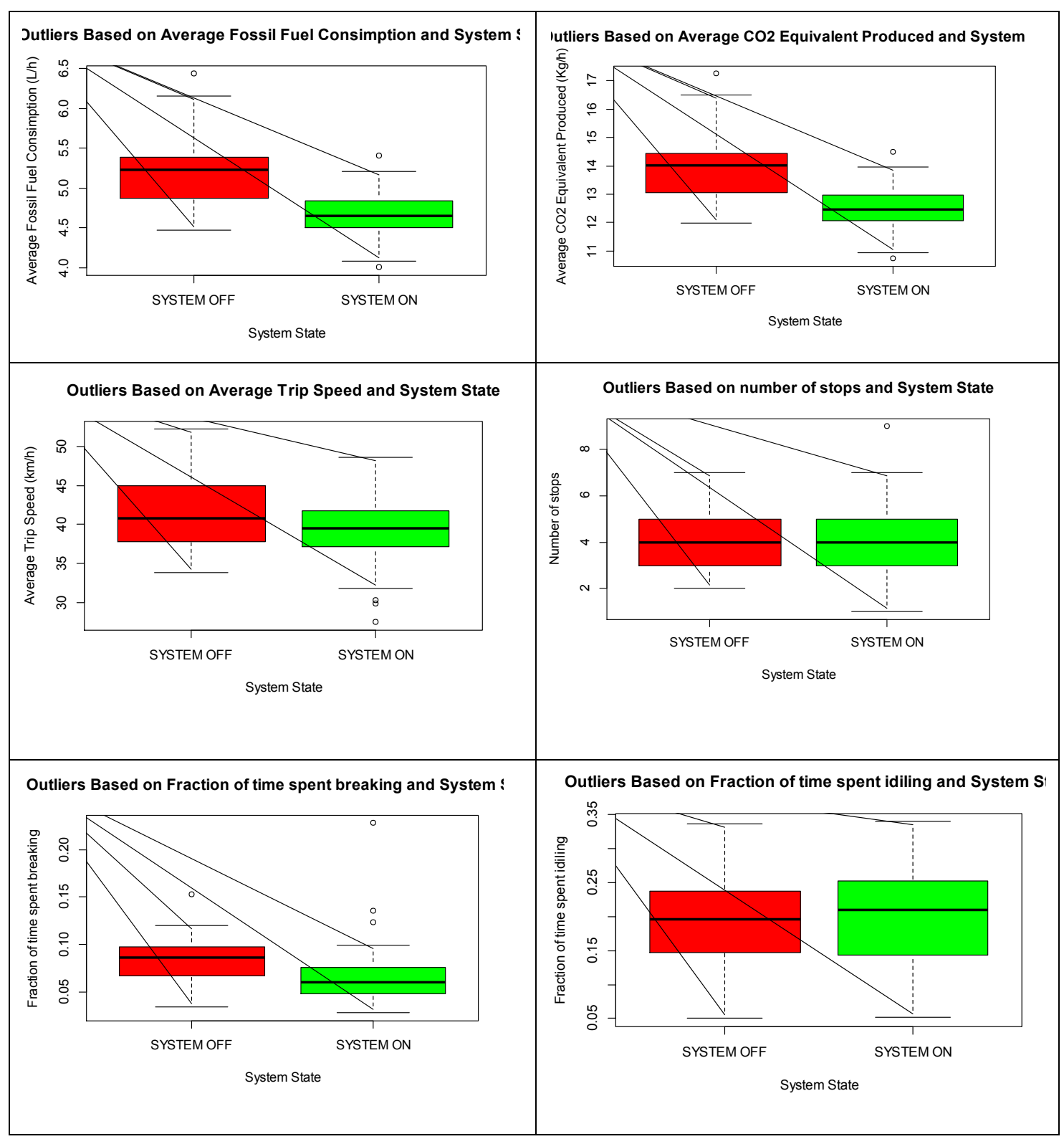

Figure 47: Trip outlier representation through boxplots 


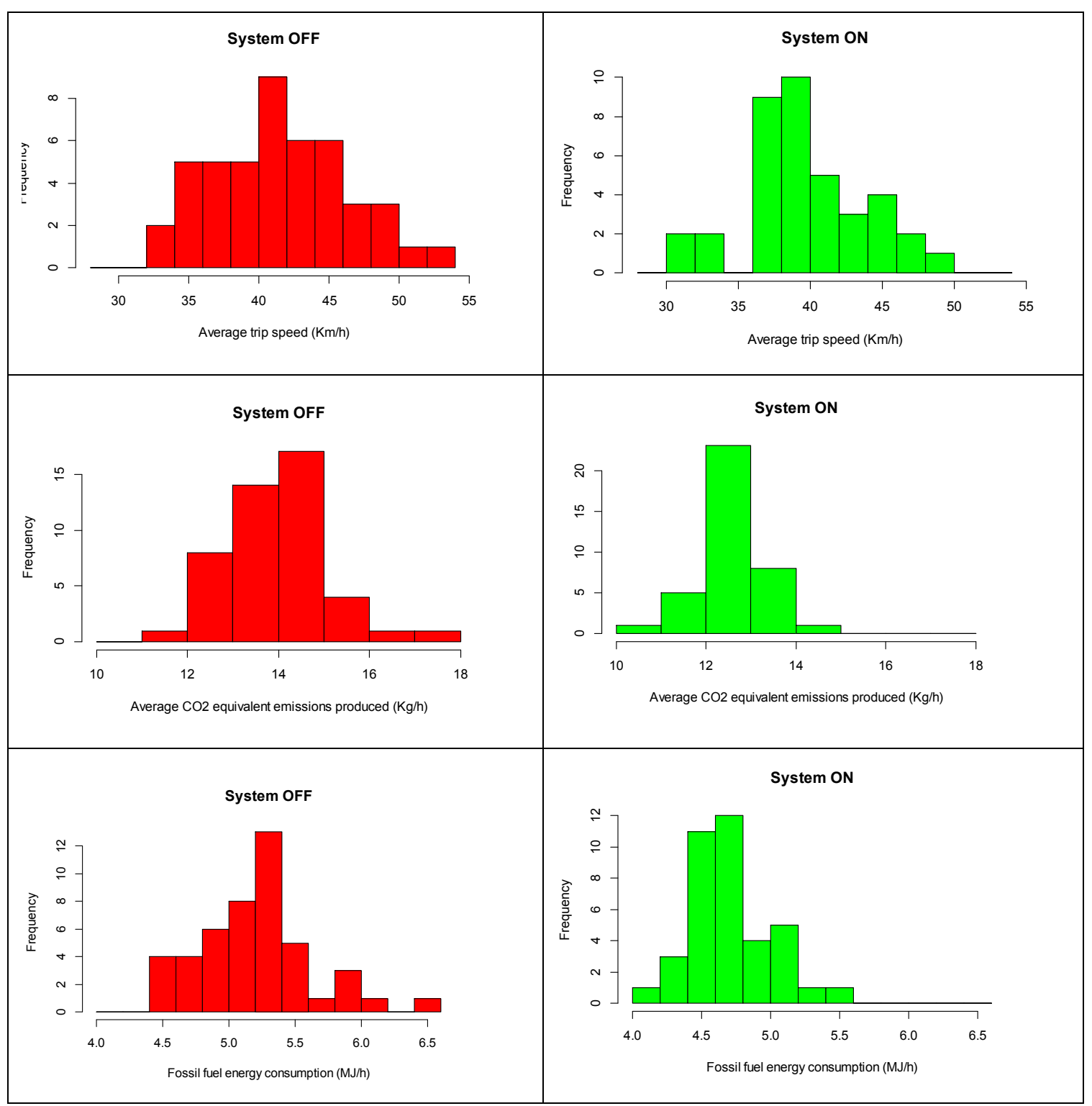

Figure 48: Frequency distributions of core variables for uninformed and informed driver phases 


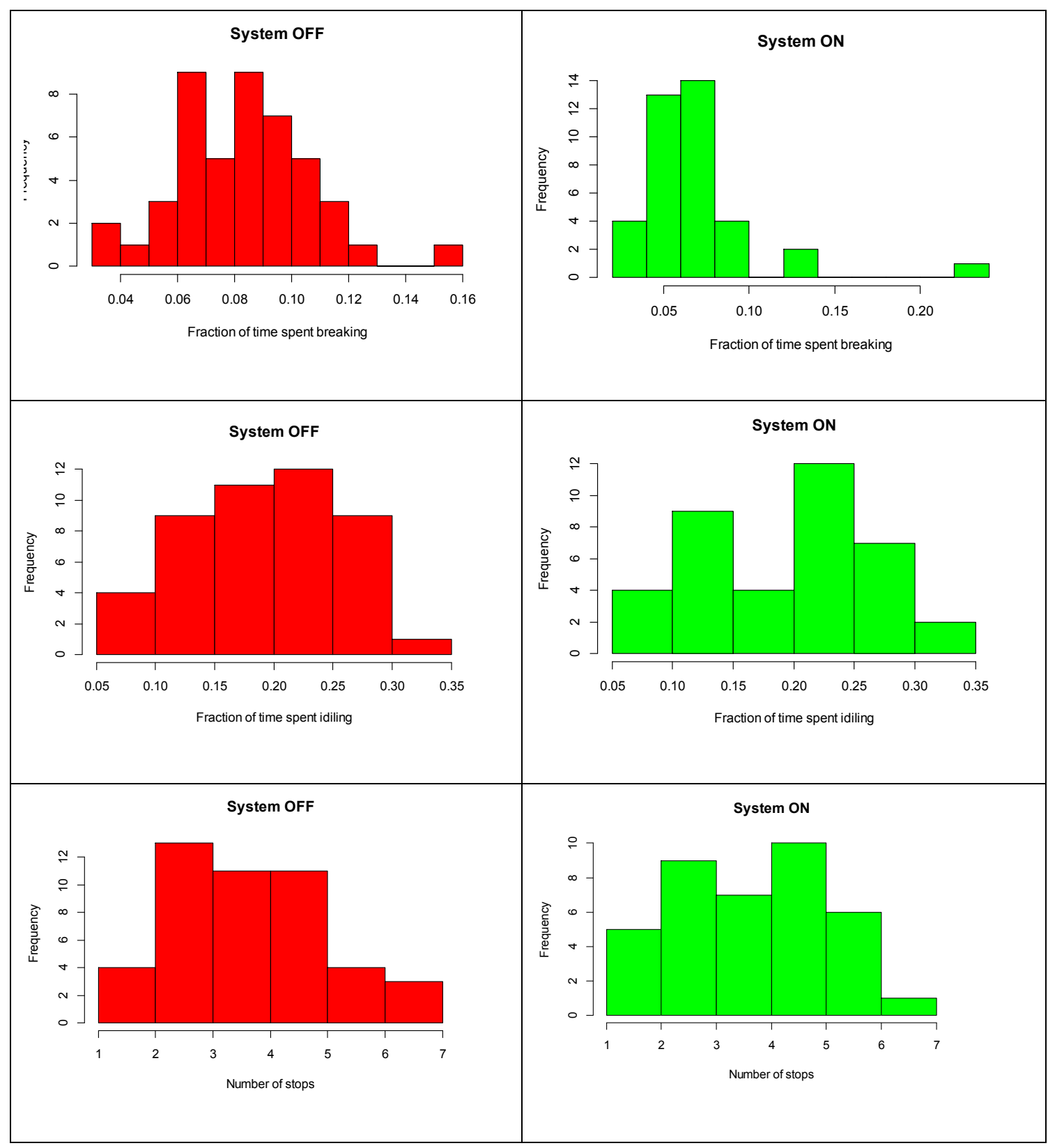

Figure 49: Frequency distributions of secondary variables for uninformed and informed driver phases 